\title{
MACARONESIAN BOTANY AND THE ENDEAVOUR VOYAGE: THE COLLECTIONS AND RECORDS OF JOSEPH BANKS AND DANIEL SOLANDER FROM MADEIRA
}

\author{
Arnoldo Santos-Guerra*, Mark A. Carine** \\ $\&$ Javier Francisco-Ortega***
}

\begin{abstract}
The efforts of Joseph Banks and Daniel Solander to document the vascular plant, bryophyte, fungal, algal, and lichen flora of Madeira during the first circumnavigation of James Cook on Her Majesty's Bark Endeavour (1768-1771) are documented. Banks's journal (at the State Library of New South Wales, Australia) provides accounts pertinent to the species observed in this visit and also includes a list of 330 entries that were recorded during their stay in this Portuguese island. Where possible, the species documented in this list were matched with corresponding herbarium collections held in the herbarium of the Natural History Museum, London, where the herbarium of Joseph Banks is now housed. Comparisons were made with two documents also housed in this Museum, namely: Solander's unpublished flora of Madeira (Primitiae Florae Maderensis, sive catalogus Plantarum in Insula Madera) and an inventory of specimens that were collected and stored inside drying books during the expedition.
\end{abstract}

KEYwords: botanical history, atlantic islands, plant taxonomy, botanical exploration, enlightenment.

\author{
BOTÁNICA MACARONÉSICA Y EL VIAJE DEL ENDEAVOUR: LAS COLECCIONES \\ Y OBSERVACIONES DE JOSEPH BANKS Y DANIEL SOLANDER DE MADEIRA
}

\section{RESUMEN}

Se examinaron, en el herbario del Museo de Historia Natural de Londres, las recolecciones de plantas vasculares, briófitos, hongos, algas y líquenes realizadas por Joseph Banks y Daniel Solander en Madeira durante el primer viaje de James Cook alrededor del mundo (1768-1771). El diario de Banks (en la Biblioteca Estatal de Nueva Gales del Sur, Australia) aporta detalles sobre las especies observadas en esta expedición y también incluye un registro de 330 entradas con las especies que se observaron durante su estancia en dicha isla portuguesa. Las especies de esta lista se estudiaron y cotejaron, cuando fue posible, con los ejemplares correspondientes del herbario del Museo de Historia Natural de Londres, donde se encuentra el herbario de Joseph Banks. Se hicieron comparaciones de este registro con datos de dos documentos que también se encuentran en este museo, a saber: la flora inédita que Solander preparó para Madeira (Primitiae Florae Maderensis, sive catalogus Plantarum en Insula Madera) y el inventario hecho por Banks y Solander de los especímenes recolectados durante la expedición que fueron preservados dentro de los libros que se usaron para secar plantas durante el viaje.

Palabras Clave: historia de la botánica, islas atlánticas, taxonomía vegetal, exploración botánica, la ilustración.

DOI: https://doi.org/10.25145/j.SI.2020.03.11

Revista Scientia Insularum, 3; diciembre 2020, pp. 165-220; ISSN: e-2659-6644 


\section{INTRODUCTION}

The three voyages of James Cook (1768-1779) were among the most important $18^{\text {th }}$ century expeditions of discovery. Collectively, his trips were the first to visit all of the Macaronesian archipelagos with the exception of the Selvagen Islands (Francisco-Ortega et al. 2015). The first voyage called at Madeira between September 12 and 18, 1768. Two of the most important figures in the history of botany, Joseph (later Sir Joseph) Banks (1743-1820) from Britain and Daniel Solander (1733-1782) from Sweden were onboard. As part of this issue of Scientia Insularum devoted to the FloraMac2018 international meeting, Prof. Jordan Goodman has focused on the main historical aspects of the visit to Madeira made by these two well-known botanists (Goodman 2020); our contribution will provide a review of the plant collections and records that they made on this island. In a subsequent study we will review the influence and impact that the contributions of Banks and Solander had on botanists such as Christen Smith (1785-1816), Leopold Von Buch (1774-1853) or Richard T. Lowe (1802-1874) who studied the Macaronesian flora, particularly in the $19^{\text {th }}$ century (Santos-Guerra in prep.).

Sydney Parkinson (1710?-1771) also joined the expedition as the illustrator responsible for botany and natural history more generally with his fellow Scot Alexander Buchan (d. 1769) responsible for landscapes and figures. Both died during the voyage. On Madeira, Parkinson made drawings of Madeiran plants, 16 of which were finished as watercolors (Figure 1). The reader can find further details of these illustrations in Francisco-Ortega et al. (2015). Eleven of Parkinson's watercolors from Madeira resulted in engravings that were eventually published in the $20^{\text {th }}$ century as part of the work known as Banks's Florilegium (Banks et al. 1985), long after they were first painted.

The herbarium collections made by Banks and Solander in Madeira are among the earliest from Macaronesia but were preceded by others made by pioneer plant collectors such as Scottish surgeon James Cuninghame (c. 1655-1709) in La Palma in 1678-1679 (Santos-Guerra et al. 2012); and the English physician and collector Sir Hans Sloane (1660-1753) in Madeira in 1687 (Menezes de Sequeira et al. 2010). Other early naturalists who contributed to the study of the Macaronesian flora included Louis Feuilleé (1660-1732), who in 1724, during his second visit to the Canaries (La Gomera, El Hierro, La Palma, and Tenerife), made the first known drawings of Macaronesian plants in their habitats (Puig-Samper and Pelayo 1997).

In this paper we present a study based on the manuscript that includes the list of Madeiran plants that Banks recorded in his two-volume expedition jour-

* Calle Guaidil 16, Canary Islands, Spain. Corresponding author: asantos0511@gmail.com.

** Department of Life Sciences, Natural History Museum, London, United Kingdom.

*** International Center for Tropical Botany, Kimberly Green Latin American and Caribbean Center, Cuban Research Institute, Department of Biological Sciences, Florida International University, Miami, Florida, USA. 


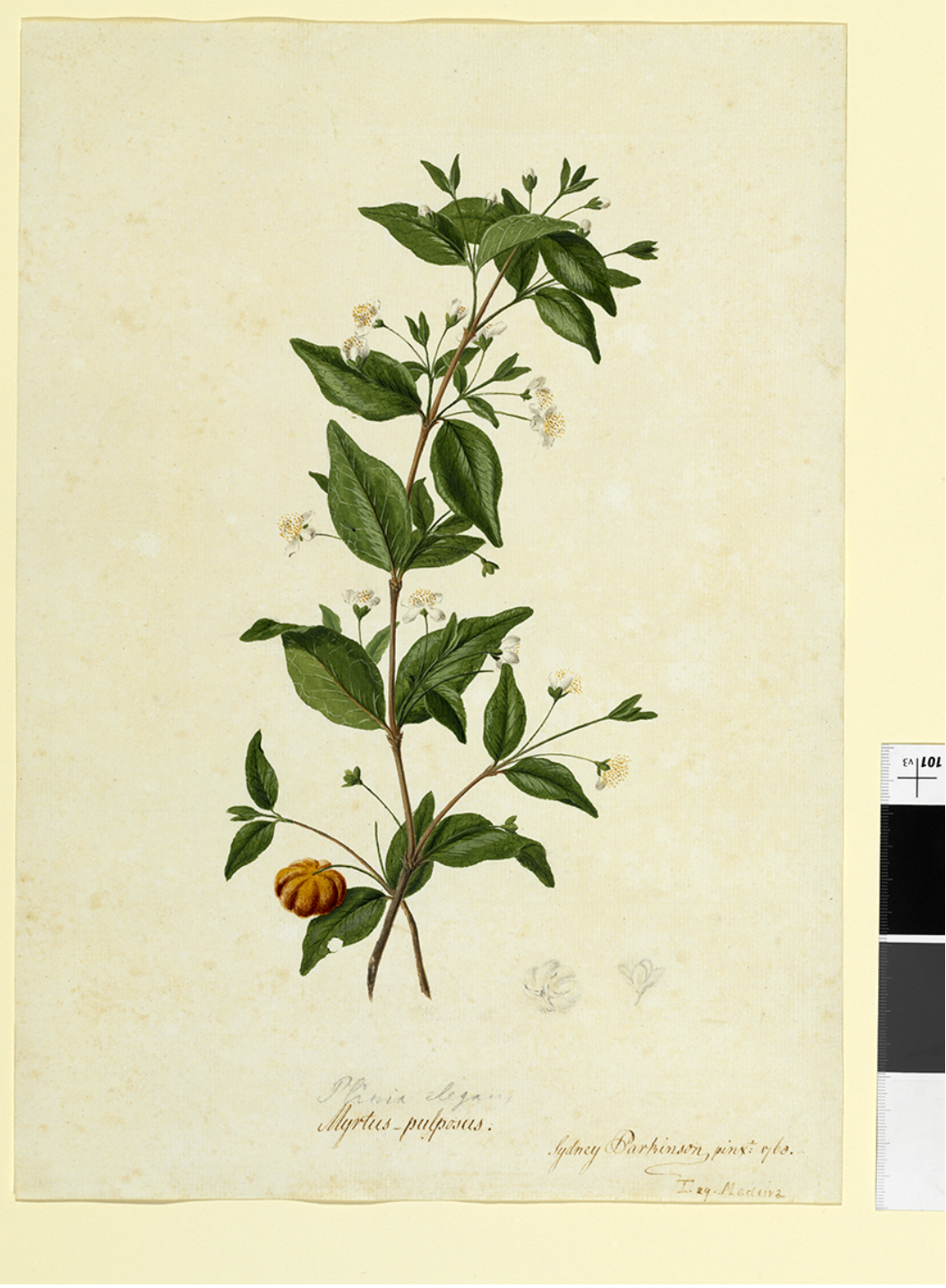

Figure 1. Watercolor of Eugenia uniflora made by Sydney Parkinson in 1768, based of material recorded in Madeira during the Endeavour voyage. Image copyright of the Natural History Museum of London.

nal housed at the State Library of New South Wales, Australia [available online in https://www.sl.nsw.gov.au/banks/section-02/series-03/03-01-volume-1-josephbanks-endeavour; Figures 2-3]. The list is part of Volume 1 of the journal and is 
tagust

25. After traving warte? in this plece ten 2ays; the thip, a vadry thiry belorping to me, heing all thet

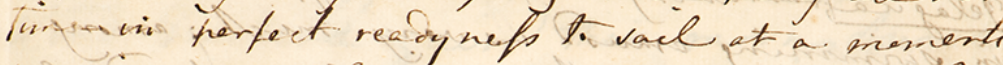

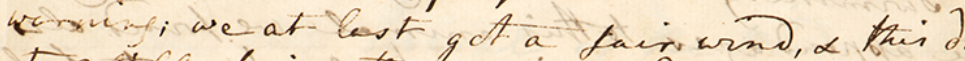

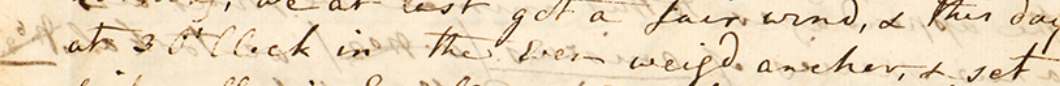

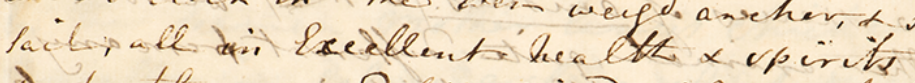

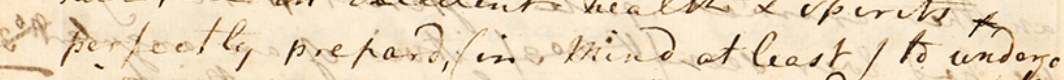

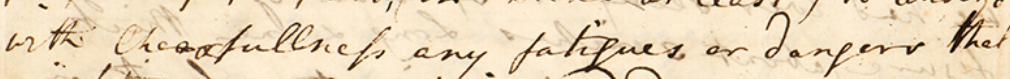

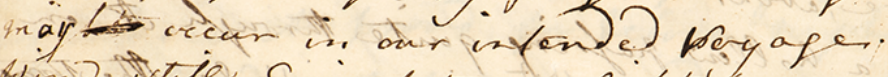

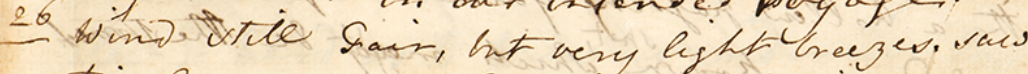
this bocn a vhoul of those ofish whirt are hartioubarly bell? Porkoisen by the seamen,

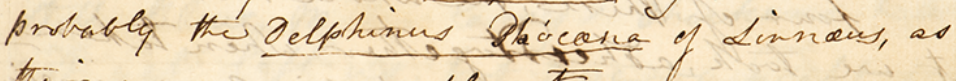
thar nowes are very blunt.

2) Wint fair o a fin Breese; trion the stip to the butia heary vilex, of Probably the indert w enld nft topeit hes to be any ther hou he milt, so are obley to ret 2 oum with thi Ancinvenieses as a pefeefory cansequence of hir trme, wish is muth more calculatet for viviges, than for owiling.

28 bitle wint to bay, in sme vea water whech

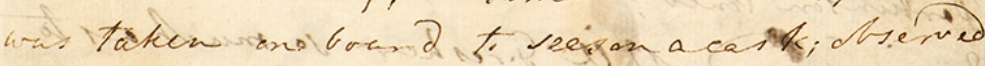
very momite sea snsect whith gryolinar

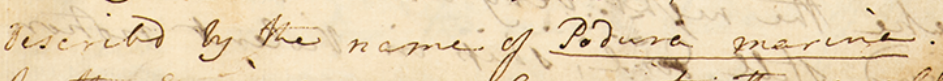
In the ivening very calm, with the vmael

Figure 2. First page of the journal of Joseph Banks, written during the first circumnavigation voyage of James Cook. Image copyright of the State Library of New South Wales, Australia.

inserted after folio 33. It comprises 13 numbered folios and has a total of 330 plant entries. Banks's journal, including the list of specimens from Madeira, was transcribed by Beaglehole (1962b: 281-289); however, the transcription of the list inclu- 


\section{Planta of Mlladia}

N.B. The mark of a Hark in the maingin ryaspies that the plaint io martid is net wisld but cultivates a crofst that forwant of fructs -icaticin the fieant conto not ecrtainly be dotermi? Monandria

Canna on dice Limn. Bananeura Grava. Centeria hereta * Amomumzing ber Lum.

Callitriche verna Limn.

Diandria

* Nyctanthes sambar Lim. Glore poleno * Tas minum officinales Limi. *

Tolea zurcpoa Lmm.

Veronica anagallis $\nabla$ Lem.

Verkena officinalis Lim.

* Ros marinus offrunali Linn

* Salvia officinalir Linn.

Triandria

Gladiolus communis Limn. Atho brabo

Iris

Cyperus ritiendus Lim.

-. - filavescens dim

Sevrnus setaceus Lim.

* Succharum officinarum Linn

Phalaris canariensis Limn.

..... oblengata infl.

Panium funceum. Dijitar Grama

Figure 3. First page of list of plants recorded in Madeira found in the journal of Joseph Banks. Image copyright of the State Library of New South Wales, Australia.

ded some errors. In our contribution we provide a literal transcription of this part of Banks's journal (see full interpreted list further below). 
One of our main aims in this paper is to provide an updated taxonomic identification of the vascular and non-vascular plants and lichens that Banks listed for Madeira in this manuscript. We matched these entries with the herbarium collections that Banks and Solander made in Madeira housed in the Natural History Museum, London (BM). As a working taxonomy we have followed Press and Short (1994), Jardim and Menezes de Sequeira (2008), and Menezes de Sequeira et al. (2012).

\section{BOTANICAL RECORDS IN BANKS'S JOURNAL}

The list of Madeiran plants found in Banks's journal comprises 330 entries (324 taxa) that include 303 spermatophytes, twelve ferns, two mosses, two liverworts, one alga, and four lichens (see full interpreted list further below). All of the identified taxa have a single entry in this list with the exception of six that have two entries each, namely: Convolvulus althaeoides (entries 65, 68), Cynoglossum creticum (entries 61, 62), Malva sylvestris (entries 203, 204), Ramalina calicaris (entries 321, 325), Olea maderensis (entries 7, 54), and Salix x rubens (entries 287, 288). Approximately half of the entries recorded in this list correspond to cultivated (72 entries) or introduced taxa ( 67 entries). Of the remaining entries, 18 are Macaronesian endemics, 19 are Madeiran endemics, and 153 are non-endemic natives.

For 94 of the entries (93 taxa), local Portuguese common names are indicated (in many cases abbreviated as "Lus."). The abbreviations "Mscr." or "Mss." were used to refer to putative new taxa, 37 in total, recognized by Banks and Solander. Since they were used only in a manuscript, they are unpublished designations although many were subsequently published by other botanists. Banks's manuscript also states (as "fig. pict.") those taxa which were illustrated by Sydney Parkinson. Two symbols were used to further identify the recorded material: asterisks $\left({ }^{*}\right)$ refer to those species that Banks and Solander regarded as cultivated or introduced (although some entries for cultivated plants are not marked) and plus symbols $(+)$ denote entries for which they were uncertain of their taxonomic identification.

During their short stay in Madeira, Banks and Solander only collected "in the neighborhood of the town [Funchal], never going above three miles from it" (September 13 record in Banks's journal). However, they were able to make an extremely rich herbarium collection including species such as Juniperus oxycedrus $(=J$. cedrus subsp. maderensis), Taxus baccata and Vaccinium padifolium, all of which are from inland parts of the island and do not occur naturally around Funchal. It is likely that they received specimens from collaborators during this visit. Thomas Heberden (1703-1769), a British physician and botanist who lived and died in Madeira (Heberden 1990) was instrumental in the discovery of botanical novelties by Banks and Solander in Madeira. Regarding the interactions that Banks and Solander had with Heberden during their stay in Madeira, from Rio de Janeiro Solander wrote a letter to the British naturalist John Ellis (c. 1710-1776) on 1 December 1768 (Beaglehole 1962b: 309-310) that stated: 
If any of your friends go to Madeira, advise them to get recommendations to Dr Heberden; he has more influence there than the governor. He is just such a philosopher as my friend, and very communicative. His many instruments, mathematical and optical, have procured him the name of il Doctore Docto.

Heberden had earlier lived in the Canary Islands (Gran Canaria and Tenerife) between the 1730s and 1747. He wrote one article about Mount Teide, Tenerife (Heberden 1752) but in 1740 he faced the Inquisition and was sent to jail. Eventually, he left the Canaries for Madeira (González Lemus 2003, 2012). In Madeira, it is likely that some of the material that Banks and Solander collected came from the gardens and plausibly the herbarium collections of Heberden himself. Interestingly, Heberden wrote a manuscript on the trees growing in Madeira, a copy of which was given to Banks as a gift "together with such specimens as he had in his possession" (entry for September 17 in Banks's journal). It appears that this manuscript by Heberden has not survived as the only publications that he wrote on Madeira concerned the climate and public health of the island as well as astronomical and geological observations and an account on an earthquake that happened in 1761 (Heberden 1990).

Banks and Solander informally named a new genus "Heberdenia" in Heberden's honor. Later, this taxon was effectively published by De Candolle (1841: 79) making reference to Banks's records from Madeira (accepted name Heberdenia Banks ex A.DC.). There has been some confusion on the correct species name of this Macaronesian endemic [Heberdenia excelsa (Aiton) Banks ex Roem. \& Schult. vs. H. bahamensis (Gaertn.) Sprague]. Treatments targeting this species indicate bahamensis is the correct species epithet for this taxon (De Wit 1957; Ståhl 1996), and this taxonomic arrangement is followed by us.

All of the species in Banks's list were identified with scientific names except four for which specimens without reproductive parts were collected: "Marmulano" (Sideroxylon mirmulans R.Br.), "Faya" (Morella faya (Aiton) Wilbur), "Pao branco" (Picconia excelsa (Aiton) DC.) and "Salvia major..." (Teucrium betonicifolium Jacq). Banks placed the "Marmulano" in the Linnean class Hexandria. Later, Francis Masson (1741-1805), first plant collector of the Royal Botanic Gardens, Kew, who botanized extensively in Madeira, Tenerife, and the Azores (São Miguel, Faial, and also possibly São Jorge) between 1776 and 1779 (Francisco-Ortega et al. 2008), found this species again. In a letter he sent to Banks, dated $4^{\text {th }}$ April 1777, Masson indicated that it belonged to the genus Sideroxylon L. (Lobin et al. 2005; Santos Guerra $e t a l$. in preparation). This interesting tree species was later described by Robert Brown (in Buch, 1825: 193) as S. mirmulans.

"Pao branco" the second species that remained unidentified in Banks's list was subsequently described in Hortus Kewensis (Aiton 1789a) as Olea excelsa Aiton, also based on Masson's collection. Aiton (1789b) used material collected by Masson to describe, the third of these "enigmatic species", the "Faya" that was named Myrica faya Aiton. The last of the species that Banks could not place taxonomically, was identified on Banks's list using a Salvia polynomial name published by Sloane (1707). Francisco-Ortega et al. (in this ussue) provided a review on the taxonomic history of this taxon indicating that Teucrium betonicifolium Jacq. is its correct name. 


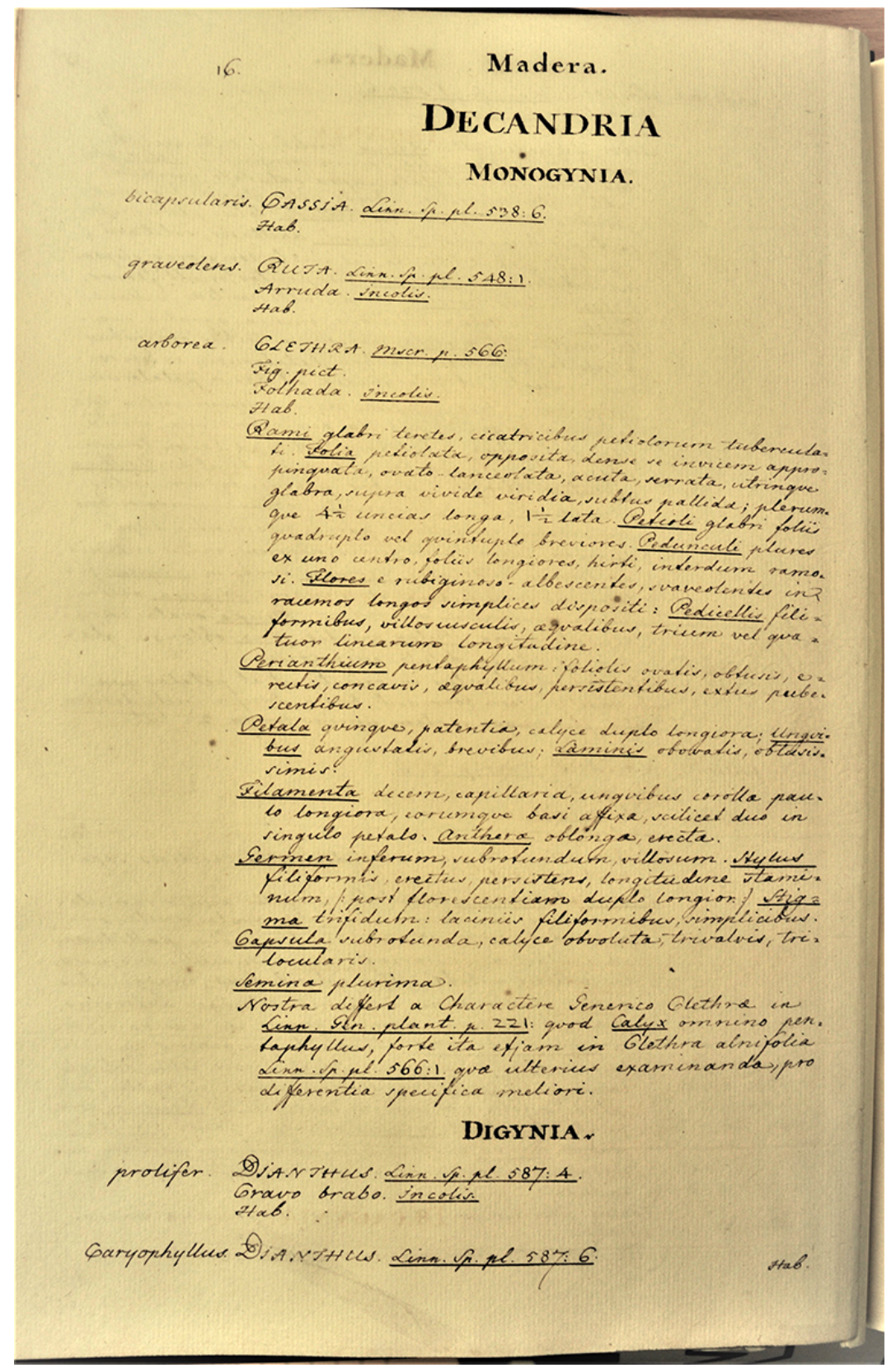

Figure 4. Page 16 of the Primitiae Florae Maderensis, sive catalogus Plantarum in Insula Madera, a work that was prepared by Daniel Solander and remained unpublished. From top to bottom: Senna bicapsularis, Ruta graveolens, Clethra arborea (including the full description of this Madeiran endemic), Petrorhagia prolifera and Dianthus caryophyllus. Image copyright of the Natural History Museum of London. 


\section{PRIMITIAE FLORAE MADERENSIS, SIVE CATALOGUS PLANTARUM IN INSULA MADERA AND EARLY IMPACT OF THE COLLECTIONS}

As with other regions visited during the Endeavour voyage, a manuscript flora was prepared for Madeira by Solander (Primitiae Florae Maderensis, sive catalogus Plantarum in Insula Madera), but it remained unpublished (Figure 4). According to annotations found in this manuscript, this copy was prepared by Sigismund Bacstrom (c.1750-1805) from Germany, who was employed as secretary to Joseph Banks between 1772 and 1775 (Cole 1980). The manuscript comprises the first 41 pages of a manuscript volume entitled "Floras of the Countries visited during Capt. James Cook's first Voyage" (Botany Manuscripts MSS BANKS COLL SOL; barcode 338012). Whilst unpublished, the Madeiran findings of Banks and Solander were relevant for later researchers. For example, in the late $18^{\text {th }}$ century, L'Héritier (1788) studied material collected by Banks and Solander for his description of Bystropogon punctatus. Robert Brown (1773-1858), Banks's librarian and later the first keeper of the Banksian (later Botanical) Department of the British Museum, based his first comprehensive list for the Madeiran flora mostly on Masson's collections housed at the BM but the collections made by Banks and Solander were also one of the obvious sources for his study. The actual date when Brown prepared this list is unknown but this floristic account remained unpublished until the works of Buch (1825: 189-199) and Britten (1904). It is noteworthy that Brown himself visited Madeira in 1801 in route to Australia on board HMS Investigator (Moore 2001).

Solander's Primitiae Florae Maderensis, sive catalogus Plantarum in Insula Madera included 351 entries for 345 taxa. Twenty of these entries refer to polynomial names published by Plukenet and Sloane (revised by Francisco-Ortega et al. 1994 and Menezes de Sequeira et al. 2010). We infer that the remaining entries (331 entries, 325 taxa) are for plants that were recorded or collected in Madeira by Banks and Solander as they match those found in Banks's journal; however, four of these 325 taxa [Boletus sp. (Boletaceae), Bystropogon punctatus (as Menthastrum Mentha maderensis in Solander's list), Lichen subfuscus L. (Lecanoraceae), and Lotus corniculatus L. (= L. lancerottensis Webb \& Berthel.) (Fabaceae)] were not listed in Banks's journal. Lichen subfuscus [accepted name Lecanora subfusca (L.) Ach. (LIAS 19952020)] and the fungal genus Boletus s.l. both occur in Madeira (Carvalho et al. 2008, Melo and Cardoso 2008) but no herbarium material collected by Banks and Solander was seen during the course of this study. Two of the taxa recorded in Banks's journal (Campanula erinus and the garden plant Gardenia jasminoides) are not found in Primitiae Florae Maderensis.

In this pioneering flora, Solander wrote descriptions for 15 Madeiran/Macaronesian endemics that later were validly published by other taxonomists, namely: "Alypum longifolium" (Globularia salicina); "Clethra arborea" (C. arborea, Figure 4), "Crepis tenuifolia" (Tolpis succulenta, C. succulenta); "Heberdenia excelsa" (H. bahamensis, Anguillaria bahamensis Gaertn.), "Hypericum evectum" (H. grandifolium), "Hypericum glandulosum" (H. glandulosum), "Ilex azevinho" (I. canariensis), "Ilex perado" (I. perado subsp. perado), "Laurus nitida" (Apollonias barbujana, L. barbu- 
jana Cav.), "Lavandula pinnata" (L. pinnata), "Laurus foetens" (Ocotea foetens, L. foetens), "Lotus glaucus" (L. glaucus), "Rubus pedatus" (R. serrae), "Smilax latifolia" (S. pendulina), and "Vaccinium elevatum" (V. padifolium).

\section{THE HERBARIUM RECORD}

Shortly before his death in 1820, Banks bequeathed his herbarium to his librarian Robert Brown on the condition that it would become the property of the British Museum on Brown's death - unless Brown chose to transfer it earlier. Negotiations for its transfer began soon after Banks's death. Favourable terms were agreed and in 1827 the herbarium was transferred with Brown appointed Keeper of the newly formed Banksian Department as part of the agreement. The herbarium was transferred to South Kensington, London in 1881 where it remains today and forms the one of the foundation collections of the Natural History Museum herbarium (BM).

We have found matching herbarium specimens in three different collections of the Natural History Museum herbarium (Figures 5-7), namely (1) the General Herbarium, (2) a bound volume entitled "Plants of Cook's First Voyage 1768-1771" held in the Historical Collections Room, and (3) inside a drying book ('Madeira III') made up of loosely bound uncut pages from Adison's Notes upon the twelve books of Paradise Lost, published in 1719. This was one of seven bundles of plants resulting from Banks and Solander's collecting activities in Madeira. It is the only one to survive intact and with some specimens still contained within it.

During the course of Cook's voyage specimens were stored inside these drying books [see Groves (1962) for a full account regarding the protocols followed by Banks and Solander in preparing and recording the extensive collections of specimens that were made during this Endeavour voyage]. After Banks and Solander's return to Britain this material was removed and subsequently mounted on sheets (material now in the General Herbarium) and/or inside the volumes of "Plants of Cook's First Voyage 1768-1771" (Edwards 1978), located in the historical collections room at BM.

In total, there are 210 collections for 206 taxa in the Natural History Museum herbarium. Sixteen of the taxa are Madeiran endemics, 18 are Macaronesian endemics, 100 are non-endemic natives, 60 are introduced, and 12 are cultivated. These specimens represent ten fern and 196 spermatophyte taxa. As the list of material recorded in Banks's journal has 163 introduced taxa, these data show that $44 \%$ of the records for introduced taxa lack matching specimens in the herbarium. This suggests that Banks and Solander mostly focused their collecting efforts on plants belonging to the native flora, including the endemics.

One-hundred and ten of these collections (108 taxa) are found only in the General Herbarium, 25 of them (25 taxa) are found only in the bound volume of Plants of Cook's First Voyage and 75 (75 taxa) are shared by these two herbarium holdings. Unfortunately it was not possible to fully assess the contents of the drying book (Madeira III) because of its fragility. However, it is clear that the drying book has relatively few specimens and it is unlikely that there are any that are not found in the General Herbarium or the Plants of Cook First Voyage bound volume. 


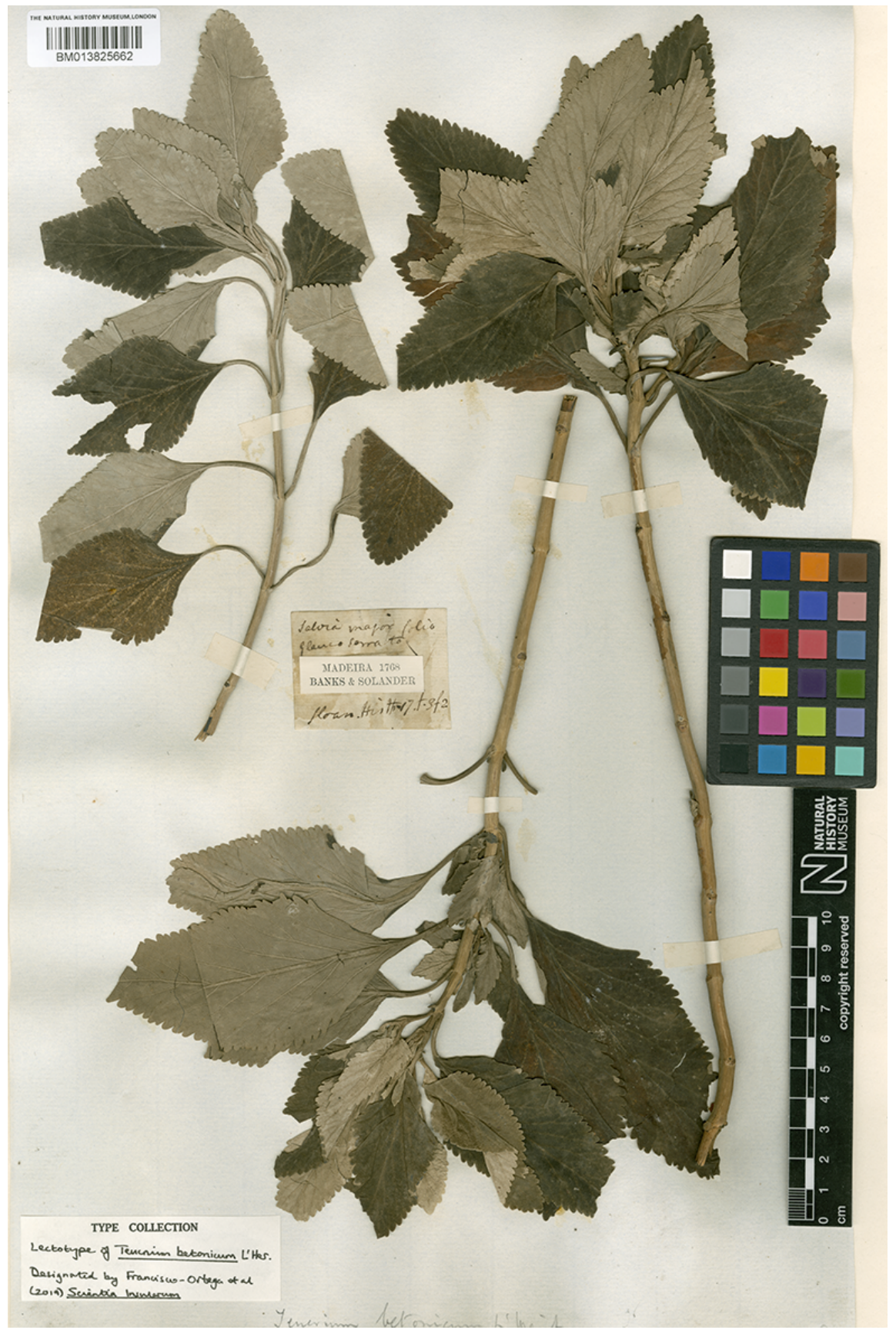

Figure 5. Herbarium specimen of Teucrium betonicifolium collected by Banks and Solander in Madeira during the Endeavour voyage. This is the lectotype of T. betonicum L'Hér., as designated by Francisco-Ortega et al. (in this ussue). Image copyright of the Natural History Museum of London. 


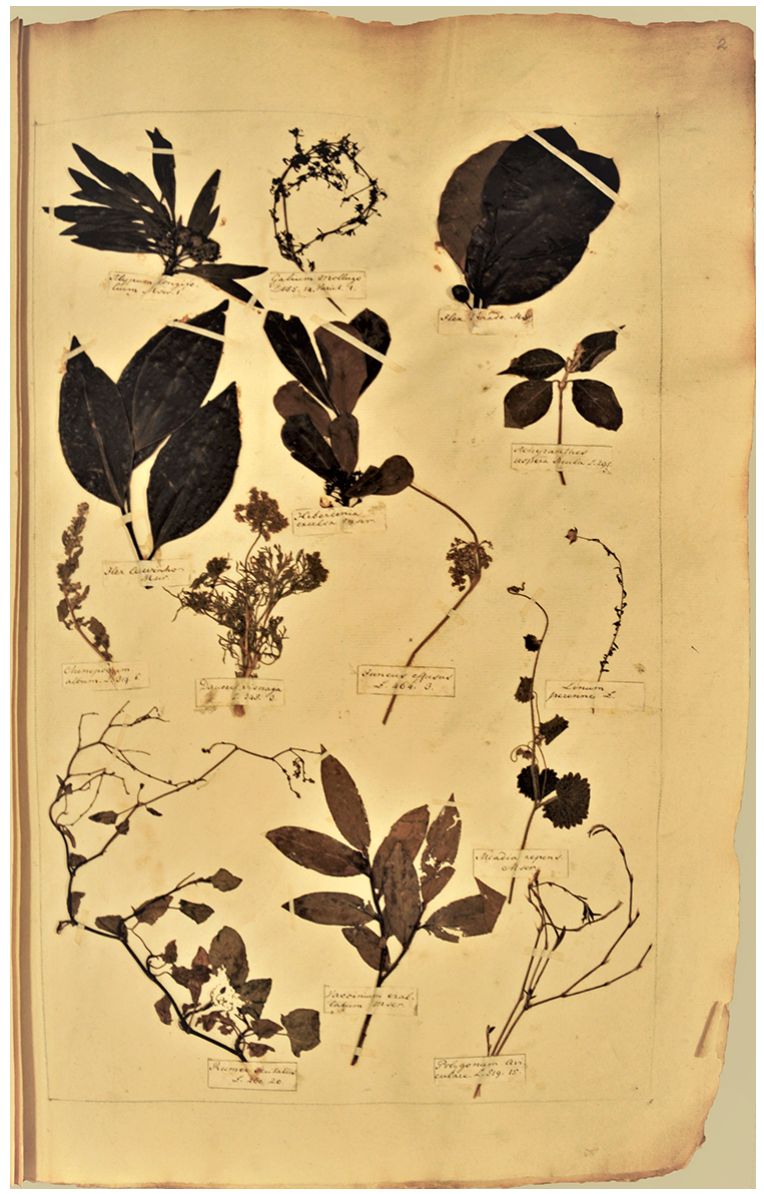

Figure 6. Sheet with specimens collected by Banks and Solander in Madeira that is part of a bound volume (folio 2) entitled Plants of Cook's First Voyage 1768-1771 held in the Historical

Collections Room of the Natural History Museum of London. Specimens are identified (left to right) as follows: Top row: Globularia salicina, Galium productum, Ilex perado perado subsp. Second row from top: Ilex canariensis, Heberdenia bahamensis, Achyranthes sicula. Third row from top: Chenopodium album, Ammi visnaga, Juncus effusus, Linum bienne. Bottom row: Rumex maderensis, Vaccinium padifolium, Sibthorpia peregrina, Polygonum aviculare. Image copyright of the Natural History Museum of London.

\section{The Drying Book Inventory}

The Natural History Museum library houses a further list of Madeiran plants that forms part of a manuscript entitled 'Catalogue of the plants collected at Madeira, Brazil, Tierra del Fuego and the Society Islands' (31pp, Botany Manus- 


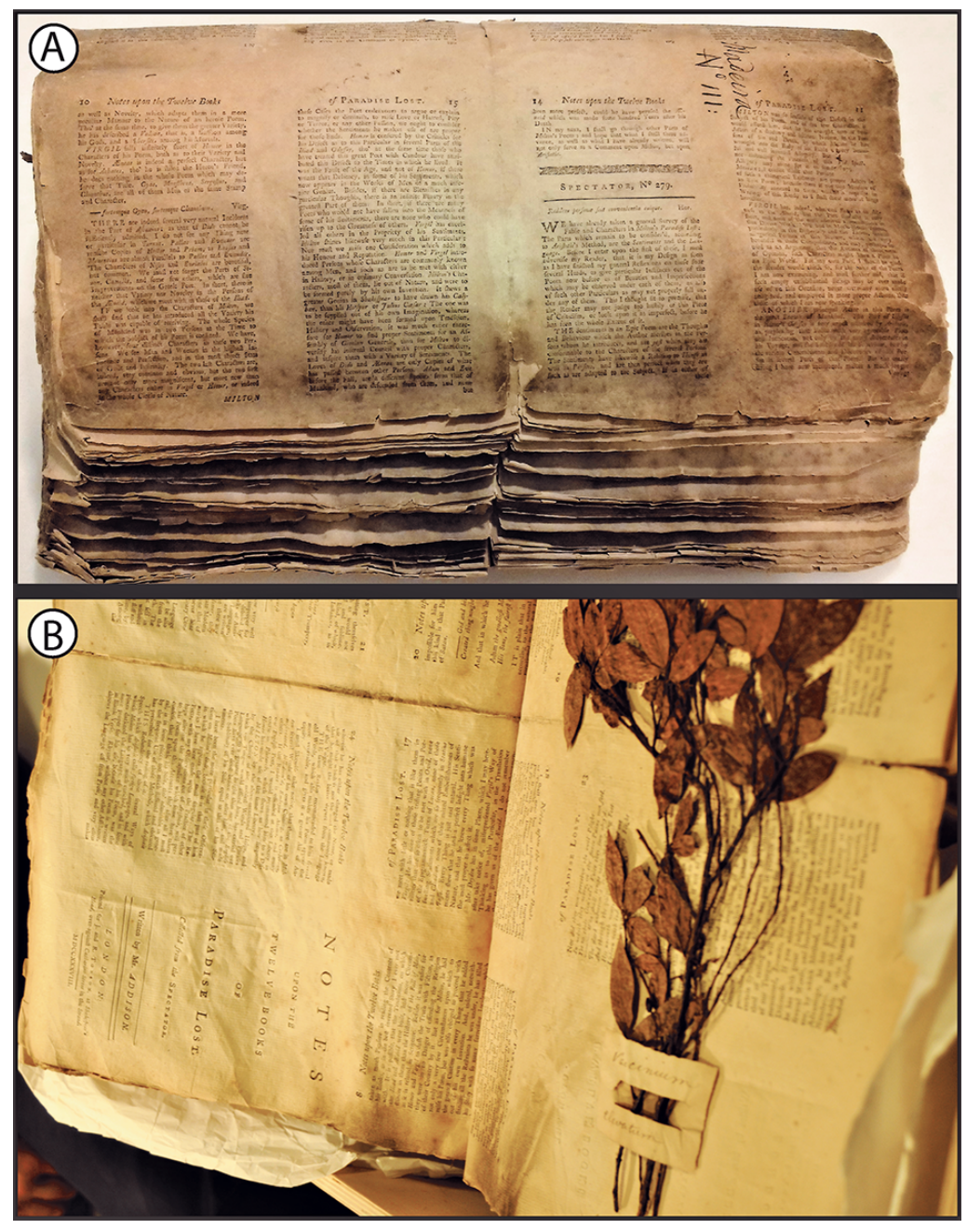

Figure 7. Drying book (Madeira III) that contains plant specimens collected in Madeira during the visit of Endeavour from 12-18 September, 1768. A: General view of the bundle that makes this particular drying book. B: Specimen of Vaccinium padifolium, notice that the bottom of folio that faces the specimen has the full title of one of the uncut pages of the book that was used to dry plants during the expedition. Image copyright of the Natural History Museum of London.

cripts MSS BANKS COLL; barcode 138798). It documents 256 entries (252 taxa) for spermatophytes (231 taxa), ferns (11 taxa), algae (one taxon), mosses (two taxa), liverworts (two taxa), and lichens ( 5 taxa) for which herbarium material was collected in Madeira (Figure 8). The last page of this manuscript erroneously indicates that it 


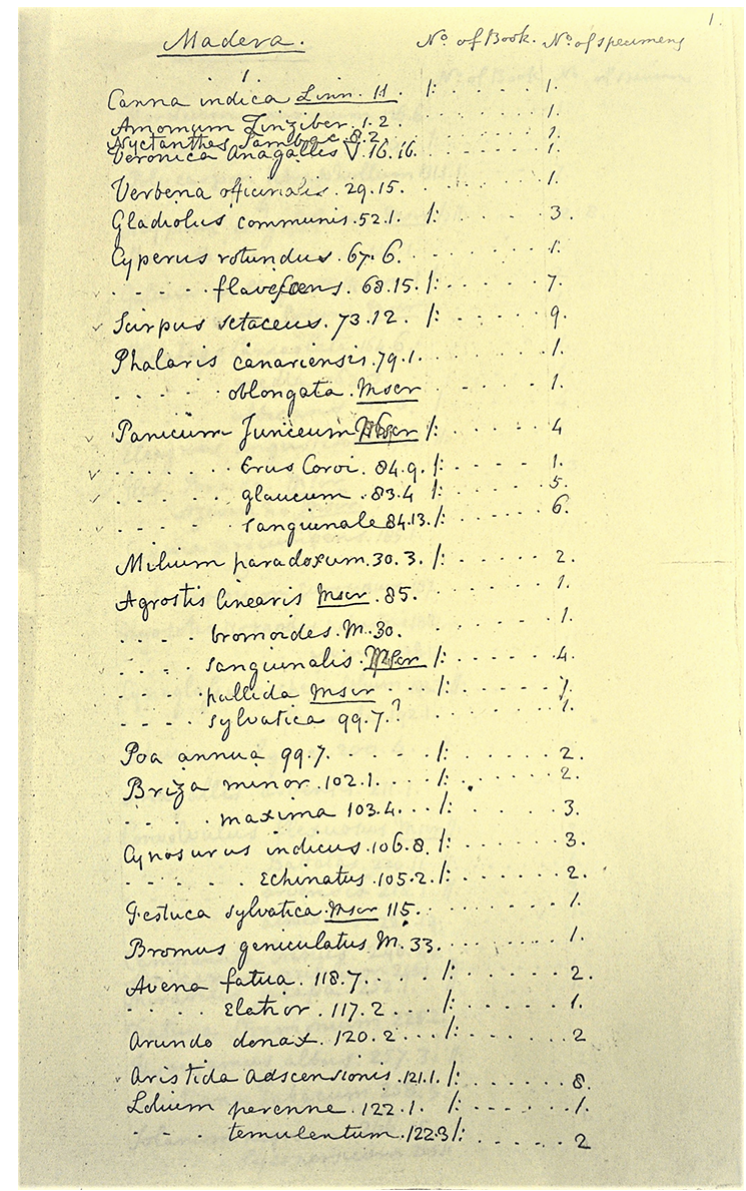

Figure 8. First folio of the eight folio list (256 entries) that has an inventory of the Madeiran collections made by Banks and Solander. This is part of the index to specimens that were stored inside drying books during Cook's voyage. Numbers after each name refer to the page and plant numbers in the second edition of Linnaeus' $(1762,1763)$ Species plantarum. "No of Book" refers to the drying book number where the specimen was stored. The number of specimens made per collection is also indicated. The 34 plant entries listed in this page match entries found in the first two pages of the list of material included in Banks's journal.

Image copyright of the Natural History Museum of London.

comprises 253 entries instead of 256. Based on notes written on this manuscript by Eric W. Groves (1923-2012) in 1982, it appears that this list was written by Banks. All of the entries with the exception of Lotus corniculatus (= L. lancerottensis), Lichen subfuscus (see above discussion regarding the status of these two species in Banks's collections), and Bystropogon punctatus are also recorded in the list of plants 
in Banks's journal. The manuscript is an inventory of the material that was placed inside the drying books during the first part of the Endeavour voyage. It provides details of the number of duplicates made for each collection and the drying book in which each collection was stored (numbered I through vir for Madeira). The five species with the highest number of duplicates were Apollonias barbujana (14 duplicates), Heberdenia bahamensis (20 duplicates), Ilex perado (13 duplicates), Lythrum hyssopifolia (14 duplicates), and Ocotea foetens (14 duplicates). Four species (Acanthus mollis, Chelidonium majus, Equisetum arvense, and Tropaeolum majus) with matching specimens in BM were not found in this manuscript but were included both in Solanders' Primitiae Florae Maderiensis and in Bank's journal list.

For $59(23 \%)$ of the 252 taxa listed in this inventory, there are no matching specimens in BM. Twenty-five of these taxa are introduced, three have been identified as Madeiran endemics (Aichryson divaricatum, Hedera maderensis, and Sideritis candicans). Among the 25 non-endemic natives that lack specimens, one is an alga, five are lichens, four are bryophytes, two are ferns, and the remaining 13 are spermatophytes. The fate of these specimens is not clear. However, we do know that when the Banksian herbarium was transferred from his house in Soho Square to the British Museum in 1827, a substantial proportion was unmounted [estimated by Brown in 1834 to comprise 1700 bundles (Murray 1904: 80)]. The missing specimens may have been lost between the point of collection and their eventual processing for the herbarium. Furthermore, bomb damage to the BM herbarium during the Second World War affected Banks's collections (Groves 1962) and could also account for the absence of some specimens.

\section{TAXONOMIC UPDATE OF THE LIST OF MADEIRAN VASCULAR PLANTS, BRYOPHYTES, LICHENS, AND ALGAE REPORTED IN BANKS'S JOURNAL}

Below we provide a transcription of the list of vascular plants, bryophytes lichens, and algae recorded in Madeira as found in Banks's journal housed in the State Library of New South Wales, Australia. Entries are presented in the same order as they were listed by Banks which followed Linnean orders. For each entry we assign sequential entry numbers to help the reader to locate each of these records. These were not in the original list. Appendix 1 provides a taxonomic index that ties the identified species with the particular entries found in Banks's list. For each entry we transcribed the name as written in Banks lists, this is followed by text inside brackets that provides the accepted scientific name. We also provide information on status using the following abbreviations: Cult. $=$ Cultivated, Intr. $=$ Naturalized non-natives, Nat. $=$ Non-endemic natives, Mad. $=$ Madeira endemics, Mac. $=$ Macaronesian endemics, Notes $=$ Additional comments. For the most part, notes concern determinations provided by taxonomic experts. Where there is no indication that the specimen was revised for the Flora of Madeira project (Press and Short 1994), that was based at BM, this is indicated. We list specimens for each entry herbarium collections at the BM (Herb.) following this code: $\mathrm{GH}=$ General herbarium of the 
Natural History Museum of London, SCV = Volume with specimens from Cook's first voyage. Concerning entries recorded in the two other documents (Other lists), the following code is followed: NPL = Banks's index for specimens that were stored inside the drying books; SOL = Solander's Primitiae Florae Maderensis.

Sources to check the current presence of these taxa in Madeira were Press and Short (1994; hereafter Flora of Madeira in "Notes"), Carvalho et al. (2008), Jardim and Menezes de Sequeira (2008), Melo and Cardoso (2008), Sérgio et al. (2008), and Menezes de Sequeira et al. (2012).

Our taxonomic identifications are based on the study of the available herbarium material collected by Banks and Solander. For those entries for which no specimens were located, taxonomic inferences were based on: (1) the scientific and common names reported in Banks and Solander's lists; (2) references to names reported in the second edition of Linnaeus's $(1762,1763)$ Species plantarum; and (3) other sources. References to Linnaeus $(1762,1763)$ are found in Solander's Primitiae Florae Maderensis, sive catalogus Plantarum in Insula Madera and in the inventory of specimens that is part of the Catalogue of the plants collected at Madeira, Brazil, Tierra del Fuego and the Society Islands. References to Linnean names and works (mostly to Species Plantarum or Mantissa) were also found on the original labels of several of the studied specimens. When our contribution was submitted, BM was shut because of the coronavirus pandemic. Therefore we could not double check herbarium specimens for entries 52 and 202; we have tentatively assigned them to Plantago major and Sida spinosa, respectively.

[Page 1]

Plants of Madeira

N.B. the mark of a start * in the margin signifies that the plant so marked is not wild but cultivated a cross + that for want of firmely =ication [identification] the plant could not certainly be determind [determinated]

MONANDRIA

=1.- Canna indica Linn. Bananeira brava. Conteira preta. [Canna indica L. (Cannaceae)]; Intr.; Herb.: GH; Other lists: NPL, SOL; Notes: Not revised for Flora of Madeira.

=2.- *Amomun Zingiber Linn. [Zingiber officinale Roscoe (Zingiberaeae);]; Cult.; Herb.: GH; Other lists: NPL, SOL; Notes: "ex Hort" on sheet back.

=3. - Callitriche verna Linn. [Callitriche stagnalis Scop. (Plantaginaceae)]; Nat.; Other lists: SOL.

DIANDRIA

$=4 .-{ }^{*}$ Nyctanthes sambac Linn. Flore pleno. [Jasminum sambac (L.) Aiton (Oleaceae)]; Cult.; Herb.: GH; Other lists: NPL, SOL; Notes: Not revised for Flora of Madeira.

=5.- *Jasminum officinale Linn. [Jasminum officinale L. (Oleaceae)]; Cult.; Other lists: SOL. 
=6.- Jasminum grandiflorum Linn. [Jasminum grandiflorum L. (Oleaceae)]; Cult.; Other lists: SOL.

=7.- +Olea europea Linn. [Olea maderensis (Lowe) Rivas Mart. \& del Arco (Oleaceae)]; Mad.; Herb.: GH; Other lists: SOL; Notes: Revised by Lowe.

=8. - Veronica anagallis $\nabla$ Linn. [Veronica anagallis-aquatica L. (Scrophulariaceae)];

Nat.; Herb.: GH; Other lists: NPL, SOL.

=9.- Verbena officinalis Linn. [Verbena officinalis L. (Verbenaceae)]; Intr.; Other lists: NPL, SOL.

=10.- ${ }^{*}$ Rosmarinus officinalis Linn. [Rosmarinus officinalis L. (Lamiaceae)]; Cult.;

Other lists: SOL.

$=11 .-{ }^{*}$ Salvia officinalis Linn. [Salvia officinalis L. (Lamiaceae)]; Cult.; Other lists: SOL.

\section{TRIANDRIA}

=12.- Gladiolus communis Linn. Alho brabo. [Gladiolus cf. italicus Mill. (Iridaceae)]; Intr.; Herb.: GH; Other lists: NPL, SOL.

$=13$.- Iris [Iris sp. (Iridaceae)]; Intr. and Cult.; Other lists: SOL.

=14.- Cyperus rotundus Linn. [Cyperus rotundus L. (Cyperaceae)]; Intr.; Other lists: NPL, SOL.

=15.- Cyperus flavescens Linn. [Pycreus flavescens (L.) Rchb. (Cyperaceae)]; Nat.; Herb.: SCV; Other lists: NPL, SOL.

=16. Scirpus setaceus Linn. [Isolepis setacea (L.) R.Br. (Cyperaceae)]; Nat.; Herb.: GH (BM000829271), SCV; Other lists: NPL, SOL.

=17.- ${ }^{*}$ Saccharum officinarum Linn. [Saccharum officinarum L. (Poaceae)]; Cult.; Other lists: SOL.

=18.- Phalaris canariensis Linn. [Phalaris canariensis L. (Poaceae)]; Nat.; Other lists: NPL, SOL.

=19.- Phalaris oblongata Mss. [Phalaris coerulescens Desf. (Poaceae)]; Nat.; Herb.: GH; Other lists: NPL, SOL; Notes: Determined by T.A. Cope, 18.V.1989. =20.- Panicum Junceum Mscr. [crossed] Grama [Panicum repens L. (Poaceae)];

Nat.; Herb.: GH, SCV; Other lists: NPL, SOL; Notes: Not revised for

Flora of Madeira.

[Page] 2

Plants of Madeira

=21.- Panicum glaucum Linn. [Pennisetum glaucum (L.) R.Br. (Poaceae)]; Intr.; Herb.: SCV; Other lists: NPL, SOL.

=22.- Panicum sanguinale Linn. [Digitaria sanguinalis (L.) Scop. (Poaceae)]; Intr.;

Herb.: GH, SCV; Other lists: NPL, SOL; Notes: Confirmed by T.A. Cope 18.V.1989, for Flora of Madeira.

Panicum glaucum Linn. [crossed]

=23-. --.--- Cruz Corvi Linn. Milhoa Lus. [Echinochloa colona (L.) Link (Poaceae)]; Intr.; Herb.: GH, SCV; Other lists: NPL, SOL; Notes: Confirmed by T.A. Cope, 18.V.1982 included in the sheet there is another specimen determinated as Echinochloa crus-galli (L). P. Beauv. 
=24.- Milium paradoxum Linn. [Oryzopsis miliacea (L.) Asch. \& Scheweinf. (Poaceae)]; Nat.; Herb.: SCV; Other lists: NPL, SOL; Notes: This species is listed as Piptatherum miliaceum (L.) Coss. subsp. miliaceum by Menezes de Sequeira et al. (2012).

=25.- Agrostis linearis Mscr. [Agrostis sp. (Poaceae)]; Other lists: NPL, SOL. =26.- -..---- bromoides Linn. [Vulpia bromoides (L.) Gray (Poaceae)]; Nat.; Herb.: GH; Other lists: NPL, SOL; Notes: "In apricis Mantissa 30" in the original label. Confirmed by T.A. Cope, 21.VII.1989.

=27.- ------- sanguinalis Mscr. [Digitaria ciliaris (Retz.) Koeler. (Poaceae)]; Intr.;

Herb.: GH, SCV; Other lists: NPL, SOL.

=28.- -..--.-- pallida Mscr. [Gastridium ventricosum (Gouan) Schinz \& Thell. (Poaceae)]; Nat.; Herb.: SCV; Other lists: NPL, SOL.

=29.- ------- sylvatica Linn. [Polypogon viridis (Gouan) Breist. (Poaceae)]; Nat.; Other lists: NPL, SOL.

=30.- Poa annua Linn. [Poa annua L. (Poaceae)]; Nat.; Herb.: GH, SCV; Other lists: NPL, SOL; Notes: Confirmed by T.A. Cope, 21.VII.1989.

=31.- Briza minor Linn. [Briza minor L. (Poaceae)]; Nat.; Herb.: GH, SCV; Other lists: NPL, SOL; Notes: Revised by T.A. Cope, 21.VII.1989, for Flora of Madeira.

=32.- -.---- maxima Linn. Xucalheira Lus. [Briza maxima L. (Poaceae)]; Nat.; Herb.: GH, SCV; Other lists: NPL, SOL; Notes: Revised by T.A. Cope, 21.VII.1989, for Flora of Madeira.

=33.- Cynosurus indicus Linn. [Eleusine indica (L.) Gaertn. (Poaceae)]; Intr.; Herb.: GH, SCV; Other lists: NPL, SOL; Notes: Confirmed by T.A. Cope, 7.XII.1988.

=34.- -.---.-- echinatus Linn. [Cynosurus echinatus L. (Poaceae)]; Nat.; Herb.: GH, SCV; Other lists: NPL, SOL; Notes: Not revised for Flora of Madeira.

=35. - Festuca sylvatica Mscr. Bromus pinnatus Linn. [Brachypodium sylvaticum (Huds.) P.Beauv. (Poaceae)]; Nat.; Herb.: GH; Other lists: NPL, SOL; Notes: Confirmed by T. A. Cope, 8.X.1988, for Flora of Madeira.

=36.- Bromus geniculatus Linn. [Bromus madritensis L. (Poaceae)]; Nat.; Herb.: GH; Other lists: NPL, SOL; Notes: Revised by T.A. Cope, 8.X.1988, for Flora of Madeira.

=37.- Avena fatua Linn. Balanco Lus. [Avena barbata Pott ex Link (Poaceae)]; Nat.; Herb.: GH; Other lists: NPL, SOL; Notes: Confirmed by T.A. Cope, 13.IV.1989, for Flora of Madeira.

=38.- -.-.--- elatior Linn. [Arrhenatherum elatius (L.) J.Presl \& C. Presl subsp. bulbosum (Willd.) Schübl. \& G. Martens (Poaceae)]; Nat.; Other lists: NPL, SOL.

=39.- ------- nodosa Linn. [Arrhenatherum elatius (L.) J.Presl \& C.Presl subsp. bulbosum (Willd.) Schübl. \& G. Martens (Poaceae)]; Nat.; Other lists: SOL. =40.- Arundo donax Linn. Canavieira Lus. [Arundo donax L. (Poaceae)]; Intr.; Other lists: NPL, SOL.

=41.- Aristida Adscentionis Linn. [Aristida adscensionis L. (Poaceae)]; Nat.; Herb.: GH, SCV; Other lists: NPL, SOL; Notes: Not revised for Flora of Madeira. 
=42.- Lolium perenne Linn. [Lolium perenne L. (Poaceae)]; Intr.; Herb.: GH; Other lists: NPL, SOL; Notes: Not revised for Flora of Madeira.

=43.- -------- temulentum Linn. [Lolium temulentum L. (Poaceae)]; Intr.; Herb.:

GH; Other lists: NPL, SOL; Notes: Not revised for Flora of Madeira.

=44. - Hordeum murinum Linn. [Hordeum murinum L. subsp. glaucum (Steudel)

Tzvelev (Poaceae)]; Nat.; Herb.: GH (BM000060788); Other lists: NPL,

SOL; Notes: Determined by T.A. Cope, 8.X.1988.

=45.- Triticum repens Linn. Forocapa Lus. [Elymus repens (L.) Gould (Poaceae)];

Intr.; Other lists: NPL, SOL.

=46.- Polycarpon tetraphyllum Linn. Saboira Lus. [Polycarpon tetraphyllum (L.) L.

(Caryophyllaceae)]; Nat.; Herb.: GH, SCV; Other lists: NPL, SOL; Notes:

Not revised for Flora of Madeira.

[Page] 3

Plants of Madeira

Tetrandria

=47.- Alypum angustifolium Fig. pict. Mscr. Malforada Lus. [Globularia salicina Lam. (Globulariaceae)]; Mac.; Herb.: GH (BM000999098, BM000999099), SCV; Other lists: NPL, SOL; Notes: Listed as "Alypum longifolium Mscr." in NPL and SOL. Not revised for Flora of Madeira.

$=48 .-$ Sherardia arvensis Linn. [Sherardia arvensis L. (Rubiaceae)]; Nat.; Herb.: GH; Other lists: NPL, SOL; Notes: Not revised for Flora of Madeira.

=49. - Galium mollugo Linn. var. angustifolia [Galium productum Lowe (Rubiaceae)]; Mad.; Herb.: GH (BM000829004, BM000829002), SCV; Other lists: NPL, SOL; Notes: The original label states: " in Madera locus saxocis prope rivulos". The General Herbarium sheet also includes two fragments of G. parisiense L. (BM000829003). Determined by N.J. Turland, VI.1993.

=50.- -..--.-- vesiculosum Mscr. [Galium verrucosum Huds. (Rubiaceae)]; Nat.; Herb.: GH (BM000829036); Other lists: NPL, SOL; Notes: Determined by N.J. Turland, VI.1993.

=51.- Plantago lanceolata Linn. [Plantago lagopus L. (Plantaginaceae)]; Nat.; Herb.: GH; Other lists: NPL, SOL; Notes: Determined by J.R. Press, VIII.1997, for Flora of Madeira.

=52.- ------- media Linn. Tanxage Lus. [Plantago major L. (Plantaginaceae)]; Intr.; Herb.: GH; Other lists: NPL, SOL.

=53.- -.---- albicans Linn. [Plantago lagopus L. and P. lanceolata L. (Plantaginaceae)]; Intr.; Herb.: GH; Other lists: NPL, SOL; Notes: According to J.R. Press the sheet includes one specimen of Plantago lanceolata L. and another one of P. lagopus L, determinated on VIII.1991.

=54.- Eleagnus angustifolia Linn. Oleveira Lus. [Olea maderensis (Lowe) Rivas Mart. \& del Arco (Oleaceae)]; Mad.; Herb.: GH; Other lists: NPL, SOL; Notes: Not revised for Flora of Madeira.

=55.- Ilex Perado Mss. Perado Lus. fig. pict. [Ilex perado Aiton subsp. perado (Aquifoliaceae)]; Mad.; Herb.: GH (BM000536466), SCV; Other lists: NPL, SOL; Notes: Not revised for Flora of Madeira. 
=56.- + --.---- Azevinho Mss. Azevinho Lus. fig. pict. [Ilex canariensis Poir. (Aquifoliaceae)]; Mac.; Herb.: SCV; Other lists: NPL, SOL; Notes: Not revised for Flora of Madeira.

=57.- Sagina procumbens Linn. [Sagina procumbens L. (Caryophyllaceae)]; Nat.; Herb.: GH (BM000072032); Other lists: NPL, SOL; Notes: Determined by M.J. West, I.1992.

Pentandria

=58.- Heliotropium europaeum Linn. [Heliotropium europaeum L. (Boraginaceae)]; Nat.; Herb.: GH; Other lists: NPL, SOL; Notes: Not revised for Flora of Madeira.

=59.- Myosotis scorpioides palustris Linn. [Myosotis secunda Al. Murray (Boraginaceae)]; Nat.; Herb.: GH; Other lists: NPL, SOL; Notes: Determined by M.J. West, II.1991.

=60.- - Nat.; Other lists: NPL, SOL.

=61. - Cynoglossum cheirifolium Linn. Masarogueira Lus. [Cynoglossum creticum Mill. (Boraginaceae)]; Nat.; Other lists: NPL, SOL; Notes: Not revised for Flora of Madeira.

=62.- -.---.-- officinale Linn. [Cynoglossum creticum Mill. (Boraginaceae)]; Nat.; Herb.: GH; Other lists: NPL, SOL; Notes: Not revised for Flora of Madeira. =63.- Echium vulgare Linn. [Echium plantagineum L. (Boraginaceae)]; Intr.; Herb.: GH; Other lists: NPL, SOL.

=64.- Anagallis arvensis Linn. [Anagallis arvensis L. (Primulaceae)]; Nat.; Herb.: GH; Other lists: NPL, SOL; Notes: Not revised for Flora of Madeira.

=65.- Convolvulus flexuosus Mss. [Convolvulus althaeoides L. (Convolvulaceae)]; Nat.; Herb.: GH; Other lists: NPL, SOL; Notes: Examined by Lowe. Not revised for Flora of Madeira.

=66.- *-.-.-.-- Batatas Linn. Batata Lus. [Ipomoea batatas (L.) Lam. (Convolvulaceae)]; Cult.; Other lists: NPL, SOL.

=67.- ------- arvensis Linn. corriola Lus. [Convolvulus arvensis L. (Convolvulaceae)]; Nat.; Herb.: GH; Other lists: NPL, SOL; Notes: Not revised for Flora of Madeira.

=68.- ------ altheoides Linn. [Convolvulus althaeoides L. (Convolvulaceae)]; Nat.; Herb.: GH; Other lists: NPL, SOL; Notes: Not revised for Flora of Madeira. =69.- Campanula erinus Linn. [Campanula erinus L. (Campanulaceae)]; Nat.; Other lists: SOL; Notes: Not revised for Flora of Madeira.

=70.- Lonicera caprifolium Linn. [Lonicera caprifolium L. (Caprifoliaceae)]; Cult.; Other lists: NPL, SOL.

=71.- Mirabilis Jalapa Linn. Boninas Lus. [Mirabilis jalapa L. (Nyctaginaceae)]; Intr.; Herb.: GH; Other lists: NPL, SOL; Notes: Determined by J.R. Press, VII.1987, for Flora of Madeira.

=72.- Datura Stramonium Linn. Bufareira Lus. [Datura stramonium L. (Solanaceae)];

Intr.; Other lists: NPL, SOL. 
=73.- Hyosciamus albus Linn. Maimiendro Lus. [Hyoscyamus albus L. (Solanaceae)]; Nat.; Other lists: NPL, SOL.

=74.- Nicotiana Tabacum Linn. Erva santa Lus. [Nicotiana tabacum L. (Solanaceae)];

Intr.; Herb.: GH (BM000940645); Other lists: NPL, SOL; Notes: Not revised for Flora of Madeira.

=75. ${ }^{*}$ Solanum tuberosum Linn. [Solanum tuberosum L. (Solanaceae)]; Cult.; Other lists: SOL.

=76.- -.------ nigrum Linn. [Solanum americanum Mill. (Solanaceae)]; Nat.; Herb.: GH; Other lists: NPL, SOL; Notes: Determinated by Sandra Knapp, 2008.

77.- -------- pseudocapsicum Linn. Pimenteira Lus. [Solanum pseudocapsicum L. (Solanaceae)]; Intr.; Other lists: NPL, SOL.

=78.- -.--- Lycopersicum Linn. Tomatos Lus. [Lycopersicon esculentum Mill. (Solanaceae)]; Intr.; Herb.: GH; Other lists: NPL, SOL.

=79.- Capsicum frutescens $\alpha$ Linn. [Capsicum annuum L. (Solanaceae)]; Cult.; Herb.: GH; Other lists: NPL, SOL; Notes: Determined by J.M. Eylmons, 16.II. 2005.

=80.- Heberdenia excelsa Mscr. Aderno Lus. fig. pict. [Heberdenia bahamensis (Gaertn.) Sprague (Primulaceae)]; Mac.; Herb.: GH, SCV; Other lists: NPL, SOL; Notes: Not revised for Flora of Madeira.

=81. Lycium barbarum Linn. Espinheiro Lus. [Lycium europaeum L. (Solanaceae)];

Nat.; Herb.: GH (BM001019044); Other lists: NPL, SOL; Notes: Not revised for Flora of Madeira.

=82. $-{ }^{*}$ Mangifera indica Linn. Mangoira Lus. [Mangifera indica L. (Anacardiaceae)];

Cult.; Other lists: NPL, SOL.

$=83 .-{ }^{*}$ Ribes rubrum Linn. [Ribes rubrum L. (Grossulariaceae)]; Cult.; Other lists: SOL.

=84.- *.-.-.--- grossularia Linn. [Ribes grossularia L. (Grossulariaceae)]; Cult.;

Other lists: NPL, SOL.

=85.- Hedera helix Linn. [Hedera maderensis K. Koch ex A.Rutherf. (Araliaceae)];

Mad.; Other lists: NPL, SOL.

$=86 .{ }^{*}$ Vitis vinifera Linn. [Vitis vinifera L. (Vitaceae)]; Intr.; Other lists: NPL, SOL. =87.- Achyranthes aspera Sicula Linn. [Achyranthes sicula (L.) All. (Amaranthaceae)];

Nat.; Herb.: GH, SCV; Other lists: NPL, SOL; Notes: Not revised for Flora of Madeira.

$=88 .-{ }^{*}$ Celosia cristata Linn. [Celosia argentea L. (Amaranthaceae)]; Cult.; Other lists: SOL.

$=89 .-{ }^{*}$ Gardenia florida Linn. [Gardenia jasminoides J. Ellis (Rubiaceae)]; Cult.

=90.- Chenopodium murale Linn. [Chenopodium murale L. (Chenopodiaceae)];

Nat.; Herb.: GH; Other lists: NPL, SOL; Notes: Determined by J.R.

Press, 3.VIII.1987, for Flora of Madeira.

=91.- -.------album Linn. [Chenopodium album L. (Chenopodiaceae)]; Nat.; Herb.:

SCV; Other lists: NPL, SOL. 
=92.- -.-----ambrosioides Linn. [Chenopodium ambrosioides L. (Chenopodiaceae)];

Nat.; Herb.: GH; Other lists: NPL, SOL; Notes: Confirmed by J.R. Press, 4.VIII.1987, for Flora of Madeira.

=93.- Caucalis arvensis Mss. [Torilis arvensis (Huds.) Link subsp. neglecta (Spreng.) Thell. (Apiaceae)]; Nat.; Herb.: GH; Other lists: NPL, SOL; Notes: Determinated by M. J. Cannon, 1989.

=94.- Daucus visnaga Linn. Bisnaga Lus. [Ammi visnaga (L.) Lam. (Apiaceae)]; Nat.; Herb.: GH, SCV; Other lists: NPL, SOL; Notes: Determinated by M.J. Cannon, 1989.

=95.- Ammi majus Linn. Margaca Lus. [Ammi majus L. (Apiaceae)]; Nat.; Other lists: NPL, SOL.

=96.- Sium nodiflorum? Linn. Rabassa Lus. [Apium nodiflorum (L.) Lag. (Apiaceae)]; Intr.; Herb.: GH; Other lists: NPL, SOL; Notes: Not revised for Flora of Madeira.

=97.- Anethum Foeniculum Linn. Funcho Lus. [Foeniculum vulgare Mill. (Apiaceae)];

Nat.; Other lists: NPL, SOL.

=98.- Apium petroselinum Linn. [Petroselinum crispum (Mill.) Fuss (Apiaceae)]; Intr.; Herb.: GH; Other lists: NPL, SOL; Notes: Not revised for Flora of Madeira.

=99.- Rhus coriaria Linn. Sumagre Lus. [Rhus coriaria L. (Anacardiaceae)]; Intr.; Herb.: GH; Other lists: NPL, SOL; Notes: Not revised for Flora of Madeira. =100.- Sambucus ebulus Linn. [Sambucus ebulus L. (Caprifoliaceae)]; Intr.; Other lists: SOL.

=101.- Alsine media Linn. [Stellaria media (L.) Vill. (Caryophyllaceae)]; Nat.; Herb.: $\mathrm{GH}$; Other lists: NPL, SOL.

=102.- Linum perenne Linn. [Linum bienne Mill. (Linaceae)]; Nat.; Herb.: GH (BM000056186), SCV; Other lists: NPL, SOL; Notes: Determinated by M.J. Short, I.1988, for Flora of Madeira.

[Page] 5

Plants of Madeira

HEXANDRIA

=103. ${ }^{*}$ Bromelia Ananas Linn. [Ananas comosus (L.) Merr. (Bromeliaceae)]; Cult.; Other lists: SOL.

=104. - Amaryllis belladonna Linn. [Amaryllis belladonna L. (Amaryllidaceae)]; Intr.; Com.; Other lists: SOL.

=105.- Allium oleraceum Linn. Sabolinho bracco Lus. [Allium dentiferum Webb \&Berthel. (Alliaceae)]; Nat.; Herb.: GH; Other lists: NPL, SOL; Notes: Determined by A. Seregrin, 4.VIII.2003. According to Brullo et al. (2009) the species is present at Madeira.

=106.- *-_-_-_- cepa Linn. [Allium cepa L. (Alliaceae)]; Cult.; Other lists: SOL. =107.- Lilium candidum Linn. [Lilium candidum L. (Liliaceae)]; Cult.; Other lists: SOL.

=108. - Dracaena Draco Linn. [Dracaena draco (L.) L. (Asparagaceae)]; Mac.; Other lists: SOL. 
=109.- Aloe perfoliata Linn. [Aloe vera (L.) Burm.f. (Aloaceae)]; Intr.; Other lists: NPL, SOL.

=110.- *Agave Americana Linn. [Agave americana L. (Agavaceae)]; Intr.; Other lists: NPL, SOL.

=111.- Juncus Effusus Linn. [Juncus effusus L. (Juncaceae)]; Nat.; Herb.: GH, SCV;

Other lists: NPL, SOL; Notes: Not revised for Flora of Madeira.

=112.- -..-.-.-- tenax Mscr. [Juncus inflexus L. (Juncaceae)]; Nat.; Herb.: GH (BM001042040); Other lists: NPL, SOL; Notes: Determined by Sven Snogerup, 1973.

=113. - Meadia repens Mscr. Erva branca. Erva terra. Lus. fig. pict. [Sibthorpia peregrina L. (Scrophulariaceae)]; Mad.; Herb.: GH, SCV; Other lists: NPL, SOL; Notes: Not revised for Flora of Madeira.

=114. Rumex aquaticus Linn. azeda Lus. [Rumex conglomeratus Murray (Polygonaceae)]; Intr.; Herb.: GH (BM00056218); Other lists: NPL, SOL; Notes: Determined without indication of author.

=115.--------- scutatus Linn. Labassa Lus. [Rumex maderensis Lowe (Polygonaceae)]; Mac.; Herb.: SCV; Other lists: NPL, SOL.

=116.- Mermulano Heb. Mss. [Sideroxylon mirmulans R.Br. (Sapotaceae)]; Mad.; Herb.: GH (BM0004398, BM000030980, BM000030981), SCV; Other lists: NPL, SOL; Notes: One of the sheets have the original label: "Mirmulano, Conf.: Heberden”. Revised by T. Leyens \& W. Lobin, VI.1994.

\section{OCTANDRIA}

=117. - Tropaeolum minus Linn. [Tropaeolum majus L. (Tropaeolaceae)]; Cult.; Herb.: GH; Other lists: NPL, SOL; Notes: Not revised for Flora of Madeira. =118. - Vaccinium elevatum Mss. Uveira Lus. [Vaccinium padifolium Sm. (Ericaceae)]; Mad.; Herb.: GH (BM000829320); SCV; Other lists: NPL; Notes: Listed as "Vaccinium exaltatum Mscr." in NPL and SOL. A specimen of this species was located inside the Driying Book (Madeira III bundle), see Fig. 7. =119. - Erica cinerea? Linn. [Erica maderensis (Benth.) Bornm. (Ericaceae)]; Mad.;

Other lists: NPL, SOL.

=120.- Polygonum aviculare Linn. Sempre noiva Lus. [Polygonum aviculare L. (Polygonaceae)]; Nat.; Herb.: SCV; Other lists: NPL, SOL.

=121.- -.----- Hydropiper Linn. Polgueira Lus. [Persicaria hydropiper (L.) Spach (Polygonaceae)]; Nat.; Herb.: GH, SCV; Other lists: NPL, SOL.

=122.- -.-.-- Barbatum Linn. [Persicaria hydropiper (L.) Spach (Polygonaceae)];

Nat.; Herb.: GH, SCV; Other lists: NPL, SOL; Notes: Determinated by J.R. Press as Polygonum hydropiper, 1.I.1990.

\section{ENNEANDRIA}

=123. - Laurus nobilis Linn. [Laurus novocanariensis Rivas Mart. et al. (Lauraceae)]; Mac.; Herb.: GH (BM000829064), SCV; Other lists: NPL, SOL. =124.- -------- indica Linn. [Persea indica (L.) Spreng. (Lauraceae)]; Mac.; Herb.: GH, SCV; Other lists: NPL, SOL; Notes: Not revised for Flora of Madeira. 
=125.- -.----- nitida Mscr. fig. pict. [Apollonias barbujana (Cav.) Bornm. subsp. barbujana (Lauraceae)]; Mac.; Herb.: GH, SCV; Other lists: NPL, SOL; Notes: Determined by M.J. Short, II.94.

=126.- ------- foetens Mscr. fig. pict. Til Lus. [Ocotea foetens (Aiton) Baill. (Lauraceae)]; Mac.; Herb.: GH, SCV; Other lists: NPL, SOL; Notes: Not revised for Flora of Madeira.

=127.- *-_-_-.-- cinamomum Linn. [Cinnamomum verum J.Presl (Lauraceae)]; Cult.; Other lists: SOL.

[Page] 6

Plants of Madeira

DECANDRIA

=128. - Cassia bicapsularis Linn. [Senna bicapsularis (L.) Roxb. (Fabaceae)]; Cult.; Other lists: NPL, SOL.

=129.- Ruta graveolens Linn. Arruda Lus. [Ruta chalepensis L. (Rutaceae)]; Intr.; Herb.: GH (BM000083037); Other lists: NPL, SOL; Notes: Not revised for Flora of Madeira.

=130.- Clethra arborea Mscr. Folhada Lus. fig. pict. [Clethra arborea Aiton (Clethraceae)]; Mad.; Herb.: GH (BM000829091, BM000829092, BM000829093), SCV; Other lists: NPL, SOL; Notes: Aprovavit J.R. Press, 1991.

=131.- Dianthus prolifer Linn. [Petrorhagia nanteuilli (Burnat) P.W. Ball \& Heywood (Caryophyllaceae)]; Nat.; Herb.: GH, SCV; Other lists: NPL, SOL.

=132.- *-.-.-.-- cariophyllus Linn. [Dianthus caryophyllus L. (Caryophyllaceae)]; Cult.; Other lists: NPL, SOL.

=133.- Cucubalus Behen Linn. Estralho Lus. [Silene vulgaris (Moench) Garcke subsp. vulgaris (Caryophyllaceae)]; Nat.; Herb.: SCV; Other lists: NPL, SOL.

=134.- Silene gallica Linn. [Silene gallica L. (Caryophyllaceae)]; Nat.; Herb.: GH (BM000072059), SCV; Other lists: NPL, SOL; Notes: Not revised for Flora of Madeira.

=135.- -------- inaperta Linn. [Silene inaperta L. (Caryophyllaceae)]; Nat.; Herb.: GH; Other lists: NPL, SOL; Notes: Not revised for Flora of Madeira.

=136. - Stellaria graminea Linn. [Stellaria alsine Grimm (Caryophyllaceae)]; Intr.; Herb.: GH, SCV; Other lists: NPL, SOL; Notes: Not revised for Flora of Madeira.

=137. - Cotyledon umbilicus $q$ Linn. [Umbilicus rupestris (Salisb.) Dandy (Crassulaceae)]; Nat.; Herb.: GH; Other lists: NPL, SOL; Notes: Not revised for Flora of Madeira.

=138.- Oxalis corniculata Linn. Pedepassaro, Bolsa de pastor Lus. [Oxalis corniculata L. (Oxalidaceae)]; Intr.; Herb.: GH, SCV; Other lists: NPL, SOL; Notes: Determined by A. Lousteig, 1974, as var. villosa (M. Bieb.) Hohen.

=139. - Cerastium viscosum Linn. [Cerastium glomeratum Thuill. (Caryophyllaceae)]; Nat.; Other lists: NPL, SOL.

$=140 .-{ }^{*}$ Phytolacca decandra Linn. [Phytolacca americana L. (Phytolaccaceae)]; Intr.; Other lists: SOL. 


\section{DODECANDRIA}

=141.- Portulaca oleracea Linn. Baldruegas Lus. [Portulaca oleracea L. subsp. oleracea (Portulacaceae)]; Nat.; Herb.: GH; Other lists: NPL, SOL; Notes: Revised by M.J. Short, VI.1990, for Flora of Madeira.

=142.- Lythrum Hyssopifolia Linn. [Lythrum hyssopifolia L. (Lythraceae)]; Nat.; Herb.: GH, SCV; Other lists: NPL, SOL.

=143.- Agrimonia eupatoria Linn. [Agrimonia eupatoria L. (Rosaceae)]; Nat.; Herb.: GH; Other lists: NPL, SOL; Notes: Not revised for Flora of Madeira.

=144.- Reseda luteola Linn. [Reseda luteola L. (Resedaceae);]; Intr.; Herb.: GH; Other lists: NPL, SOL; Notes: species historically cultivated for dyeing. Not revised for Flora of Madeira.

=145.- Euphorbia peplus Linn. [Euphorbia peplus L. (Euphorbiaceae)]; Nat.; Herb.: SCV; Other lists: NPL, SOL.

=146.- -------- verrucosa Linn. Truivisco Lus. [Euphorbia platyphyllos L. (Euphorbiaceae)]; Nat.; Herb.: GH, SCV; Other lists: NPL, SOL; Notes: Revised by J.M. Short, 9.VII.1987, for Flora of Madeira.

=147. - Sempervivum arboreum Linn. [Aichryson divaricatum (Aiton) Praeger (Crassulaceae)]; Mad.; Other lists: NPL, SOL; Notes: Revised by Lowe.

ICOSANDRIA

=148.- Cactus Ficus indica Linn. [Opuntia ficus-barbarica A. Berger (Cactaceae)]; Intr.; Other lists: SOL.

=149.- ${ }^{*}$ Psidium pyriferum Linn. [Psidium guajava L. (Myrtaceae)]; Cult.; Other lists: NPL, SOL.

$=150 .{ }^{*}$ Eugenia Jambos Linn. [Syzygium jambos (L.) Alston (Myrtaceae)]; Cult.; Herb.: GH; Other lists: NPL, SOL; Notes: Determined by N.J. Turland, IV.1994.

=151.- Myrtus communis Lusitanica Linn. Myrto Lus. [Myrtus communis L. subsp. communis (Myrtaceae)]; Nat.; Other lists: NPL, SOL.

=152.- *-_-_-_-- tarentina Linn. [Myrtus communis L. subsp. tarentina (L.) Nyman (Myrtaceae)]; Cult.; Other lists: NPL, SOL.

=153.- *--.----- pulposa Mss. [Eugenia uniflora L. (Myrtaceae)]; Cult.; Herb.: GH; Other lists: NPL, SOL; Notes: Listed as "Eugenia pulposa Mscr." in NPL and SOL. Not revised for Flora of Madeira.

=154.- Punica granatum Linn. [Punica granatum L. (Punicaceae)]; Cult.; Other lists: NPL, SOL.

=155.- *Amygdalus persica Linn. [Prunus persica (L.) Batsch (Rosaceae)]; Cult.; Other lists: SOL.

=156. ${ }^{*}$ Prunus armenaica Linn. [Prunus armeniaca L. (Rosaceae)]; Cult.; Other lists: SOL.

=157.- *-_--.--- cerasus Linn. [Prunus cerasus L. (Rosaceae)]; Intr.; Other lists: SOL. 
=158.- *-_-.--- domestica Linn. [Prunus domestica L. (Rosaceae)]; Cult.; Other lists: NPL, SOL.

=159. ${ }^{*}$ Mespilus germanica Linn. [Mespilus germanica L. (Rosaceae)]; Cult.; Other lists: SOL.

=160. ${ }^{*}$ Pyrus communis Linn. [Pyrus communis L. (Rosaceae)]; Cult.; Other lists: SOL.

=161.- *-------- Malus Linn. [Malus pumila Mill. (Rosaceae)]; Cult.; Other lists: SOL.

=162.- *-.-.-.-- Cydonia Linn. [Cydonia oblonga Mill. (Rosaceae)]; Cult.; Other lists: SOL.

=163. ${ }^{*}$ Rosa gallica Linn. flore albo. [Rosa gallica L. (Rosaceae)]; Cult.; Other lists: NPL, SOL.

=164. - Rubus pedatus Mss. Silva Lus. [Rubus serrae Solano (Rosaceae)]; Mad.; Herb.: GH (BM00099774); Other lists: NPL, SOL; Notes: Taxonomy follows Matzke Hajek et al. (2016). Determined by N.J. Turland, III.1993 as $R$. grandifolius Lowe.

=165.- -------- fruticosus Linn. [Rubus ulmifolius Schott (Rosaceae)]; Nat.; Herb.: SCV; Other lists: NPL, SOL.

$=166 .{ }^{*}$ Fragaria vesca Linn. [Fragaria vesca L. subsp. vesca (Rosaceae)]; Nat.; Other lists: NPL, SOL.

=167.- Potentilla reptans Linn. [Potentilla reptans L. (Rosaceae)]; Nat.; Herb.: SCV; Other lists: NPL, SOL.

POLYANDRIA

=168. - Chelidonium majus Linn. Seredonia/Celedonia Lus. [Chelidonium majus L. (Papaveraceae)]; Nat.; Herb.: GH; Other lists: SOL; Notes: Not revised for Flora of Madeira.

=169.- Delphinium consolida Linn. [Consolida ajacis (L.) Schur (Ranunculaceae)]; Intr.; Other lists: SOL.

=170.- Nigella damascena Linn. [Nigella damascena L. (Ranunculaceae)]; Intr.; Other lists: NPL, SOL.

$=171 .{ }^{*}$ Liriodendrum tulipifera Linn. [Liriodendron tulipifera L. (Magnoliaceae)]; Cult.; Other lists: SOL.

=172.- Ranunculus repens Linn. Solda da terra. Lus. [Ranunculus repens L. (Ranunculaceae)]; Nat.; Herb.: GH, SCV; Other lists: NPL, SOL; Notes: Confirmed by J.R. Press, X.1986.

Didynamia

=173. - Lavandula pinnata Mss. Criceta de gallo fig. pict. [Lavandula pinnata L.f. (Lamiaceae)]; Mac.; Herb.: GH (BM000829060); Other lists: NPL, SOL; Notes: Confirmed by J.R. Press, I.1994.

=174.- Sideritis canariensis Linn. [Sideritis candicans Aiton (Lamiaceae)]; Mad.; Other lists: NPL, SOL; Notes: Listed as "Sideritis candicans" in SOL. 
=175.- Mentha pulegium Linn. Poejo Lus. [Mentha pulegium L. (Lamiaceae)]; Nat.; Herb.: SCV; Other lists: NPL, SOL.

=176.- ------ rotundifolia Linn. [Mentha suaveolens Ehrh. (Lamiaceae)]; Nat.;

Herb.: GH; Other lists: NPL, SOL; Notes: Determined by J.R. Press, X.1993, for Flora of Madeira.

=177.- Stachys arvensis Linn. [Stachys arvensis (L.) L. (Lamiaceae)]; Nat.; Herb.:

GH; Other lists: NPL, SOL; Notes: Confirmed by J.R. Press, 7.I.1992, for Flora of Madeira.

=178.- Ballota nigra Linn. [Ballota nigra L. (Lamiaceae)]; Nat.; Other lists: NPL, SOL.

=179.- Clinopodium vulgare Linn. [Clinopodium vulgare L. (Lamiaceae)]; Nat.; Other lists: NPL, SOL.

[Page] 8

Plants of Madeira

=180.- Origanum creticum Linn. Ouregas Lus. [Origanum vulgare L. subsp. virens (Hoffmanns. \& Link) Ietsw. (Lamiaceae)]; Nat.; SCV; Other lists: NPL, SOL.

=181.- *Thymus vulgaris Linn. [Thymus vulgaris L. (Lamiaceae)]; Cult.; Other lists: SOL.

=182.- Melissa calamintha Linn. [Calamintha nepeta (L.) Savi subsp. sylvatica (Bromf.) R. Morales (Lamiaceae)]; Nat.; Herb.: GH (BM000778843), SCV; Other lists: NPL, SOL; Notes: Revised by J.R. Press, I. 1992, for Flora of Madeira and determinated as C. sylvatica subsp. ascendens.

=183. . Dracocephalum canariense Linn. [Cedronella canariensis (L.) Webb \& Berthel. (Lamiaceae)]; Mac.; Herb.: GH; Other lists: NPL, SOL; Notes: Not revised for Flora of Madeira.

=184. ${ }^{*}$ Ocymum Basilicum Linn. [Ocimum basilicum L. (Lamiaceae)]; Cult.; Other lists: SOL.

=185.- Prunella vulgaris Linn. [Prunella vulgaris L. (Lamiaceae)]; Nat.; Other lists: NPL, SOL.

=186.- Antirrhinum cordatum Mss fig. pict. Orelhas de gato Lus. [Kickxia elatine (L.) Dumort. (Scrophulariaceae)]; Nat.; Herb.: GH, SCV; Other lists: NPL, SOL; Notes: Not revised for Flora of Madeira.

=187.- Scrophularia scorodonia Linn. [Scrophularia scorodonia L. (Scrophulariaceae)]; Nat.; GH, SCV; Other lists: NPL, SOL; Notes: Revised by Vilhem Dalgaard, 1979.

=188.- Digitalis purpurea Linn. Tegeira Lus. [Digitalis purpurea L. (Scrophulariaceae)]; Nat.; Other lists: NPL, SOL.

=189.- ${ }^{*}$ Bignonia radicans Linn. [Campsis radicans (L.) Seem. (Bignoniaceae)]; Cult.; Other lists: SOL.

=190.- Acanthus spinosus Linn. [Acanthus mollis L. (Acanthaceae)]; Intr.; Herb.: GH; Other lists: SOL; Notes: Revised for Flora of Madeira, M.J. Short, VI.1990. 
Tetradynamia

=191.- ${ }^{*}$ Lepidium sativum Linn. [Lepidium virginicum L. (Brassicaceae)]; Intr.; Herb.: GH; Other lists: NPL, SOL; Notes: In the original label sativum is crossed and changed into virginicum. Revised by M.J. West, XI. 1990, for Flora of Madeira.

=192.- Cochlearia coronopus Linn. [Lepidium coronopus (L.) Al-Shehbaz (Brassicaceae)]; Intr.; Other lists: NPL, SOL.

=193.- Sisymbrium Nasturtium $\nabla$. Linn. Agriao Lus. [Rorippa nasturtium-aquaticum (L.) Hayek (Brassicaceae)]; Nat.; Herb.: GH; Other lists: NPL, SOL; Notes: Revised by M.J. West, XII. 1990 for Flora of Madeira.

=194. - Erysimum officinale Linn. [Sisymbrium officinale (L.) Scop. (Brassicaceae)]; Nat.; Herb.: GH; Other lists: NPL, SOL; Notes: Revised by M.J. Short, IX.1989, for Flora of Madeira.

=195.- ${ }^{*}$ Brassica oleracea Linn. [Brassica oleracea L. (Brassicaceae)]; Cult.; Other lists: NPL, SOL.

=196.- *.-.-.--- rapa Linn. [Brassica rapa L. (Brassicaceae)]; Cult.; Other lists: SOL.

=197.- ${ }^{*}$ Sinapis alba Linn. mustarda Lus. [Sinapis arvensis L. (Brassicaceae)]; Cult.;

Herb.: GH (BM00056580); Other lists: NPL, SOL; Notes: Revised by Guy Baillargeon, 1983.

=198. - Isatis tinctoria Linn. [Isatis tinctoria L. (Brassicaceae)]; Intr.; Herb.: GH;

Other lists: NPL, SOL; Notes: Not revised for Flora of Madeira.

Monadelphia

=199.- Geranium robertianum Linn. Agulheta Lus. [Geranium robertianum L. (Geraniaceae)]; Nat.; Herb.: GH; Other lists: NPL, SOL; Notes: Not revised for Flora of Madeira.

=200.- -------- cicutarium Linn. [Erodium moschatum (L.) Aiton (Geraniaceae)]; Nat.; Herb.: GH; Other lists: NPL, SOL; Notes: Determined by M.J. West, VIII.1990.

=201.- Sida Rhombifolia Linn. Bertonha Lus. [Sida rhombifolia L. (Malvaceae)]; Intr.; Herb.: SCV; Other lists: NPL, SOL.

=202. --.---- [Sida spinosa L. (Malvaceae)] Intr.; Herb.: SCV; Other lists: NPL, SOL.

=203.- Malva rotundifolia Linn. Malva Lus. [Malva sylvestris L. (Malvaceae)]; Nat.; Herb.: GH, SCV; Other lists: NPL, SOL; Notes: Determined by J.R. Press, VI.1980.

=204.- ------- mauritanica Linn. [Malva sylvestris L. (Malvaceae)]; Nat.; Herb.: GH; Other lists: NPL, SOL; Notes: Labeled as Malva mauritiana by B\&S. Determined by J.R. Press, VII.1986.

=205.- ${ }^{*}$ Hibiscus rosa sinensis Linn. [Hibiscus rosa-sinensis L. (Malvaceae)]; Cult.; Other lists: SOL. 
Diadelphia

=206. - Fumaria officinalis Linn. Molharinha Lus. [Fumaria muralis Sond. ex W.D.J. Koch subsp. muralis (Fumariaceae)]; Nat.; Other lists: NPL, SOL;

Notes: Determined by J. Press, 8.X.1989, for Flora of Madeira.

=207.- Spartium scoparium Linn. [Cytisus scoparius (L.) Link subsp. scoparius (Fabaceae)]; Intr.; GH (BM000056343); Other lists: NPL, SOL; Notes: Not revised for Flora of Madeira.

=208.- Genista canariensis Linn. Piorno Lus. [Genista tenera (Murray) Kuntze (Fabaceae)]; Mad.; Herb.: GH; Other lists: NPL, SOL; Notes: Genista canariensis L. (accepted name Teline canariensis (L.) Webb \& Berthel.), to which Banks referred this specimen, does not occur in Madeira but is morphologically similar.

=209. ${ }^{*}$ Lupinus albus Linn. [Lupinus albus L. subsp. albus (Fabaceae)]; Cult.; Other lists: SOL.

=210.- ${ }^{*}$ Phaseolus vulgaris $\alpha$ Linn. [Phaseolus vulgaris L. (Fabaceae)]; Cult.; Other lists: SOL.

=211.- *-.----- $\beta$ coccineus Linn. [Phaseolus coccineus L. (Fabaceae)]; Cult.; Other lists: SOL.

=212. ${ }^{*}$ Pisum sativum Linn. [Pisum sativum L. (Fabaceae)]; Cult.; Other lists: SOL.

=213. - Vicia gracilis Mss. [Vicia articulata Hornm. (Fabaceae)]; Nat.; Herb.: GH

(BM000829338); Other lists: NPL, SOL; Notes: According to Lowe (1868) it refers to Ervum pubescens DC. (=Vicia pubescens (DC.) Link).

=214. - Ervum hirsutum Linn. Ervilhata Lus. [Vicia hirsuta (L.) Gray (Fabaceae)];

Nat.; Herb.: GH; Other lists: NPL, SOL; Notes: Not revised for Flora of Madeira.

=215.- Cicer arietinum Linn. [Cicer arietinum L. (Fabaceae)]; Cult.; Herb.: GH; Other lists: NPL, SOL.

=216- Ornithopus perpusillus Linn. [Ornithopus pinnatus (Mill.) Druce. (Fabaceae)];

Nat.; Herb.: GH, SCV; Other lists: NPL, SOL; Notes: Revised by N.J. Turland, X.1993.

=217.- Scorpiurus vermiculata Linn. [Scorpiurus vermiculatus L. (Fabaceae)]; Nat.;

Other lists: SOL.

=218. - Psoralea bituminosa Linn. Fudigocos Lus. [Bituminaria bituminosa (L.) C.H. Stirton]; Nat.; Herb.: GH (BM000828994); Other lists: NPL, SOL; Notes: Not revised for Flora of Madeira.

=219.- ------- americana Linn. [Cullen americanum (L.) Rydb. (Fabaceae)]; Intr.; Herb.: GH (BM000838996); Other lists: NPL, SOL; Notes: Not revised for Flora of Madeira.

=220.- Trifolium glomeratum Linn. [Trifolium glomeratum L. (Fabaceae)]; Nat.; Herb.: GH; Other lists: NPL, SOL; Notes: Not revised for Flora of Madeira. =221.- Trifolium agrarium Linn. [Trifolium dubium Sibth. (Fabaceae)]; Nat.; Herb.: GH (BM000056746), SCV; Other lists: NPL, SOL; Notes: Determined by R.J. Press, II.1987, for Flora of Madeira. 
=222.- -.-.--- repens Linn. [Trifolium repens L. (Fabaceae)]; Nat.; Herb.: GH, SCV; Other lists: NPL, SOL; Notes: Confirmed by J.R. Press, III.1987, for Flora of Madeira.

=223.- -------- Melilotus Italica Linn. [Melilotus elegans Salzm. ex Ser. (Fabaceae)]; Intr.; Other lists: NPL, SOL.

=224.- ------- angustifolium Linn. Pesegaja Lus. [Trifolium angustifolium L. (Fabaceae)]; Nat.; Herb.: GH (BM000829021), SCV; Other lists: NPL, SOL; Notes: Confirmed by R.J. Press, II.1987, for Flora of Madeira.

=225.- Lotus glaucus Mscr. fig. pict. [Lotus glaucus Aiton (Fabaceae)]; Mad.; Herb.: GH (BM00050789), SCV; Other lists: NPL, SOL; Notes: Determined by J.H. Kirkbride Jr., 2006.

=226.- Medicago polymorpha muricata Linn. [Medicago polymorpha L. (Fabaceae)];

Nat.; Herb.: GH, SCV; Other lists: NPL, SOL; Notes: Not revised for Flora of Madeira.

=227.- -------- lupulina Linn. [Medicago lupulina L. (Fabaceae)]; Nat.; Other lists: NPL, SOL.

POLYADELPHia

$=228 .-{ }^{*}$ Citrus medica Linn. [Citrus medica L. (Rutaceae)]; Cult.; Other lists: SOL. =229.- -.-.--- Aurantium Linn. [Citrus $x$ aurantium L. (Rutaceae)]; Cult.; Other lists: SOL.

=230.- Hypericum canariense Linn. Sepiao Lus. [Hypericum canariense L. (Hypericaceae)]; Mac.; Herb.: GH; Other lists: NPL, SOL; Notes: Determined by N.K.B. Robson, 1980 .

=231.- -------- perforatum Linn. peseguiero Lus. [Hypericum perforatum L. (Hypericaceae)]; Nat.; GH, SCV; Other lists: NPL, SOL; Notes: Determined by N.K.B. Robson as var. veronense (Scrank) H. Lindb., 1998.

=232.- ---.-- humifusum Linn. [Hypericum humifusum L. (Hypericaceae)]; Nat.; Herb.: GH, SCV; Other lists: NPL, SOL; Notes: Determined by N.K.B. Robson, 2007.

=233.- ------- glandulosum Mscr. [Hypericum glandulosum Aiton (Hypericaceae)]; Mac.; Herb.: GH (BM000617916); Other lists: NPL, SOL; Notes: Determined by N.K.B. Robson, 1996.

=234.- -------- evectum Mscr. [Hypericum grandifolium Choisy (Hypericaceae)]; Mac.; Herb.: GH (BM000829215, BM0001024250); Other lists: NPL, SOL; Notes: Determinated by N.K.B. Robson, 1981.

[Page] 10

Plants of Madera

Syngenesia

=235.- Picris Echioides Linn. Rapasaya Lus. [Helminthotheca echioides (L.) Holub (Asteraceae)]; Nat.; Herb.: GH, SCV; Other lists: NPL, SOL; Notes: Determined by J.R. Press, V.1990, for Flora of Madeira.

=236. - Sonchus oleraceus Linn. Peseguiro Lus. [Sonchus asper (L.) Hill (Asteraceae)]; Nat.; Herb.: SCV; Other lists: NPL, SOL. 
=237.-Lactuca sativa Linn. [Lactuca sativa L. (Asteraceae)]; Cult.; Herb.: GH; Other lists: NPL, SOL; Notes: Not revised for Flora of Madeira.

=238. - Leontodon nudicaule Mss. Letubra Lus. [Leontodon taraxacoides (Vill.) Mérat (Asteraceae)]; Nat.; Herb.: GH, SCV; Other lists: NPL, SOL; Notes: Determinated by J.R. Press, IV.1990.

=239.- Crepis tenuifolia Mss. [Tolpis succulenta (Aiton) Lowe (Asteraceae)]; Mac.; Herb.: GH, SCV; Other lists: NPL, SOL; Notes: Revised by C.E. Jarvis. =240.- Lapsana stellata Linn. [Rhagadiolus stellatus (L.) Gaertn. (Asteraceae)]; Intr.;

Herb.: GH; Other lists: NPL, SOL; Notes: Determined by P.D. Sell, 1972. =241.- Cicoreum Intybus Linn. [Cichorium endivia L. subsp. divaricatum (Schousb.) P.D.Sell (Asteraceae)]; Nat.; Herb.: GH (BM000017576); Other lists: NPL, SOL.

=242.- Scolymus hispanicus Linn. [Scolymus maculatus L. (Asteraceae)]; Nat.; Other lists: NPL, SOL.

=243.- Arctium Lappa Linn. [Arctium minus (Hill.) Bernh. (Asteraceae)]; Intr.;

Other lists: SOL.

=244. - Carduus pycnocephalus Linn. [Galactites tomentosa Moench (Asteraceae)];

Nat.; Herb.: SCV; Other lists: NPL, SOL; Notes: Specimen in SCV labeled Carduus pycnocephalus corresponds to Galactites tomentosa. Not revised for Flora of Madeira.

=245. - Carthamus tinctorius Linn. Cardo brabo Lus. [Carthamus tinctorius L. (Asteraceae)]; Intr.; Other lists: NPL, SOL.

=246.- Carthamus lanatus Linn. [Carthamus lanatus L. subsp. lanatus (Asteraceae)];

Nat.; Herb.: GH; Other lists: NPL, SOL; Notes: Aprovavit J.R. Press, 1991. =247.- Gnaphalium crassifolium Mss [crossed] fig. pict. [Helichrysum obconicum

DC. (Asteraceae)]; Mad.; Herb.: GH (BM000829047, BM00829048),

SCV; Other lists: NPL, SOL; Notes: Determined by J.R. Press, IX.1994, for Flora of Madeira.

=248.- ------- luteoalbidum Linn. [Pseudognaphalium luteoalbum (L.) Hilliard \&

B.L. Burtt (Asteraceae)]; Nat.; Herb.: GH (BM000829049), SCV; Other lists: NPL, SOL; Notes: Determined by J.R. Press, V.1991.

=249.- Conyza Saxatilis Linn. Murnaneira Lus. [Phagnalon saxatile (L.) Cass. (Asteraceae)]; Herb.: GH, SCV; Nat.; Other lists: NPL, SOL; Notes: Determined by J.R. Press, I.1990.

=250. - Erigeron canadense Linn. [Conyza canadensis (L.) Cronquist (Asteraceae)];

Intr.; Herb.: GH, SCV; Other lists: NPL, SOL; Notes: Determined by J.R. Press, I.1990.

=251.- Senecio viscosus Linn. [Senecio lividus L. (Asteraceae)]; Nat.; Herb.: GH

(BM000829050, BM000829051), SCV; Other lists: NPL, SOL; Notes:

Determined by J.W. Kadereit, 17.VIII.1982.

=252. ${ }^{*}$ Aster chinensis Linn. [Callistephus chinensis (L.) Nees. (Asteraceae)]; Cult.;

Other lists: SOL.

$=253 .{ }^{*}$ Tagetes erecta Linn. [Tagetes erecta L. (Asteraceae)]; Cult.; Other lists: SOL. =254. - Chrysanthemum segetum Linn. [Glebionis segetum (L.) Fourr. (Asteraceae)];

Intr.; Other lists: SOL. 
=255.- Matricaria parthenium Linn. Artemigo Lus. [Tanacetum parthenium (L.) Sch.Bip. (Asteraceae)]; Intr.; Herb.: GH; Other lists: NPL, SOL; Notes: Determined by J.R. Press, 21.XI.1988, for Flora of Madeira.

=256.- Anthemis cotula Linn. [Anthemis cotula L. (Asteraceae)]; Intr.; Other lists: NPL, SOL.

$=257 .-{ }^{*}$ Helianthus annuus Linn. [Helianthus annuus L. (Asteraceae)]; Cult.; Other lists: SOL.

=258.- Coreopsis Leucanthema Linn. Malpica / Don Andrera Lus. [Bidens pilosa L. var. radiata (Sch.Bip.) Sherff (Asteraceae)]; Intr.; Herb.: GH, SCV; Other lists: NPL, SOL; Notes: Determined by T.G.J. Raynaer, 1983.

=259.- Centaurea calcitrapa Linn. [Centaurea calcitrapa L. (Asteraceae)]; Nat.; Herb.: GH (BM000847062); Other lists: NPL, SOL; Notes: Determined by J.R. Press, III.1991.

=260.- Calendula arvensis Linn. [Calendula arvensis L. (Asteraceae)]; Nat.; Other lists: NPL, SOL.

=261.- Lobelia Longifolia Mscr. [Legousia scabra (Lowe) Gamisans (Campanulaceae)]; Nat.; Herb.: GH, SCV; Other lists: NPL, SOL.

[Page] 11

Plants of Madeira

=262.- Viola odorata Linn. Viola Lus. [Viola odorata L. (Violaceae)]; Nat.; Herb.: GH, SCV; Other lists: NPL, SOL; Notes: Revised for Flora of Madeira by M.J. Short, II.1990.

=263. ${ }^{*}$ Impatiens balsamina Linn. [Impatiens balsamina L. (Balsaminaceae)]; Intr.; Other lists: SOL.

GYNANDRIA

=264.- Passiflora quadrangularis Linn. [Passiflora quadrangularis L. (Passifloraceae)]; Intr.; Other lists: SOL; Notes: cultivated species with edible fruits.

=265. ${ }^{*}$ Arum colocasia Linn. Inhame Lus. [Colocasia esculenta (L.) Schott (Araceae)];

Intr.; GH; Other lists: NPL, SOL; Notes: Determined by E.W. Groves, VI.1972.

=266.- ------- sagittifolium Linn. [Arum italicum Mill. subsp. canariense (Webb \& Berthel.) P.C.Boyce (Araceae)]; Mac.; Herb.: GH; Other lists: NPL, SOL; Notes: Determined by N.J. Turland, XII.1993.

=267.- -.---.-- pictum Mss Inhame de Bresil Lus. fig. pict. [Caladium picturatum K. Koch \& C.D. Bouché (Araceae)]; Cult.; Herb.: GH; Other lists: NPL, SOL.

Monoecia

=268.- Lemna minor Linn. [Lemna minor L. (Lemnaceae)]; Nat.; Other lists: SOL. =269.- Zea Mays Linn. [Zea mays L. (Poaceae)]; Cult.; Other lists: SOL.

=270.- Coix Lacryma Jobi Linn. Conteiras brancas Lus. [Coix lacryma-jobi L. (Poaceae)]; Cult.; Herb.: GH, SCV; Other lists: NPL, SOL; Notes: Confirmed by T.A. Cope, 7.XI.1988. 
=271.- Carex muricata Linn. [Carex divulsa Stokes subsp. divulsa (Cyperaceae)]; Nat.; Herb.: GH; Other lists: NPL, SOL; Notes: Determined by Rene David, 15.IV.1993.

=272.- *Buxus sempervirens Linn. [Buxus sempervirens L. (Buxaceae)]; Cult.; Other lists: SOL.

=273.- Urtica urens Linn. [Urtica membranacea Savigny (Urticaceae)]; Nat.; Herb.: GH (BM000072973); Other lists: NPL, SOL; Notes: Determined by J.R. Press, 24.II.1987, for Flora of Madeira.

$=274$. ${ }^{*}$ Morus nigra Linn. [Morus nigra L. (Moraceae)]; Cult.; Other lists: SOL. =275. - Amaranthtus hybridus Linn. [Amaranthus hybridus L. (Amaranthaceae)]; Intr.; Herb.: SCV; Other lists: NPL, SOL.

=276.- -.--.-- blitum Linn. Bredos Lus. [Amaranthus blitum L. (Amaranthaceae)];

Nat.; Herb.: GH (BM000083055), SCV; Other lists: NPL, SOL; Notes:

Not revised for Flora of Madeira.

=277.- Poterium sanguisorba Linn. [Sanguisorba verrucosa (Link ex G.Don) Ces.

(Rosaceae)]; Nat.; Herb.: GH (BM000035955); Other lists: NPL, SOL;

Notes: Not revised for Flora of Madeira. This is a sterile specimen.

=278.- Juglans regia Linn. [Juglans regia L. (Juglandaceae)]; Cult.; Other lists: SOL. =279.- Fagus castanea Linn. [Castanea sativa Mill. (Fagaceae)]; Intr.; Other lists: SOL.

=280.- Pinus pinea Linn. Pinheira. [Pinus pinaster Aiton. (Pinaceae)]; Intr..; Herb.: GH; Other lists: NPL, SOL.

$=281 .{ }^{*}$ Cupressus sempervirens Linn. [Cupressus sempervirens L. (Cupressaceae)]; Cult.; Other lists: SOL.

=282.- ${ }^{*}$ Cucurbita lagenaria Linn. [Lagenaria siceraria (Molina) Standl. (Cucurbitaceae)]; Cult.; Other lists: SOL.

=283.- *--.----- pepo Linn. [Cucurbita pepo L. (Cucurbitaceae)]; Cult.; Other lists: SOL.

=284.- *.-.-.--- citrullus Linn. [Citrullus lanatus (Thunb.) Matsum. \& Nakai $(\mathrm{Cu}-$ curbitaceae)]; Cult.; Other lists: SOL.

$=285-{ }^{*}$ Cucumis Melo Linn. [Cucumis melo L. (Cucurbitaceae)]; Intr.; Other lists: SOL.

=286.- *.-...-.- Sativus Linn. [Cucumis sativus L. (Cucurbitaceae)]; Intr.; Other lists: SOL.

[Page] 12

Plants of Madera

Dioecia

=287.- Salix purpurea Linn. Vime Lus. [Salix x rubens Schrank (Salicaceae)]; Cult.; Herb.: GH, SCV; Other lists: NPL, SOL; Notes: Determined by R.J. Press, I.1991,

=288.- -.---.-- Helix Linn. Seiseiro Lus. [Salix x rubens Schrank (Salicaceae); vime, seixo]; Cult.; Herb.: GH; Other lists: NPL, SOL.

=289.- Smilax latifolia Mss Alegacadela Lus. fig. pict. [Smilax pendulina Lowe (Smilacaceae)]; Mad.; Herb.: GH (BM001009926, BM001009927), SCV; 
Other lists: NPL, SOL; Notes: Determined as Smilax aspera by A.R. Vickery, 6.X.1989.

=290.- Populus alba Linn. Alamo Lus. [Populus alba L. (Salicaceae)]; Intr.; Herb.: GH, SCV; Other lists: NPL, SOL; Notes: Not revised for Flora of Madeira. =291.- Mercurialis ambigua Linn. [Mercurialis annua L. (Euphorbiaceae)]; Nat.; Herb.: GH, SCV; Other lists: NPL, SOL; Notes: Determinated by J.R. Press, VII.1986, for Flora of Madeira.

=292.- ${ }^{*}$ Carica Papya Linn. [Carica papaya L. (Caricaceae)]; Cult.; Other lists: SOL. =293.- Juniperus oxycedrus Linn. [Juniperus cedrus Webb \& Berthel. subsp. maderensis Rivas Mart. et al. (Cupressaceae)]; Mad.; Herb.: GH; Other lists: NPL, SOL; Notes: Determined by R.J. Press, II.1991.

=294.- Taxus baccata Linn. [Taxus baccata L. (Taxaceae)]; Nat.; Herb.: GH; Other lists: NPL, SOL; Notes: Aprovavit R.J. Press, 1991.

$=295 .-{ }^{*}$ Ruscus androgynus Linn. Alegacam Lus. [Semele androgyna (L.) Kunth (Liliaceae)]; Mac.; Herb.: GH; Other lists: NPL, SOL; Notes: Determined by A.R. Vickery, 5.VI.1989.

POLYGamia

=296.- Musa sapientium Linn. [Musa acuminata Colla (Musaceae)]; Cult.; Other lists: SOL.

=297.- Andropogon hirtum Linn. [Hyparrhenia hirta (L.) Stapf (Poaceae)]; Nat.; Herb.: GH, SCV; Other lists: NPL, SOL; Notes: Determined by O.S.

=298.- Holcus halepensis Linn. Scalracha Lus. [Sorghum halepense (L.) Pers. (Poaceae)]; Intr.; Herb.: GH, SCV; Other lists: NPL, SOL; Notes: Not revised for Flora of Madeira.

=299.- -..--.-- mollis Linn. [Holcus lanatus L. (Poaceae)]; Intr.; Herb.: GH (BM000643301), SCV; Other lists: NPL, SOL; Notes: In original label: "Madera locis saxosis prope Funchal 14 Sept.1768". Determined by T.A. Cope, 13.IV.1989.

=300.- Cenchrus setosus Mscr. [Cenchrus ciliaris L. (Poaceae)]; Intr.; Herb.: GH, SCV; Other lists: NPL, SOL; Notes: Determined by T.A Cope, 18.V.1988, for Flora of Madeira.

=301.- Parietaria officinalis Linn. Alfavaca Lus. [Parietaria judaica L. (Urticaceae)]; Nat.; Herb.: GH; Other lists: NPL, SOL; Notes: The sheet also appears to have a specimen of $P$. officinalis $\mathrm{L}$. determinated by J.R. Press which probably is not from Madeira.

=302. ${ }^{*}$ Mimosa Farnesiana Linn. [Acacia farnesiana (L.) Willd. (Fabaceae)]; Intr.; Other lists: SOL.

=303. $-{ }^{*}$ Diospyrus Lotus Linn. Fig. Fuit. [Diospyros lotus L. (Ebenaceae)]; Cult.; Herb.: SCV; Other lists: NPL, SOL.

=304. ${ }^{*}$ Ficus carica Linn. [Ficus carica L. (Moraceae)]; Cult.; Other lists: SOL. 
Cryptogamia

=305.- Equisetum arvense Linn. [Equisetum arvense L. (Equisetaceae)]; Nat.; Herb.: GH; Other lists: NPL, SOL.

=306. + +Osmunda Maderensis Mscr. fig. pict. [Pteris incompleta Cav. (Pteridaceae)];

Nat.; Herb.: GH, SCV; Other lists: NPL, SOL; Notes: Revised for Flora of Madeira.

=307.- -.---.-- spicant Linn. [Struthiopteris spicant (L.) F.W.Weiss. (Blechnaceae)];

Nat.; Herb.: GH; Other lists: NPL, SOL; Notes: Revised for Flora of Madeira.

=308.- Pteris aquilina Linn. Feiteira Lus. [Pteridium aquilinum (L.) Kuhn (Hypolepidaceae)]; Nat.; Other lists: NPL, SOL.

=309.- Asplenium adiantum nigrum Linn. [Asplenium adiantum-nigrum L. (Aspleniaceae)]; Nat.; Herb.: SCV; Other lists: NPL, SOL.

=310.- ------- marinum Linn. [Asplenium marinum L. (Aspleniaceae)]; Nat.; Herb.: SCV; Other lists: NPL, SOL.

=311.- -------- monanthes Linn. fig. pict. [Asplenium monanthes L. (Aspleniaceae)]; Nat.; Other lists: NPL, SOL.

[Page] 13

Plants of Madeira

=312.- Polypodium Thelypteris Mscr. Acrostichum Th. Linn. fig. pict. [Christella dentata (Forssk.) Brownsey \& Jermy (Thelypteridaceae)]; Nat.; GH, SCV;

Other lists: NPL, SOL; Notes: Revised for Flora of Madeira.

=313.- ------- lineatum Mscr. [Asplenium billotii F.W. Schultz (Aspleniaceae)]; Nat.;

Herb.: SCV; Other lists: NPL, SOL.

=314.- -.---.--- fragile Linn. [Cystopteris viridula (Desv.) Desv. (Woodsiaceae)]; Nat.;

Herb.: GH; Other lists: NPL, SOL; Notes: Determined by Mary Gibby, 3.II.1989, as C. diaphana (Bory) Blasdell. Revised for Flora of Madeira.

=315.- Adiantum trapeziforme Linn. Cabreira Lus. [Adiantum capillus-veneris L.

(Adiantaceae);]; Nat.; Herb.: SCV; Other lists: NPL, SOL.

=316. - Trichomanes canariense Linn. [Davallia canariensis (L.) Sm. (Davalliaceae)];

Nat.; Herb.: GH, SCV; Other lists: NPL, SOL; Notes: Revised for Flora of Madeira.

=317.- Mnium politrichoides Linn. [Mnium sp. (Mniaceae)]; Nat.; Other lists: NPL, SOL.

=318.- Hypnum rugosum Mscr. [Hypnum sp. (Hypnaceae)]; Nat.; Other lists: NPL, SOL.

=319.- Jungermannia tamariscifolia Linn. [Frullania tamarisci (L.) Dumort. (Jubulaceae)]; Nat.; Other lists: NPL, SOL.

=320.- Lichen articulatus Linn. Barba Lus. [Usnea articulata (L.) Hoffm. (Parmeliaceae)]; Nat.; Other lists: NPL, SOL.

=321.- -.--.--- calicaris Linn. [Ramalina calicaris (L.) Fr. (Ramalinaceae); liquen];

Nat.; Other lists: NPL, SOL.

=322.- ------- digitatus Linn. [Cladonia digitata (L.) Hoffm. (Cladoniaceae)]; Nat.;

Other lists: NPL, SOL. 
=323. - Marchantia polymorpha Linn. Patinha Lus. [Marchantia polymorpha L. (Marchantiaceae)]; Nat.; Other lists: NPL, SOL.

=324.- Lichen geographicus Linn. [Rhizocarpon geographicum (L.) DC. (Rhizocarpaceae)]; Nat.; Other lists: NPL, SOL.

=325.- -...--- calicaris Linn. [Ramalina calicaris (L.) Fr. (Ramalinaceae)]; Nat.; Other lists: NPL, SOL.

=326.- Byssus aurea Linn. [Trentepohlia aurea (L.) Mart. (Trentepohliaceae)]; Nat.; Other lists: NPL, SOL.

=327.- Phoenix dactyfera Linn. [Phoenix dactylifera L. (Arecaceae)]; Intr.; Other lists: SOL; Notes: During Banks and Solander's visit to Madeira the Canary Island palm (P. canariensis $\mathrm{H}$. Wildpret) was not recognized as a distinct species. We cannot rule out that this record refers to this Canary Island endemic.

Appendix

=328.- Faya Lus. [Morella faya (Aiton) Wilbur (Myricaceae)]; Mac.; Herb.: GH, SCV; Other lists: NPL, SOL; Notes: Revised by M.J. Short, VI.1986, for Flora of Madeira.

=329.- Pao branco Lus. [Picconia excelsa (Aiton) DC. (Oleaceae)]; Mac.; Herb.: SCV; Other lists: NPL, SOL; Notes: Not revised for Flora of Madeira.

=330.- Salvia major folio glauco serrate. Sloane Hist. Jam. Pag. 17. T.3 fig.3 [Teucrium betonicifolium Jacq. (Lamiaceae)]; Mad.; Herb.: GH (BM001025250), SCV; Other lists: NPL, SOL; Notes: Species listed as T. betonicum L'Hér. by Press and Short (1994), Jardim and Menezes de Sequeira (2008), and Menezes de Sequeira et al. (2012), see details regarding this name in article by Francisco-Ortega et al. (2020). Not revised for Flora of Madeira.

HERBARIUM SPECIMENS WITHOUT MATCHING ENTRIES IN BANK'S JOURNAL

For the seven species listed below we located herbarium specimens, but they do not correspond to material recorded in Banks's journal list. Only one of these species, the Madeiran endemic Bystropogum punctatus, was recorded in the two other studied documents.

Bystropogon punctatus L'Hér. (Lamiaceae); Mad.; Herb.: GH; Other lists: NPL, SOL.

Galium parisiense L. (Rubiaceae); Nat.; Herb.: GH (BM000829003); Notes: See entry 49 for further details about this species and this specimen.

Isolepis cernua (Vahl) Roem. \& Schult. (Cyperaceae); Nat.; Herb.: GH.

Plantago coronopus L. (Plantaginaceae); Nat.; Herb.: GH. Not revised for Flora of Madeira.

Plantago maritima L. (Plantaginaceae); Nat.; Herb.: GH; Notes: This species is not listed by Press and Short (1994), Jardim and Menezes de Sequeira (2008) or Menezes de Sequeira et al. (2012). Not revised for Flora of Madeira.

Rumex cf. crispus L. (Polygonaceae); Intr.; Herb.: GH.

Tolpis barbata (L.) Gaertn. (Asteraceae); Nat.; Herb.: GH. 


\section{CONCLUDING REMARKS}

Our study of Banks and Solander's collections and records from Madeira stem from three documents found in two different countries: Australia and the United Kingdom. These documents are very similar in their content, but because they had different scopes there are differences among them. Solander's Primitiae Florae Maderensis aimed to provide a tentative flora for the island and includes both plant records from Cook's expedition and a list of Madeiran taxa previously published by the pre-Linnean naturalists Leonard Plukenet and Hans Sloane (Francisco-Ortega et al. 1994; Menezes de Sequeira et al. 2010). The second of these documents, the inventory of specimens placed inside the drying books, only provides an index of the collected herbarium material. It includes details on the number of duplicate collections as well as information on the location of specimens inside the drying books. The third document is part of Banks's journal and it is the only one housed in Australia. This manuscript lists all plants that were recorded in Madeira.

Regarding the BM herbarium specimens, they are found in three different sets, namely: the BM general herbarium, one of the expedition's drying book (Madeira III bundle), and one of the bounded volumes of the BM historical collections that contains specimens collected during the voyage.

When the available information and material found in these three different documents and herbarium holdings is analyzed together we found that Banks's collections from Madeira comprised 334 records for 328 taxa. Twenty of these taxa are Madeiran endemics, 19 are Macaronesian endemics, 156 are non-endemic natives, 67 are introduced, and 72 are cultivated. Among them, 305 taxa are spermatophyte, twelve are ferns, two are mosses, two are liverworts, one is an alga, five are lichens, and one is a fungus. Regarding the herbarium material there are 210 collections for 206 taxa, 16 of these taxa are Madeiran endemics, 18 are Macaronesian endemics, 100 are non-endemic natives, 60 are introduced, and 12 are cultivated. These specimens account for ten fern and 196 spermatophyte taxa; and Banks and Solander primarily focused on collecting natives - both endemics and non-endemics.

\section{ACKNOWLEDGEMENTS}

The GB-TAF of the Synthesys program (www.synthesys.info) is acknowledged for support for a research visit to BM by ASG. This is contribution 377 from the Tropical Biology Program of Florida International University. Our gratitude to Susana Sa Fontinha for her identifications of bryophytes and to Francisco Fernandes for his help with common plant names used in Madeira. Jordan Goodman helped with the bibliography. Miguel Sequeira and an anonymous reviewer critically read the manuscript. The authors thank the organizing committee of FloraMac2018 for sponsoring their attendance to this international meeting. 


\section{AUTHOR'S CONTRIBUTION}

Botanical history review: ASG, JFO, MAC

Location and taxonomic identification of relevant herbarium specimens: ASG, MAC First draft: ASG, JFO

Review and edition of the final draft: all authors.

RECibido: mayo 2020; ACEPTAdo: junio 2020 


\section{BIBLIOGRAPHY}

Aiton, W. 1789a. Hortus Kewensis. Volume 1. London: Georg Nicol 496 pp.

Aiton, W. 1789b. Hortus Kewensis. Volume 3. London: Georg Nicol 547 pp.

Banks, J., Solander, D.C. and Cook, J. 1985. Banks' florilegium. A publication in thirty-four parts of seven hundred and thirty-eight copperplate engravings of plants collected on Captain James Cook's first voyage round the world in H.M.S. Endeavour 1768-1771. Part XIX Madeira plates 391-401. London: Alecto Historical Editions 406 pp.

Beaglehole, J.C. (ed.). 1962a. The Endeavour journal of Joseph Banks 1768-1771. Volume 1. Sidney: Trustees of the Public Library of New South Wales 406 pp.

Beaglehole, J.C. (ed.). 1962b. The Endeavour journal of Joseph Banks 1768-1771. Volume 2. Sidney: Trustees of the Public Library of New South Wales 476 pp.

Britten, J. 1904. R. Brown's list of Madeiran plants. J. Bot. 42: 1-8, 39-46, 175-182, 197-200.

Brullo, S., Guglielmo, A., Pavone, P. and Salmon, C. 2008. Taxonomy study on Allium dentiferum Webb et Berthel. (Alliacaeae) and its relations with allied species from the Mediterranean. Taxon 57(1): 243-253.

Buch, C.L. von. 1825. Physicalische beschreibung der Canarischen Inseln. Berlin: Kön, Akademie der Wissenschaften 407 pp.

Carvalho, P., Figueira, R. and Jones, M.P. 2008. Lista dos líquenes e fungos liquinícolas (fungi). In: Borges, P.A.V., Abreu, C., Aguiar, A.M.F., Carvalho, P., Jardim, R., Melo, I., Oliveira, P., Sérgio, C., Serrano, A.R.M. and Vieira, P. (eds.). A list of the terrestrial fungi, flora and fauna of Madeira and Selvagens arquipelagos. Funchal and Hangra do Heroismo: Direcção Regional do Ambiente do Madeira and Universidade dos Açores pp. 107-122.

Cole, D. 1980. Sigismund Bacstrom's northwest coast drawings and an account of his curious career. BC Studies, 46: 61-86.

De Candolle, A. 1841. Second mémoire sur la famille des Myrsinéacées. Ann. Sci. Nat. 16: 65-97.

De Wit, H.C.D. 1957. Some remarks on Heberdenia A.DC., Pleiomeris A.DC. and Afrardisia Mez (Myrs.). Bull. Jard. Bot. Etat Bruxelles 27: 233-242.

Edwards, P.I. 1979. Sir Joseph Banks and the botany of Captain Cook's three voyages of explorations. Pac. Stud. 2: 20-43.

Francisco-Ortega, J., Santos-Guerra, A. and Jarvis, C. 1994. Pre-Linnaean references for the Macaronesian flora found in Leonard Plukenet's works and collections. Bulletin Natural History Museum, London 24: 1-34.

Francisco-Ortega, J., Santos-Guerra, A., Carine, M., and Jarvis, C. 2008. Plant hunting in Macaronesia by Francis Masson: the plants sent to Linnaeus and Linnaeus filius. Bot. J. Linn. Soc. 157: 393-428.

Francisco-Ortega, J., Santos-Guerra, A., Romeiras, M.M., Carine, M.A., Sánchez-Pinto, L. and Duarte, M.C. 2015. The botany of the three voyages of Captain Cook in Macaronesia: an introduction. Rev. Acad. Canar. Cienc. 27: 357-410.

Francisco-Ortega, J., Gandhi, K.N., Duarte, M.C. Santos-Guerra, A., Carine, M.A. and Romeiras, M.M. 2020. Captain James Cook and Macaronesian botany: typification and nomenclature updates of the six new species described by Johann R. Forster and J. Georg A. Forster. Scientia Insularum 3: 39-54. 
González Lemus, N. 2003. Glosario biográfico de navegantes, naturalistas y viajeros relacionados con el Teide. In: González Lemus, N. (ed.). El Teide. Representación e identidad. Catálogo Exposición 2003. Excmo. Cabildo Insular de Tenerife pp. 191-228.

González Lemus, N. 2012. De los viajeros británicos a Canarias a lo largo de la historia. Anuario de Estudios Atlánticos 58: 51-104.

Goodman, J. 2020. Two hundred and fifty years ago today: the Banksian botanical 'suite' arrives in Madeira on HMS Endeavour. Scientia Insularum 3: 27-33.

Groves, E.W. 1962. Notes on the botanical specimens collected by Banks and Solander on Cook's first voyage, together with an itinerary of landing. Journal of the Society for the Bibliography of Natural History, 4: 57-62.

Heberden, E. 1990. Thomas Heberden 1703-1769. Surgeon, physician and naturalist. Curtis's Bot. Mag. 7: 131-133.

Heberden, T. 1752. Observations made in going up the Pic of Teneriffe. Philos. T. R. Soc. 47: 353-357.

Jardim, R. and Menezes de Sequeira, M. 2008. Lista das plantas vasculares (Pteridophyta and Spermatophyta). In: Borges, P.A.V., Abreu, C., Aguiar, A.M.F., Carvalho, P., Jardim, R., Melo, I., Oliveira, P., Sérgio, C., Serrano, A.R.M. and Vieira, P. (eds.). A list of the terrestrial fungi, flora and fauna of Madeira and Selvagens arquipelagos. Funchal and Angra do Heroismo: Direcçâo Regional do Ambiente do Madeira and Universidade dos Açores pp. 157-207.

L'Héritier, C.L. 1788. Sertum anglicum. Paris: Petri-Francisci Didot 36 pp.

LIAS. 1995-2020. A global information system for lichenized and lichenilocous Aschomycetes. Available from http://www.lias.net/.

Linnaeus, C. 1762. Species plantarum. Volume 1. Second edition. Stockholm: Laurentii Salvii 784 pp.

Linnaeus, C. 1763. Species plantarum. Volume 2. Second edition. Stockholm: Laurentii Salvii 900 pp.

Lobin, W., Leyens, T., Santos, A., Costa Neves, H. and Gomes, I. 2005. The genus Sideroxylon (Sapotaceae) on the Madeira, Canary Islands and Cape Verde archipelagoes. Vieraea 33: 119-144.

Lowe, R.T. 1857-1868. A Manual Flora of Madeira and the adjacent islands of Porto Santo and The Desertas. Vol. 1, part 1, 1857, part 2, 1862, part 3, 1864, part 4, 1868, part 5, 1868; Vol. 2, part 1, 1872. London: John Van Voorst.

Matzke-Hajek, G., Gonçalves Silva, and Paz, R.F. 2016. The four Rubus species of Madeira (Portugal). Bol. Mus. Nat. Hist. Funchal 66: 19-30.

Melo, I. and Cardoso, J. 2008. Lista dos fungos. In: Borges, P.A.V., Abreu, C., Aguiar, A.M.F., Carvalho, P., Jardim, R., Melo, I., Oliveira, P., Sérgio, C., Serrano, A.R.M. and Vieira, P. (eds.). A list of the terrestrial fungi, flora and fauna of Madeira and Selvagens arquipelagos. Funchal and Angra do Heroismo: Direcçâo Regional do Ambiente do Madeira and Universidade dos Açores pp. 73-93.

Menezes de Sequeira, M., Espírito-Santo, D., Aguiar, C., Capelo, J. and Honrado, J. (Coordinators). 2012. Checklist da Flora de Portugal (Continental, Açores e Madeira). Associação Lusitanica de Fitossociologia 74 pp.

Menezes de Sequeira, M., Santos-Guerra A., Jarvis C.E., Oberli A., Carine M.A., Maunder, M. and Francisco-Ortega J. 2010. The Madeiran plants collected by Sir Hans Sloane in 1687, and his descriptions. Taxon 59: 598-612. 
Moore, D.T. 2001. Some aspects of the work of Robert Brown and the Investigator naturalists in Madeira during August 1801. Arch. Nat. Hist. 28: 383-394.

Murray, G. 1904. Botany. In: Anonymous (ed.). The history of the collections contained in the Natural History Departments of the British Museum. Volume 1. London: Trustees of the British Museum pp. 77-193.

Press, J.R. and Short, M.J. (ed.). 1994. Flora of Madeira. London: The Natural History Museum $574 \mathrm{pp}$.

Puig-Samper, M.A. and Pelayo, F. 1997. El viaje del astrónomo y naturalista Louis Feuillée a las Islas Canarias (1724). La Laguna: Centro de la Cultura Popular Canaria, Ayuntamiento de La Laguna 207 pp.

Santos-Guerra, A., Jarvis, C.E., Carine, M.A., Maunder, M. and Francisco-Ortega, J. 2011. Late $17^{\text {th }}$ century herbarium collections from the Canary Islands: The plants collected by James Cuninghame in La Palma. Taxon 60: 1734-1753.

Sérgio, C., Sim-Sim, M., Fontinha, S. and Figueira, R. 2008. Lista dos briófitos (Bryophyta). In: Borges, P.A.V., Abreu, C., Aguiar, A.M.F., Carvalho, P., Jardim, R., Melo, I., Oliveira, P., Sérgio, C., Serrano, A.R.M. and Vieira, P. (eds.). A list of the terrestrial fungi, flora and fauna of Madeira and Selvagens arquipelagos. Funchal and Angra do Heroismo: Direcçâo Regional do Ambiente do Madeira and Universidade dos Açores pp. 143-156.

STÅHL, B. 1996. The relationships of Heberdenia bahamensis and H. penduliflora (Myrsinaceae). Bot. J. Linn. Soc. 122: 315-333.

Sloane, H. 1707. A voyage to the islands Madera, Barbados, Nieves, S. Christophers and Jamaica. Volume 1. London: British Museum 592 pp.

Vieira, R. 1992. Flora da Madeira. O interesse das plantas endémicas Macaronésicas. Lisboa: Servicio de Parques, Reservas e Conservaçáo da Natureza 155 pp.

Vieira, R. 2002. Flora da Madeira. Plantas vasculares naturalizadas no arquipélago da Madeira. Funchal: Museu Municipal do Funchal (História Natural). Suplemento n. ${ }^{\circ}$ 8, 281 pp. 


\section{APPENDIX 1}

Accepted names are printed in bold. Names as they were written in Banks's list (Banks's journal housed in State Library of New South Wales, Australia) are printed in regular font.

Name

ENTRY NUMBER

Acacia farnesiana (L.) Willd... 302

Acanthus mollis L. 190

Acanthus spinosus Linn. 190

Achyranthes aspera Sicula Linn.

Achyranthes sicula (L.) All.

Acrostichum Th. Linn.

Adiantum capillus-veneris $\mathrm{L}$.

Adiantum trapeziforme Linn.

${ }^{*}$ Agave americana $\mathrm{L}$.

${ }^{*}$ Agrimonia eupatoria L.

Agrostis bromoides Linn.

Agrostis linearis Mscr.

Agrostis pallida Mscr..

Agrostis sanguinalis Mscr..

Agrostis sp.

Agrostis sylvatica Linn.

Aichryson divaricatum (Aiton) Praeger

*Allium cepa $\mathrm{L}$. 106

Allium dentiferum Webb \& Berthel. 105

Allium oleraceum Linn. 105

Aloe perfoliata Linn.

Aloe vera (L.) Burm.f.

Alsine media Linn. 101

Alypum angustifolium Mscr.

*Amaranthus blitum L. 276

*Amaranthus hybridus $\mathrm{L}$. 275

${ }^{*}$ Amaryllis belladonna $\mathrm{L}$. 104

*Ammi majus $\mathrm{L}$.

Ammi visnaga (L.) Lam.

Amomun Zingiber Linn. 
Amygdalus persica Linn.

${ }^{*}$ Anagallis arvensis $\mathrm{L}$.

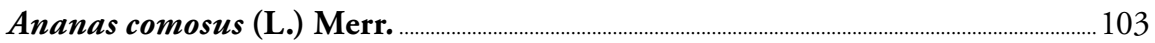

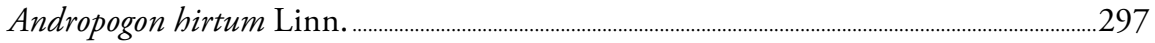

Anethum Foeniculum Linn........................................................................................................................ 97

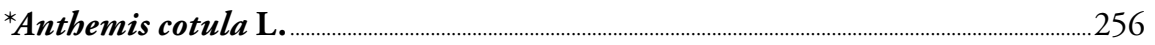

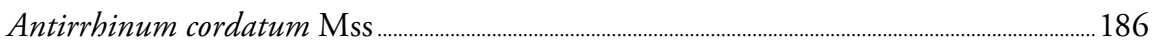

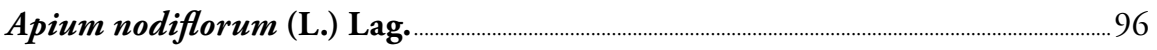

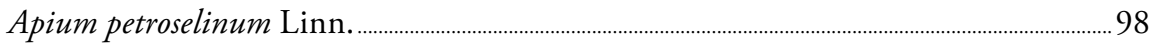

Apollonias barbujana (Cav.) Bornm. subsp. barbujana …………………….................... 125

Arctium Lappa Linn.................................................................................................................................... 243

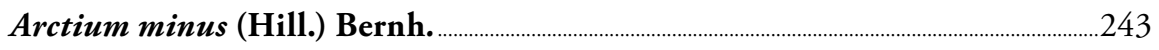

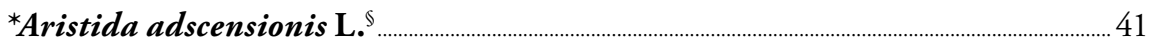

Arrbenatherum elatius (L.) J. Presl \& C. Presl subsp. bulbosum ………………....... 38

Arrhenatherum elatius (L.) J. Presl \& C. Presl

subsp. bulbosum (Willd.) Schübl. \& G. Martens ……………………………………………...... 39

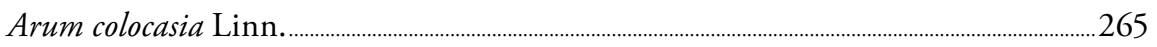

Arum italicum Mill. subsp. canariense (Webb \& Berthel.) P.C. Boyce ………....266

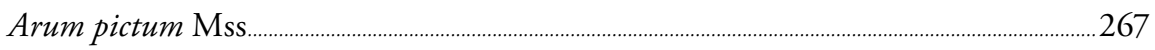

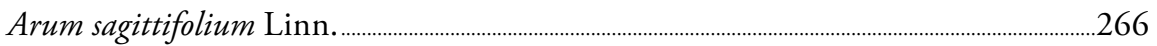

*Arundo donax L. ......................................................................................................................................... 40

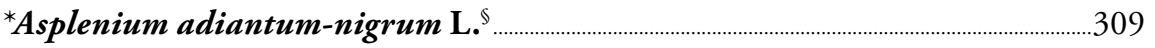

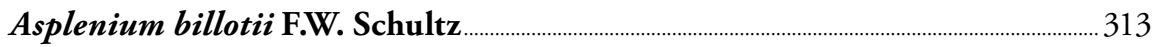

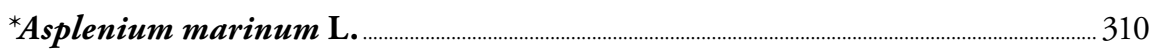

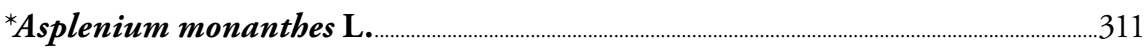

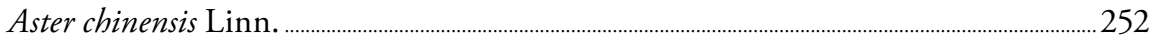

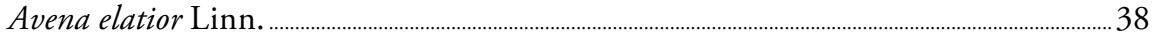

*Avena barbata Pott ex Link …………………….................................................................................... 37

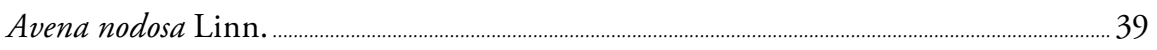

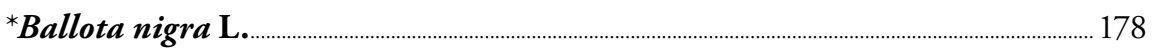

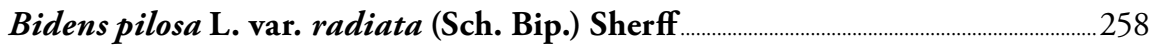

Bignonia radicans Linn.................................................................................................................................... 189

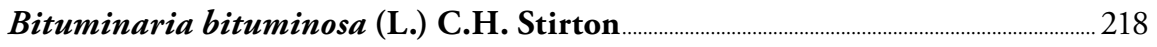

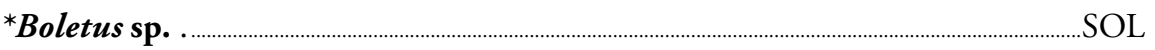

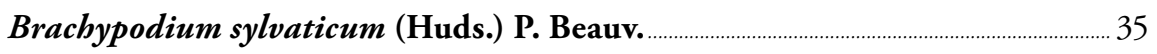

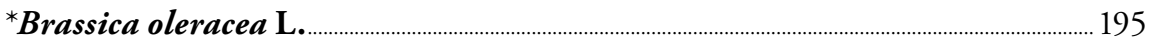

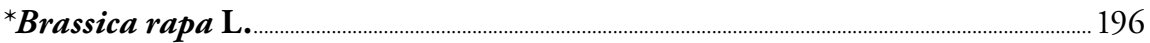




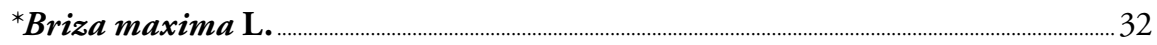

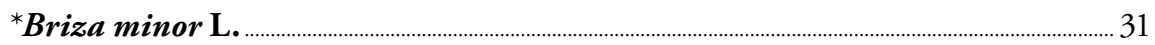

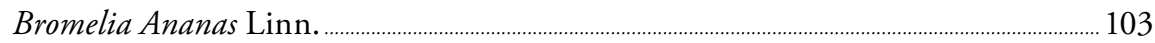

Bromus geniculatus Linn.................................................................................................................................... 36

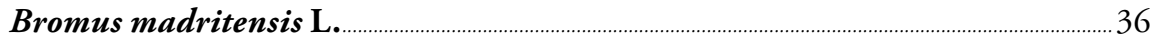

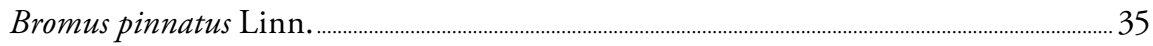

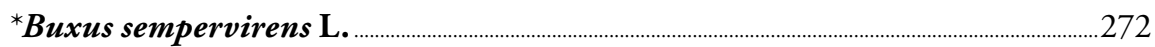

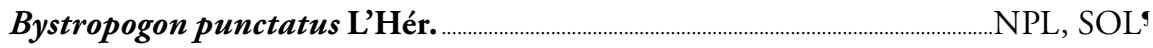

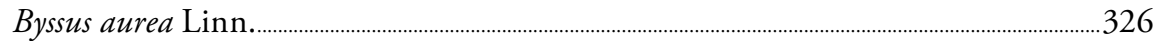

Cactus Ficus indica Linn......................................................................................................................... 148

Caladium picturatum K. Koch \& C.D. Bouché _.......................................................267

Calamintha nepeta (L.) Savi subsp. sylvatica (Bromf.) R. Morales ……................. 182

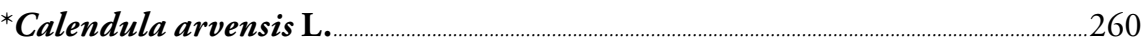

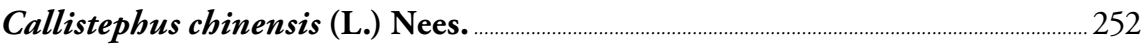

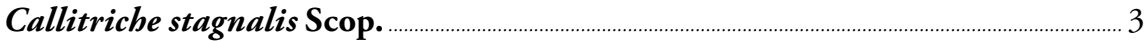

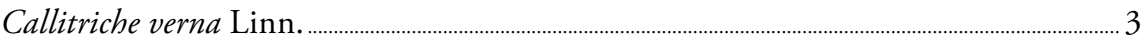

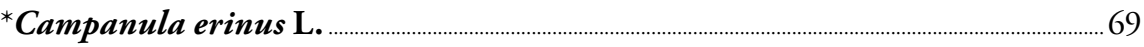

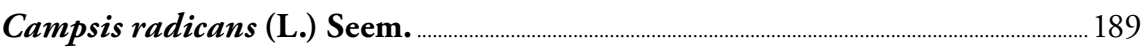

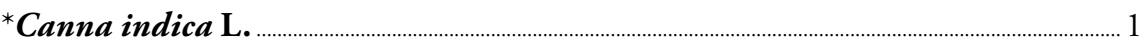

*Capsicum annuum L. .

Carduus pycnocephalus Linn...................................................................................................................24

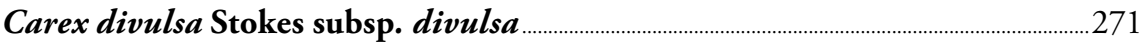

*Carica papaya L. $\$$

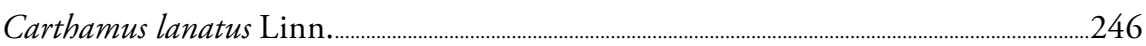

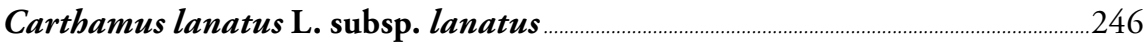

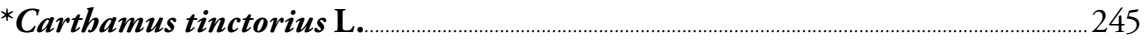

Cassia bicapsularis Linn..................................................................................................................................... 128

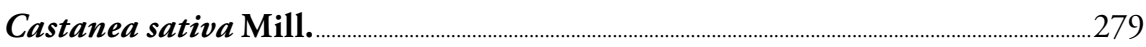

Caucalis arvensis Mss............................................................................................................................... 93

Cedronella canariensis (L.) Webb \& Berthel................................................................. 183

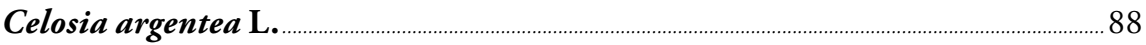

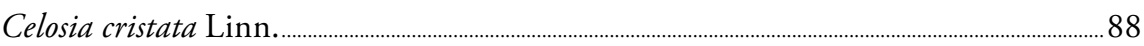

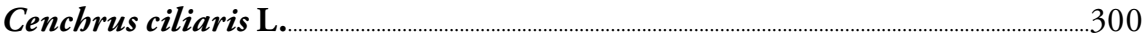

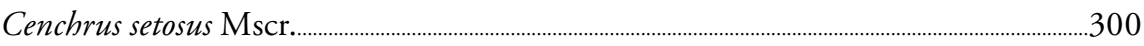

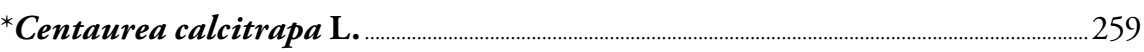

Cerastium glomeratum Thuill...................................................................................................... 139

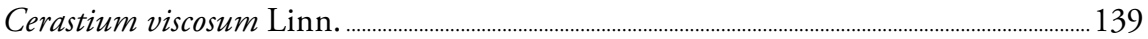




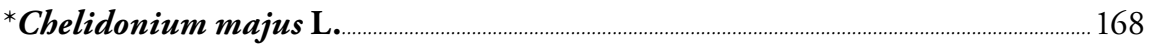

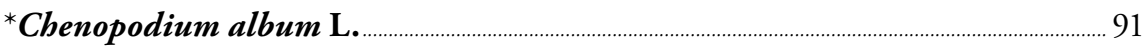

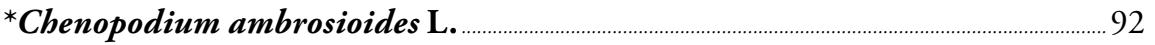

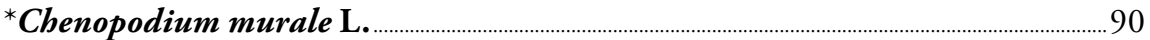

Christella dentata (Forssk.) Brownsey \& Jermy .................................................................. 312

Chrysanthemum segetum Linn. ....................................................................................................................2. 254

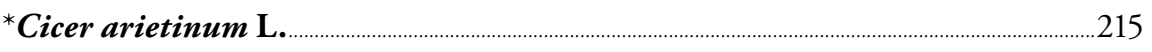

Cichorium endivia L. subsp. divaricatum (Schousb.) P.D. Sell ................................. 241

Cicoreum intybus Linn.............................................................................................................................. 241

Cinnamomum verum J.Presl ..................................................................................................... 127

Citrullus lanatus (Thunb.) Matsum. \& Nakai ............................................................................2 284

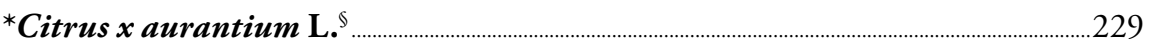

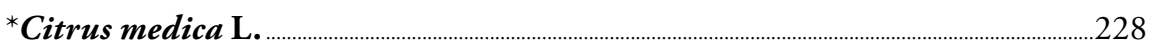

Cladonia digitata (L.) Hoffm.

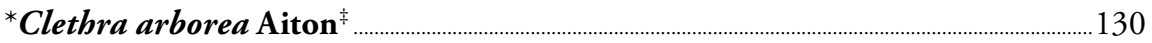

*Clinopodium vulgare L........................................................................................................................ 179

Cochlearia coronopus Linn.................................................................................................................... 192

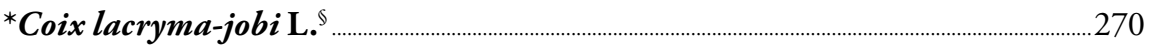

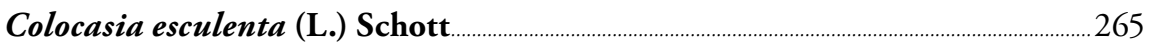

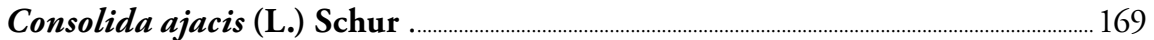

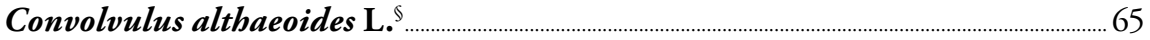

*Convolvulus althaeoides L. .................................................................................................. 68

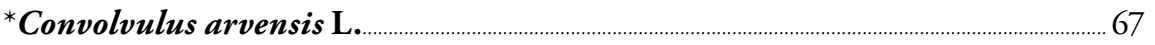

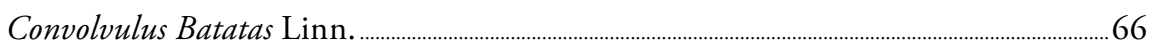

Convolvulus flexuosus Mss. ............................................................................................................................ 65

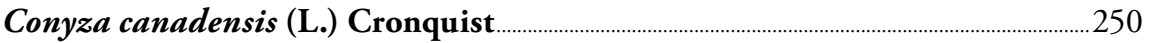

Conyza Saxatilis Linn............................................................................................................................................ 249

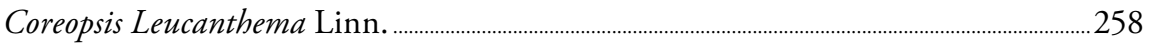

Cotyledon umbilicus Linn.......................................................................................................... 137

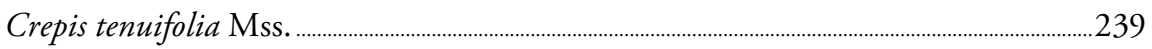

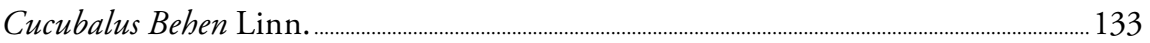

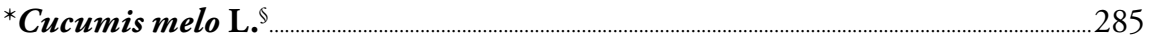

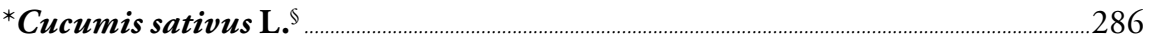

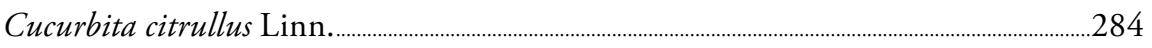

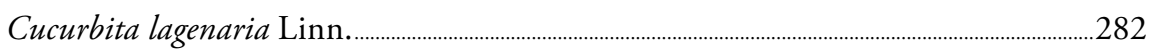

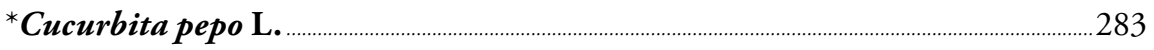

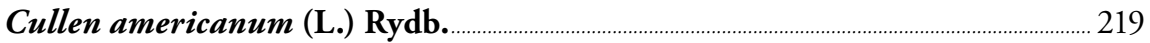




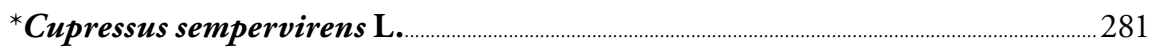

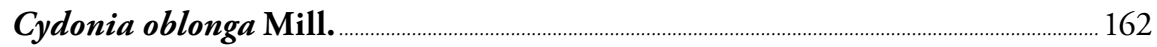

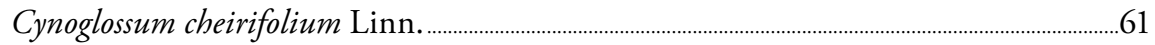

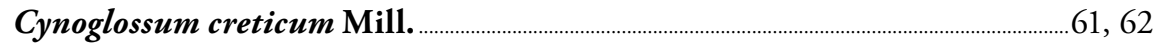

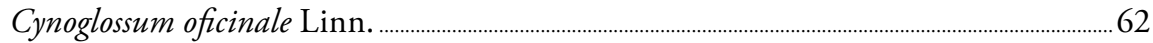

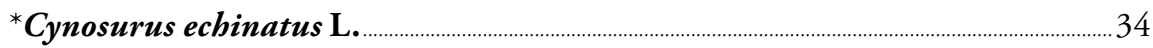

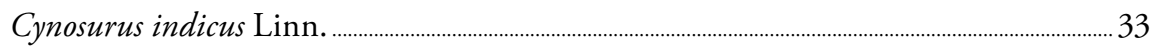

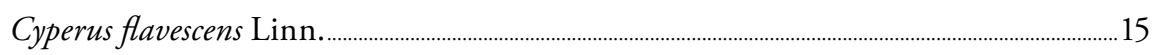

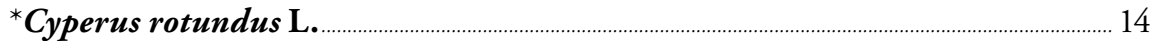

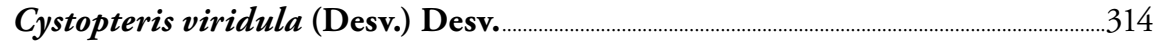

Cytisus scoparius (L.) Link subsp. scoparius ..............................................................................207

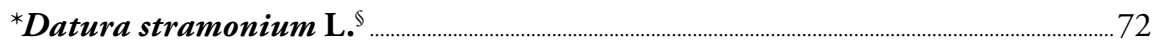

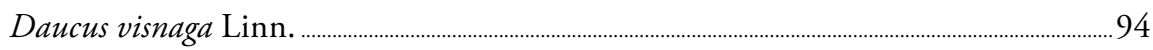

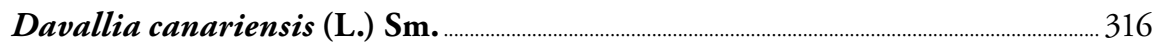

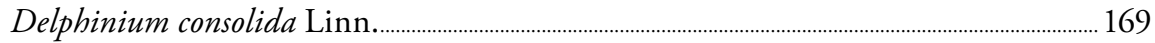

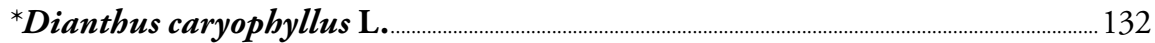

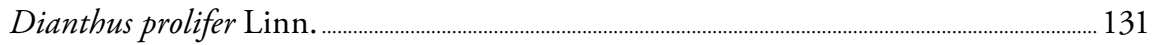

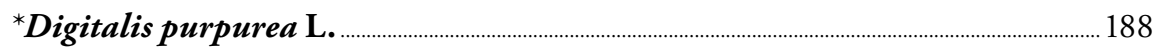

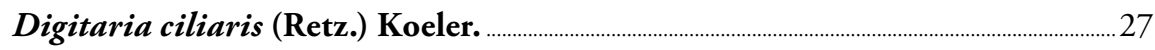

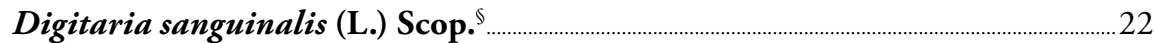

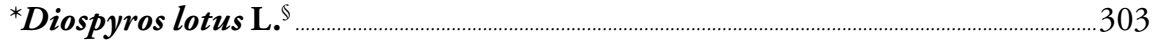

*Dracaena draco (L.) L. ..................................................................................................................... 108

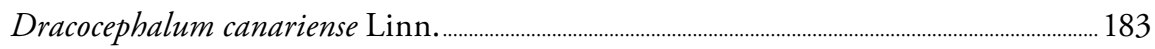

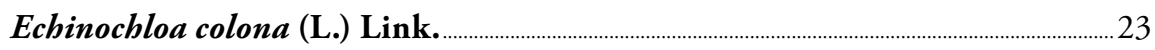

Echium plantagineum L. ........................................................................................................................ 63

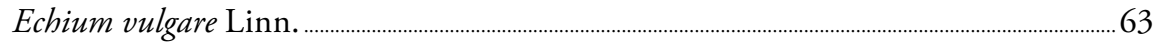

Eleagnus angustifolia Linn............................................................................................................................. 54

Eleusine indica (L.) Gaertn............................................................................................................... 33

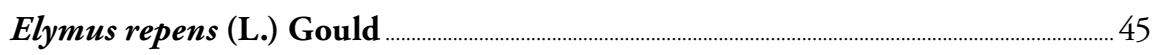

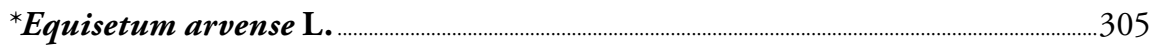

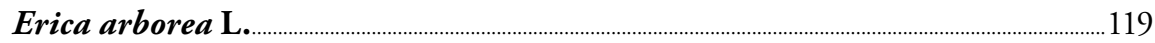

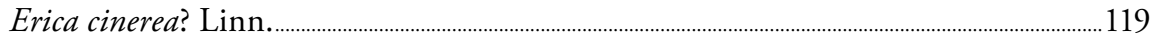

Erigeron canadense Linn.............................................................................................................................. 250

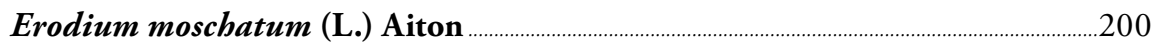

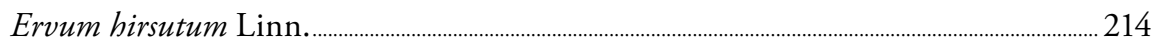

Erysimum officinale Linn........................................................................................................................... 194

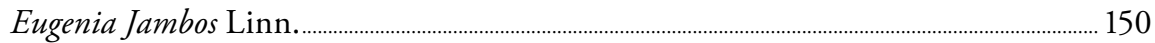




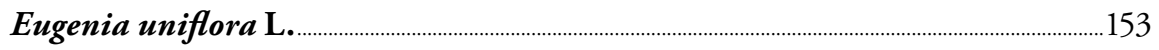

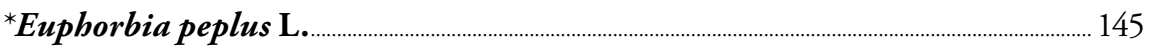

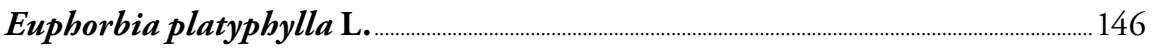

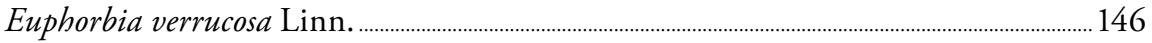

Fagus castanea Linn...........................................................................................................................2.

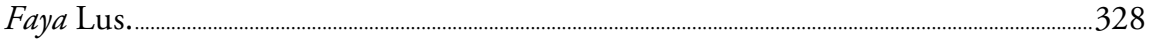

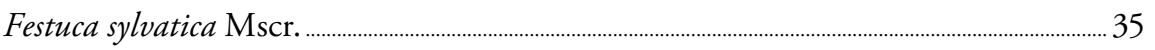

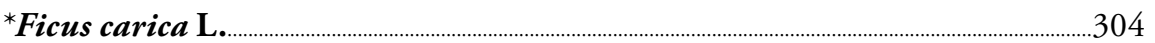

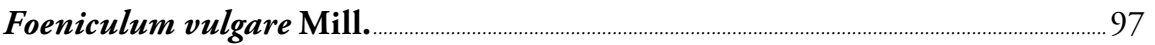

Fragaria vesca L. subsp. vesca ………………………………………………………………………….... 166

Fragaria vesca Linn............................................................................................................................................... 166

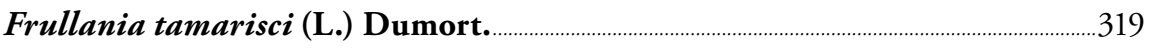

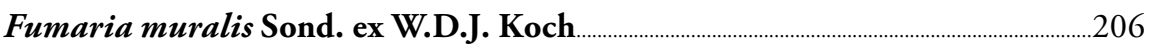

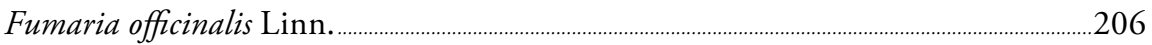

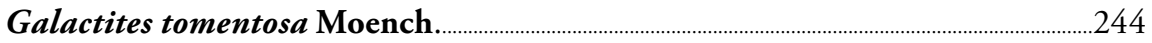

Galium mollugo Linn. var. angustifolia ................................................................................................... 49

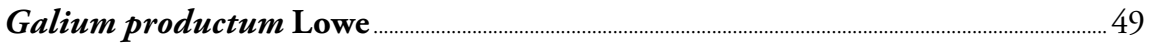

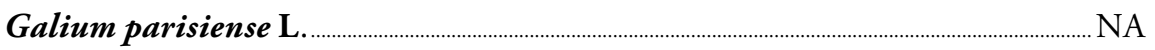

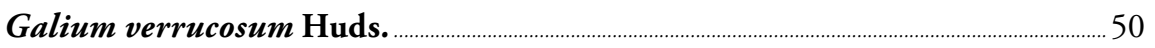

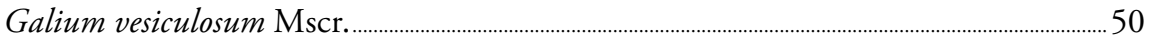

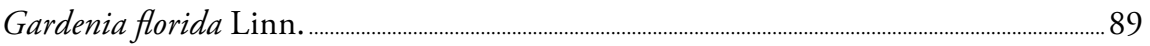

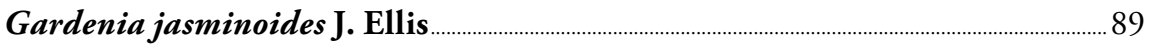

Gastridium ventricosum (Gouan) Schinz \& Thell. ........................................................ 28

Genista canariensis Linn................................................................................................................... 208

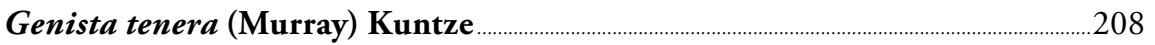

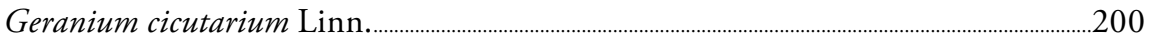

${ }^{*}$ Geranium robertianum L. ..................................................................................................................... 199

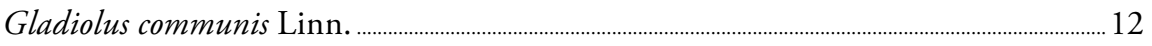

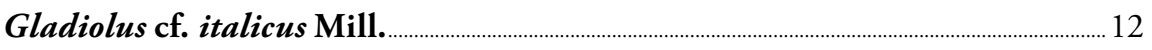

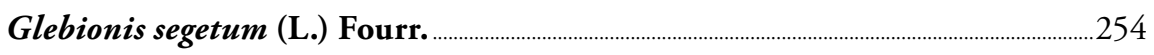

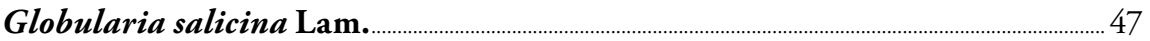

Gnaphalium crassifolium Mss [crossed] ................................................................................................... 247

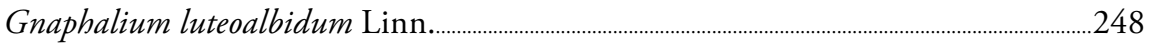

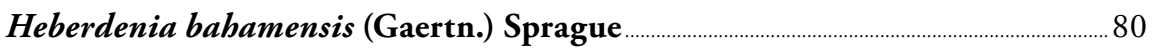

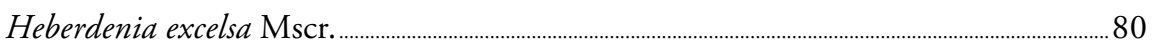

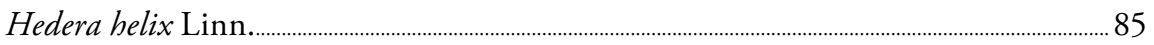

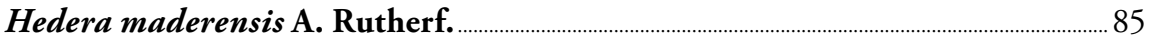




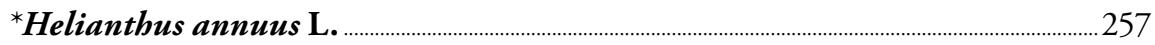

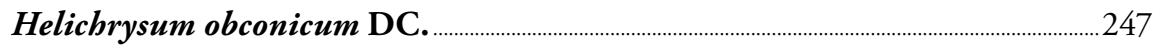

${ }^{*}$ Heliotropium europaeum L. ........................................................................................................ 58

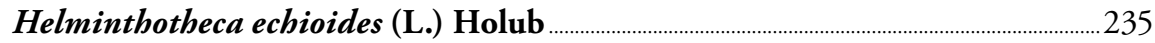

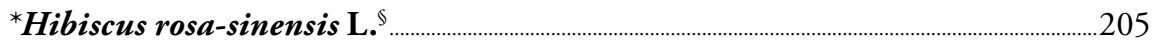

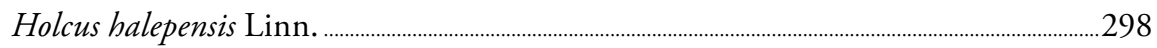

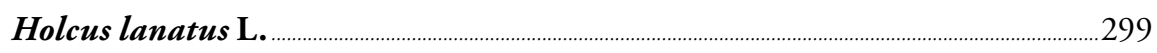

Holcus mollis Linn. ...................................................................................................................................2. 299

${ }^{*}$ Hordeum murinum L. ...................................................................................................................... 44

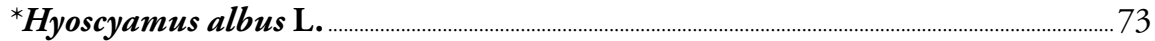

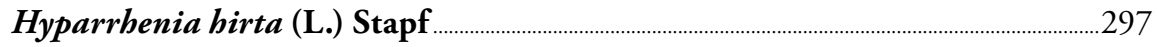

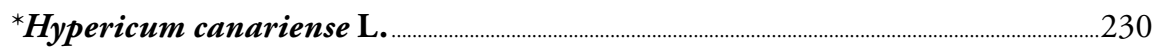

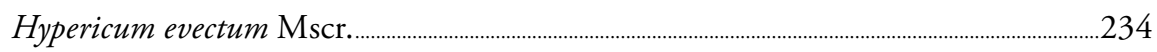

${ }^{*}$ Hypericum glandulosum Aiton …………………………........................................................................ 233

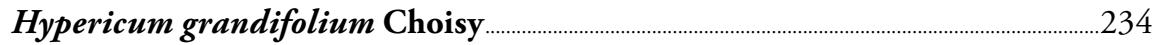

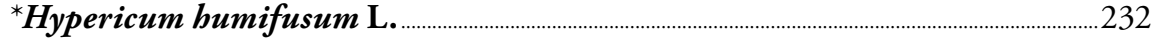

${ }^{*}$ Hypericum perforatum L. ..................................................................................................... 231

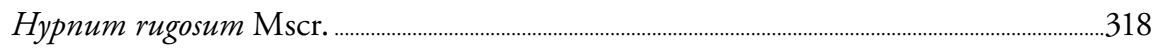

Hypnum sp.

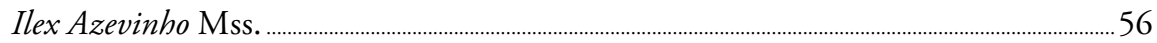

Ilex canariensis Poir.................................................................................................................................. 56

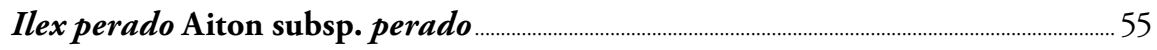

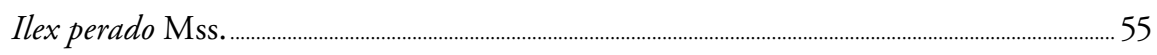

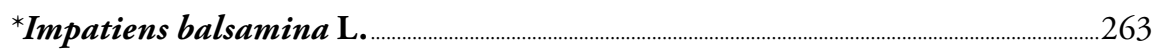

Ipomoea batatas (L.) Lam........................................................................................................................ 66

${ }^{*}$ Iris sp. ${ }^{¥}$.

${ }^{*}$ Isatis tinctoria L....................................................................................................................................... 198

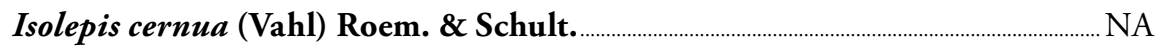

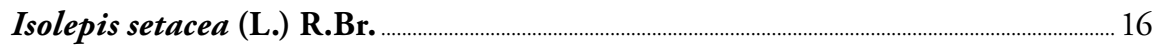

*Jasminum grandiflorum L. .................................................................................................................. 6

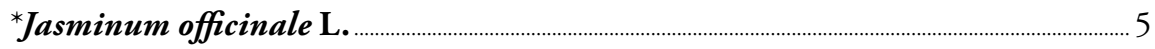

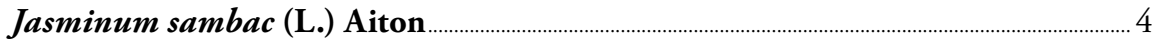

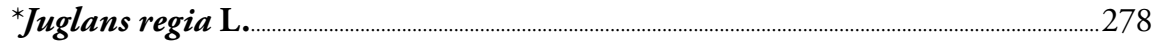

*Juncus effusus L. $\$$

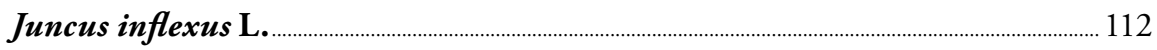

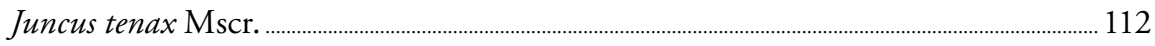

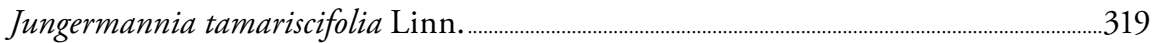


Juniperus oxycedrus Linn.

Juniperus cedrus Webb \& Berthel. subsp. maderensis Rivas Mart. et al. ...........293

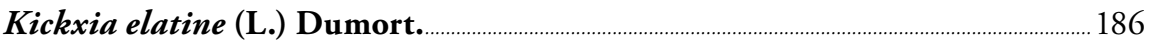

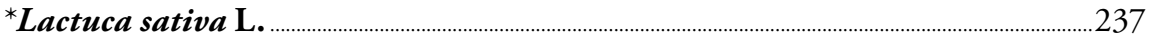

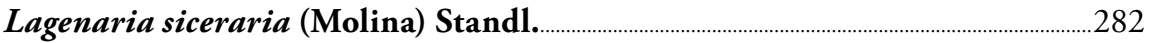

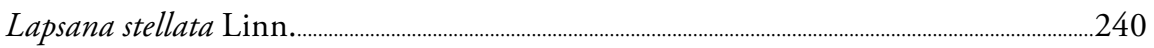

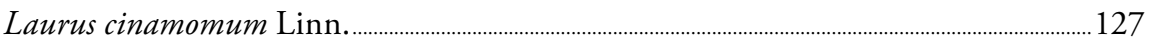

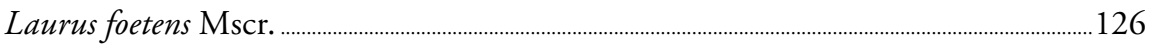

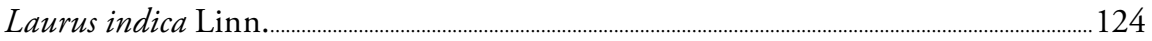

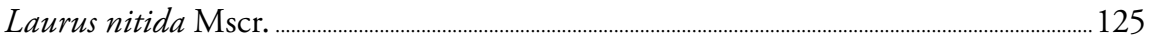

Laurus nobilis Linn....................................................................................................................................... 123

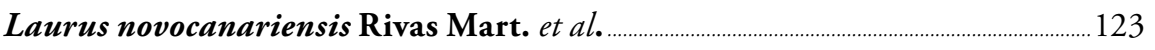

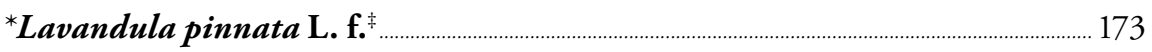

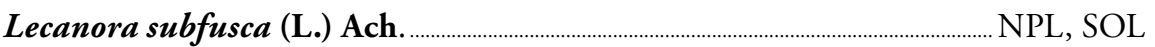

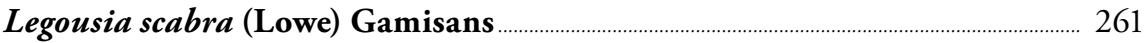

*Lemna minor L. .............................................................................................................................................. 268

Leontodon nudicaulis Mss............................................................................................................2

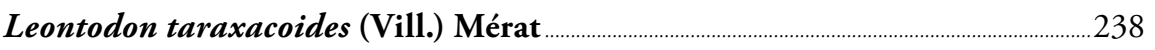

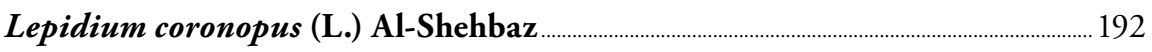

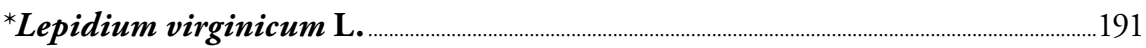

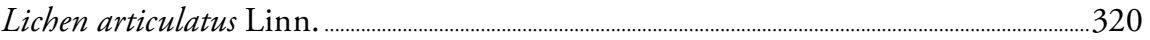

Lichen calicaris Linn..................................................................................................................... 321, 325

Lichen digitatus Linn................................................................................................................................... 322

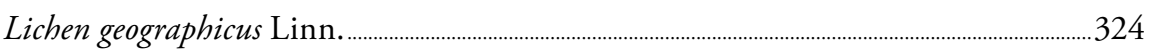

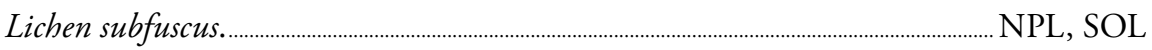

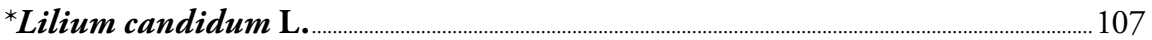

*Linum bienne Mill. ........................................................................................................................................... 102

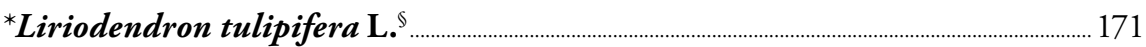

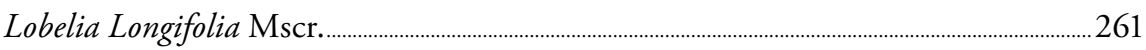

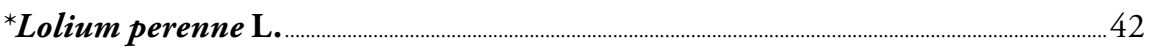

${ }^{*}$ Lolium temulentum L. ...................................................................................................................... 43

${ }^{*}$ Lonicera caprifolium L...........................................................................................................................70

*Lotus corniculatus L............................................................................................................. NPL, SOL

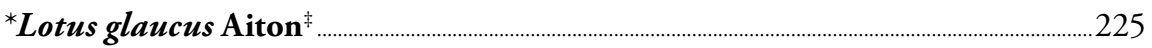

Lotus lancerottensis Webb et Berthel.................................................................... NPL, SOL

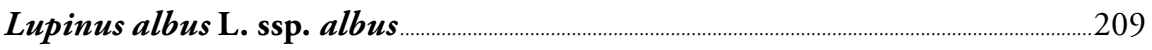

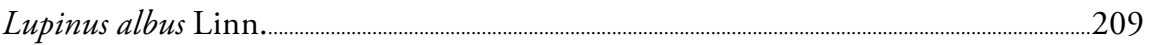




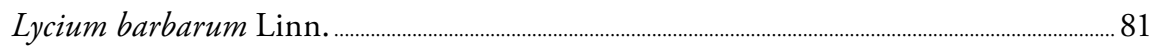

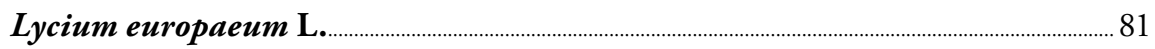

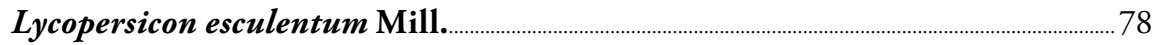

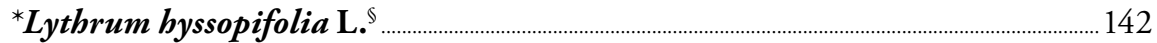

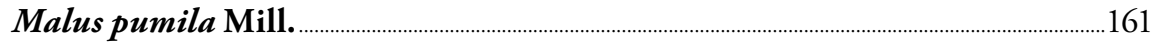

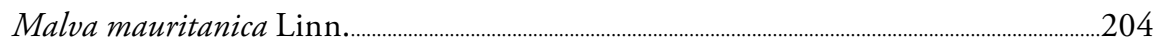

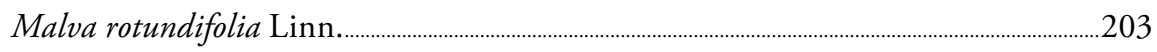

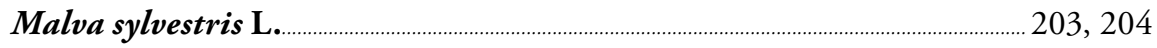

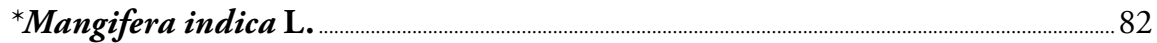

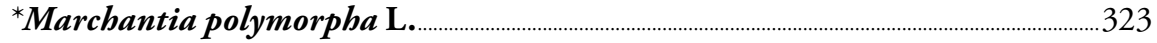

Matricaria parthenium Linn........................................................................................................................ 255

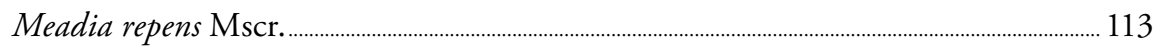

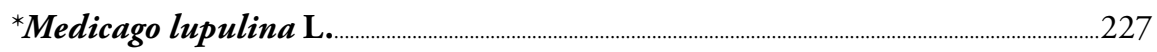

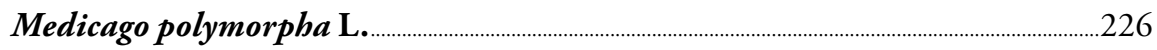

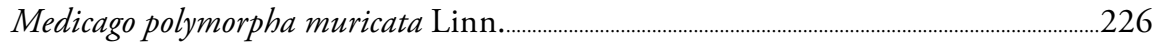

Melilotus angustifolium Linn. .......................................................................................................................22

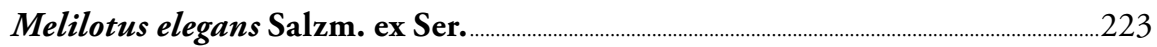

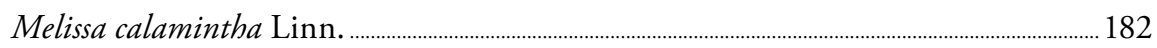

Mentha maderensis insulae, minus odorata Plukn. Alm. 248 [...] ......................NPL, SOL"

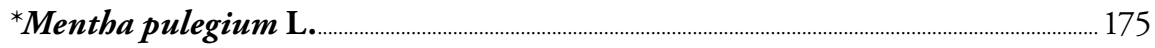

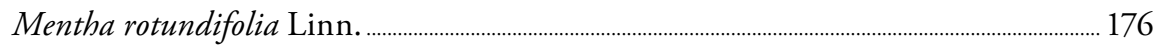

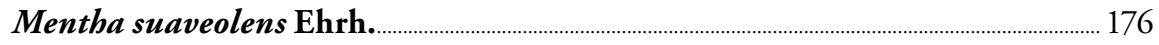

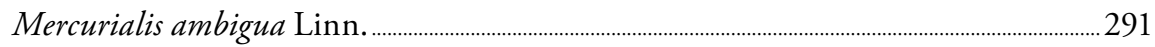

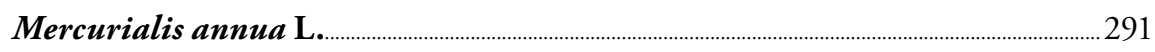

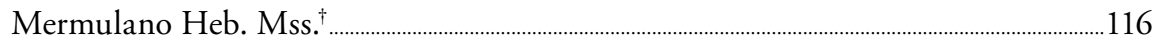

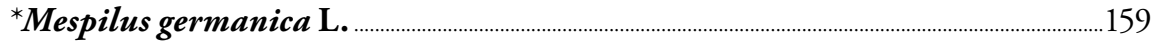

Milium paradoxum Linn....................................................................................................................................... 24

Mimosa Farnesiana Linn. ................................................................................................................. 302

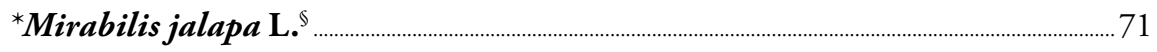

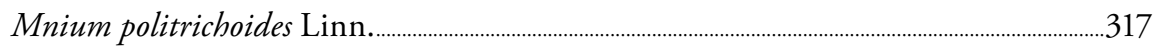

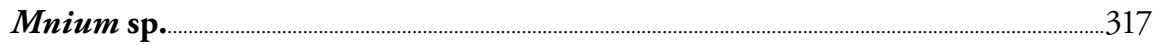

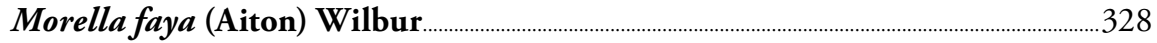

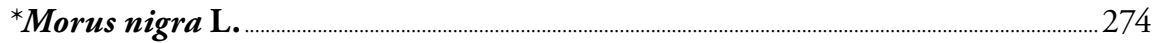

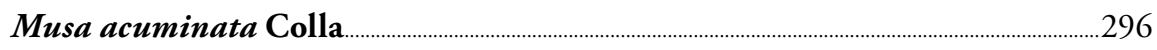

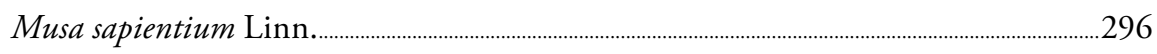

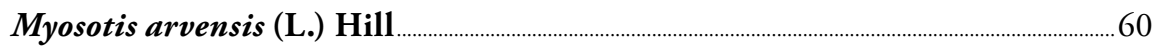

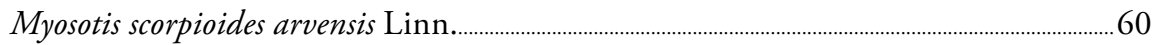




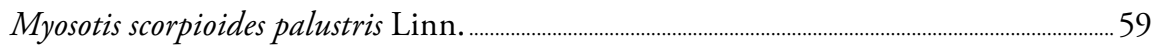

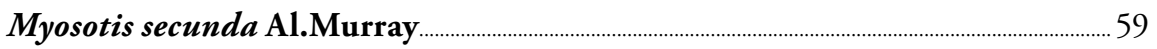

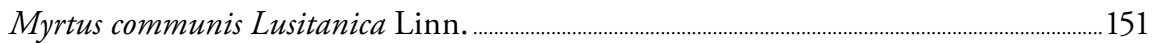

Myrtus communis L. subsp. communis ………………........................................................... 151

Myrtus communis L. subsp. tarentina (L.) Nyman ……………….................................... 152

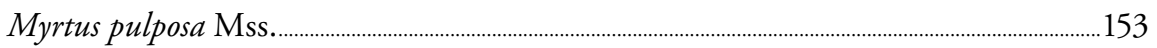

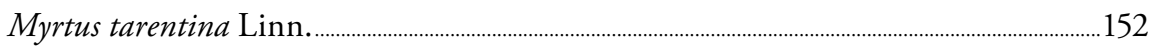

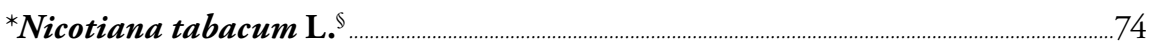

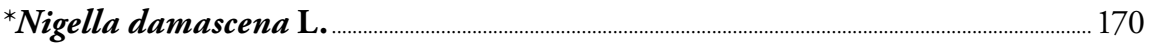

Nyctanthes sambac Linn............................................................................................................................ 4

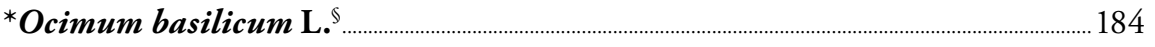

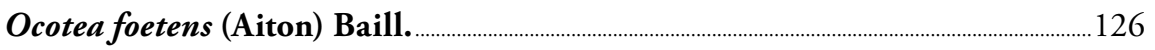

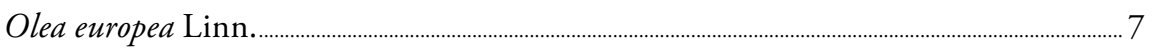

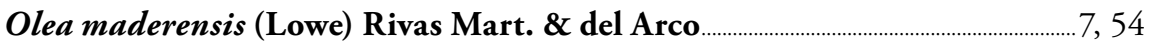

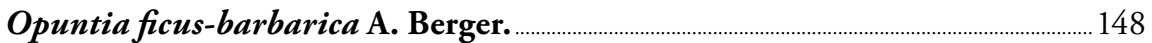

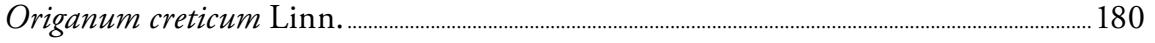

Origanum vulgare L. subsp. virens (Hoffmanns. \& Link) Ietsw. ............................ 180

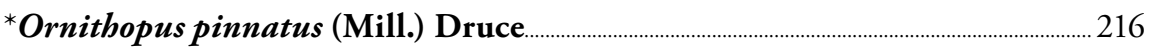

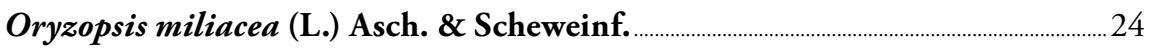

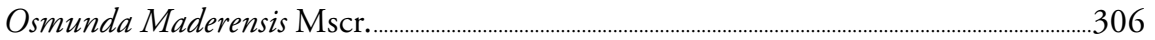

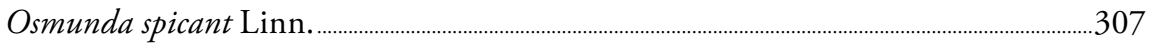

${ }^{*}$ Oxalis corniculata L.......................................................................................................................... 138

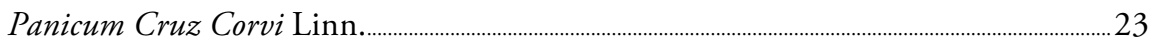

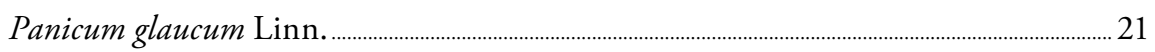

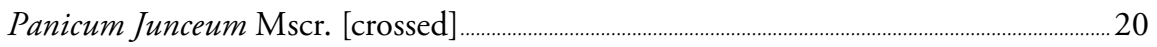

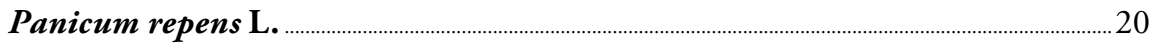

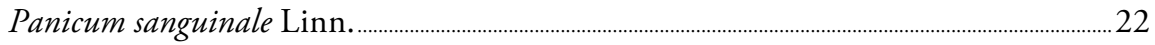

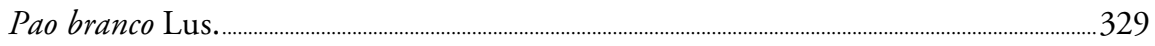

Parietaria judaica L..................................................................................................................... NA, 301

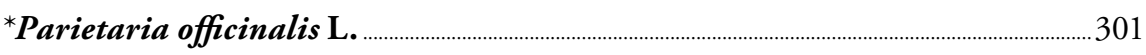

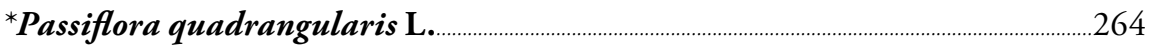

Pennisetum glaucum (L.) R.Br. ......................................................................................................... 21

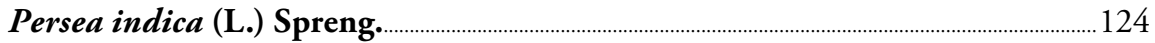

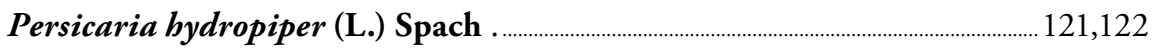

Petrorhagia nanteuilli (Burnat)P.W. Ball \& Heywood ………........................................... 131

Petroselinum crispum (Mill.) Fuss ……………………………………………………..................... 98

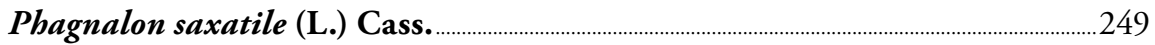




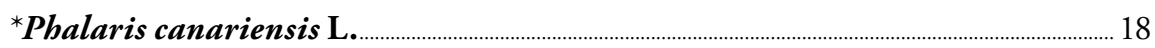

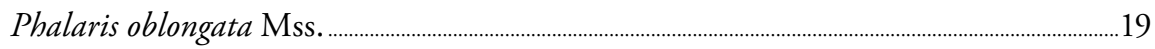

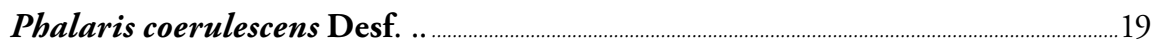

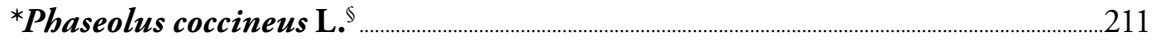

*Phaseolus vulgaris L. $\$$.............................................................................................................................. 210

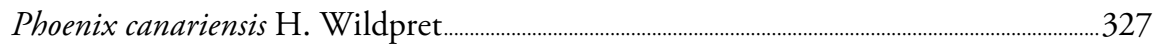

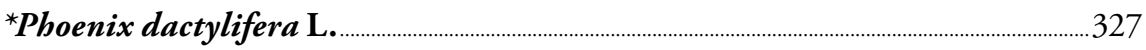

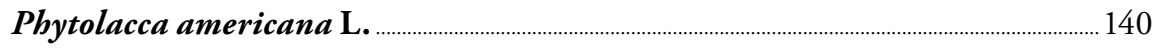

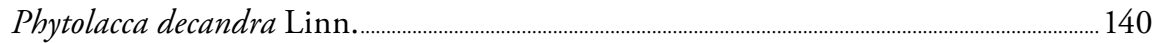

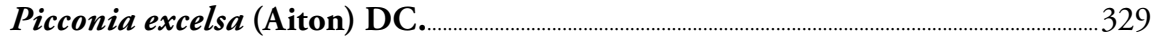

Picris Echioides Linn......................................................................................................................................... 235

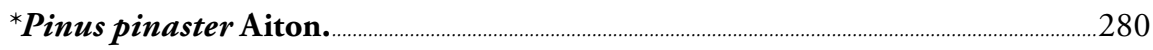

*Pisum sativum L. ............................................................................................................. 212

Plantago albicans $\mathbf{L}$. ..................................................................................................................................... 53

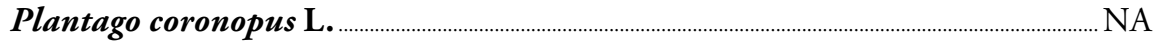

Plantago lagopus L. ...................................................................................................................... NA, 53

*Plantago lanceolata L. ....................................................................................................................51, 53

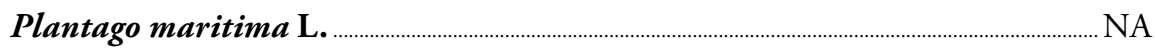

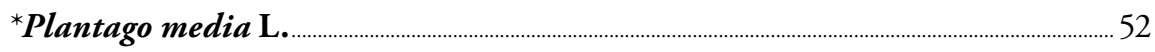

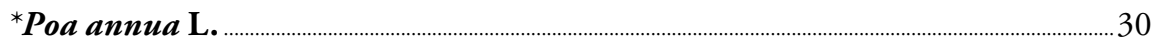

*Polycarpon tetraphyllum (L.) L. .......................................................................................................4 46

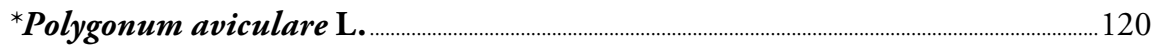

Polygonum Barbatum Linn........................................................................................................................... 122

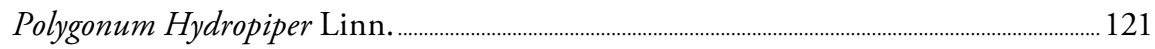

Polypodium fragile Linn. .............................................................................................................................314

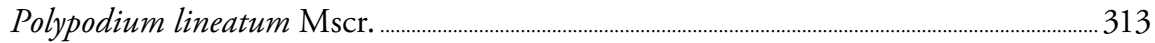

Polypodium Thelypteris Mscr. .................................................................................................................. 312

Polypogon viridis (Gouan) Breist. ..........................................................................................................29

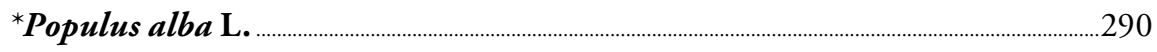

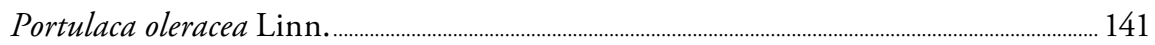

Portulaca oleracea L. subsp. oleracea ………………………………………………………..... 141

*Potentilla reptans L............................................................................................................................................ 167

Poterium sanguisorba Linn................................................................................................................ 277

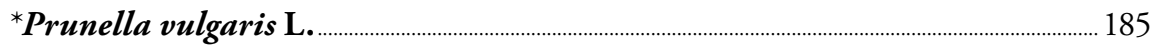

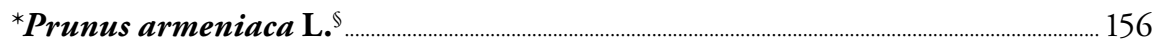

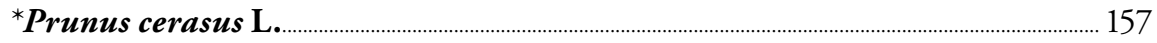

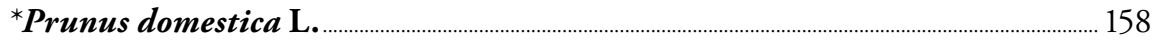




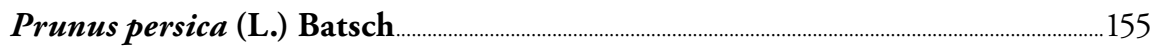

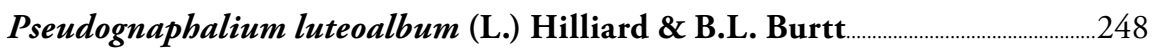

Psidium guajava L. .......................................................................................................................... 149

Psidium pyriferum Linn............................................................................................................................. 149

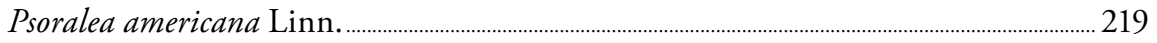

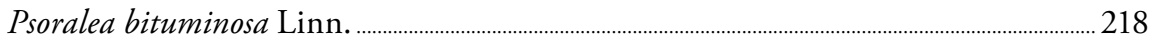

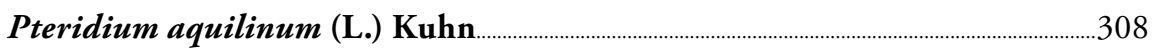

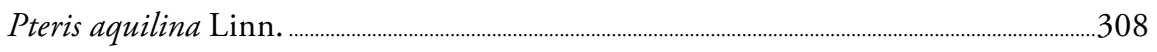

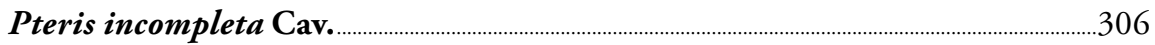

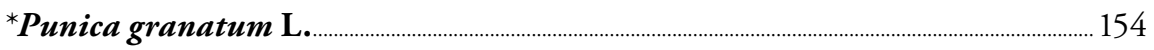

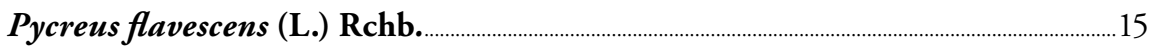

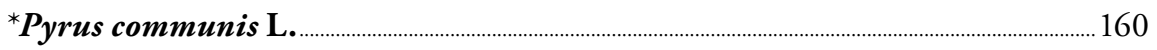

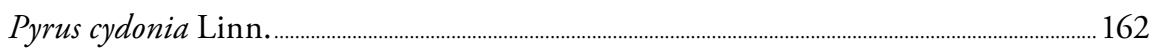

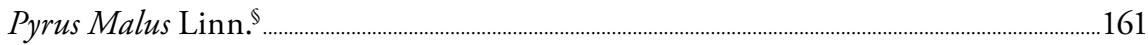

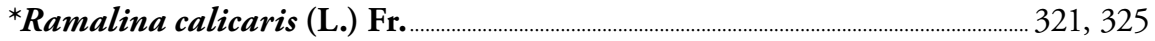

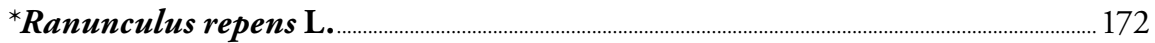

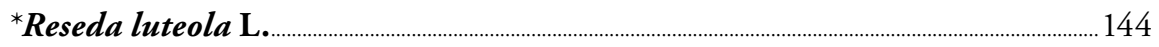

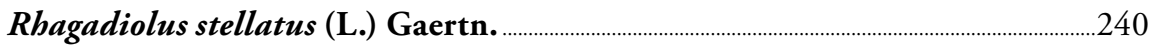

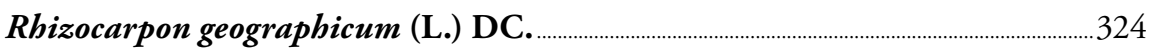

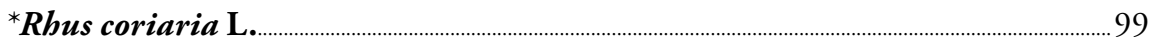

${ }^{*}$ Ribes grossularia L. ........................................................................................................................................ 84

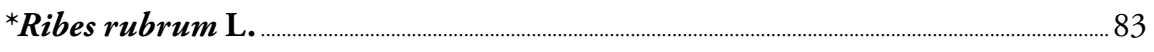

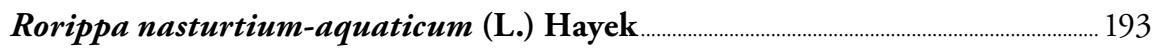

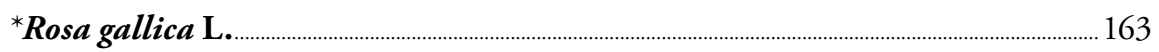

${ }^{*}$ Rosmarinus officinalis L....................................................................................................................... 10

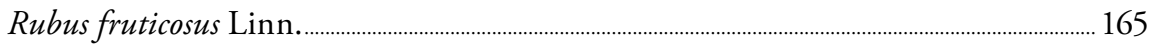

Rubus pedatus Mss............................................................................................................................................ 164

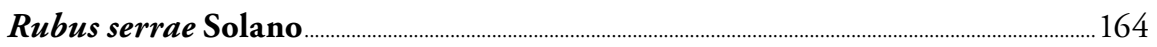

Rubus ulmifolius Schott ............................................................................................................................. 165

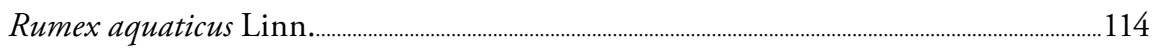

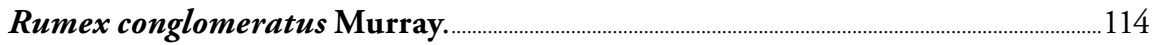

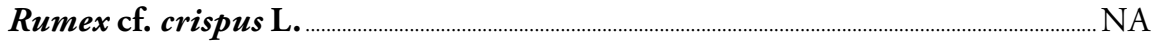

Rumex maderensis Lowe

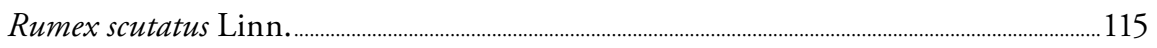

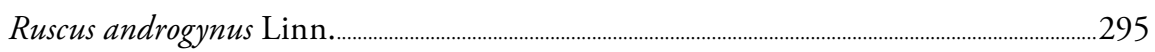

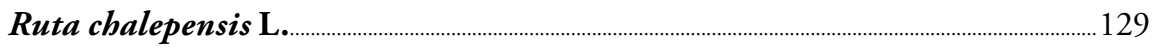

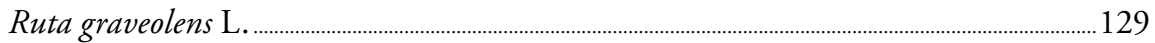




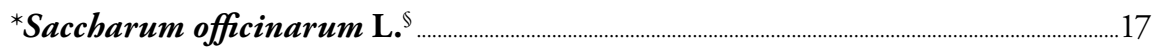

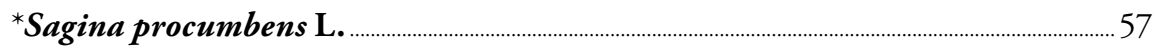

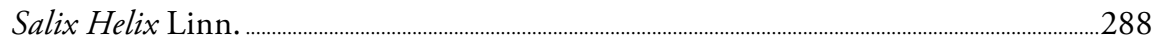

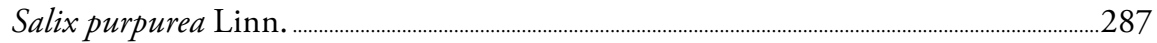

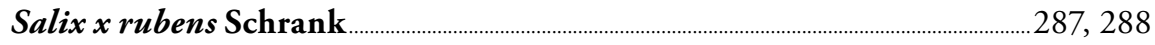

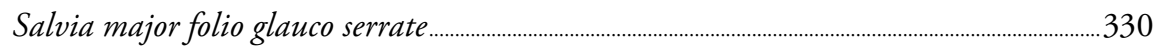

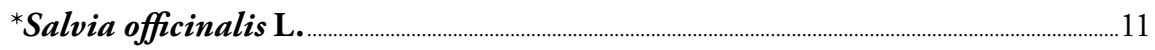

${ }^{*}$ Sambucus ebulus L. ................................................................................................................................. 100

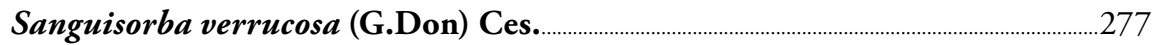

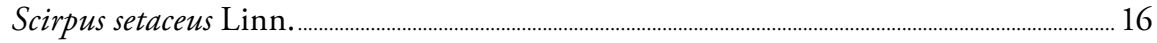

Scolymus hispanicus Linn............................................................................................................................ 242

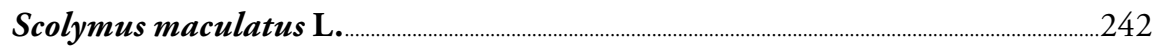

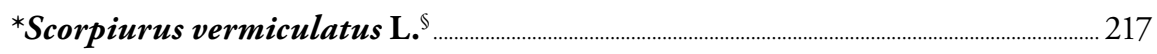

*Scrophularia scorodonia L. ........................................................................................................ 187

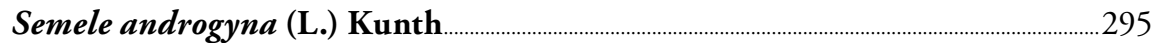

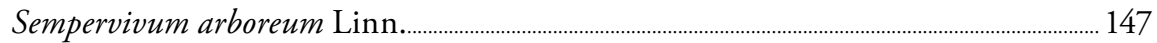

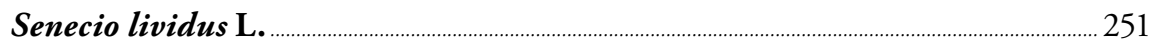

Senecio viscosus Linn............................................................................................................................... 251

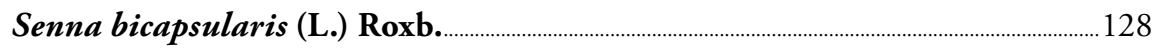

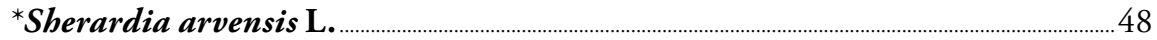

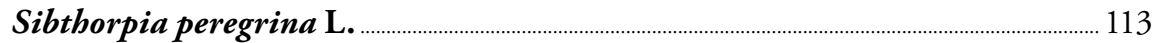

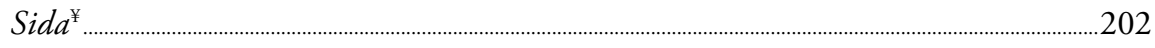

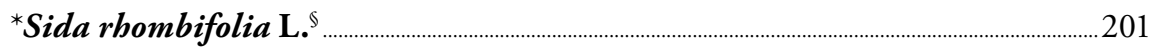

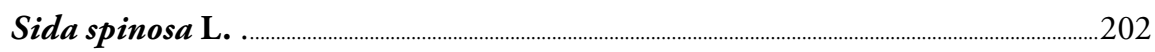

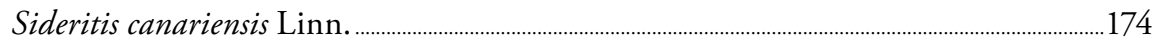

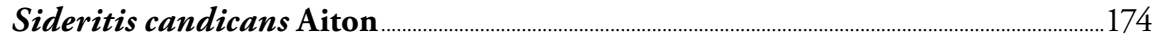

Sideroxylon mirmulans R.Br. ........................................................................................................... 116

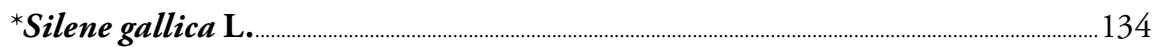

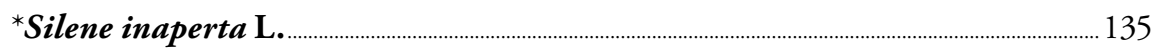

Silene vulgaris (Moench) Garcke subsp. vulgaris ................................................................ 133

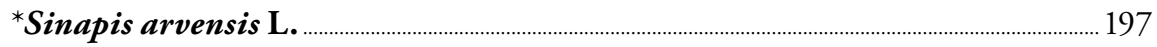

Sisymbrium Nasturtium Linn................................................................................................................... 193

Sisymbrium officinale (L.) Scop.

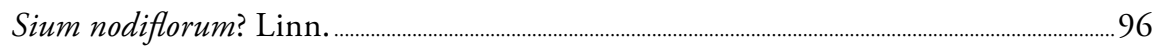

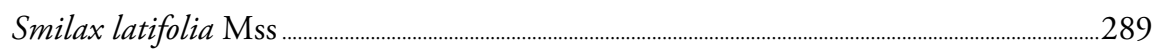

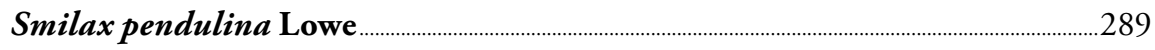

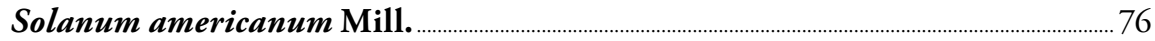




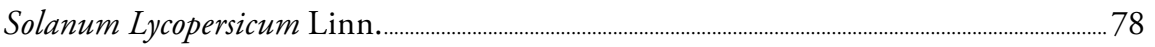

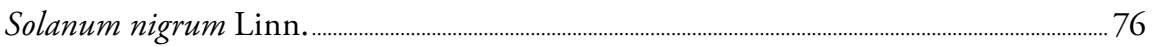

*Solanum pseudocapsicum L. ............................................................................................................ 77

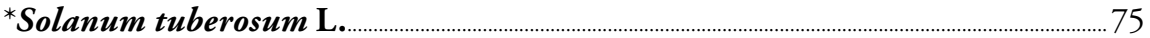

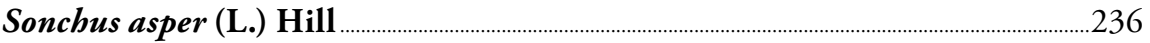

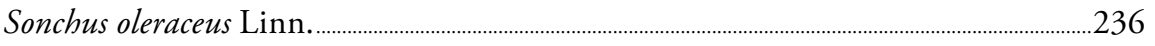

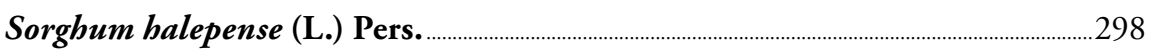

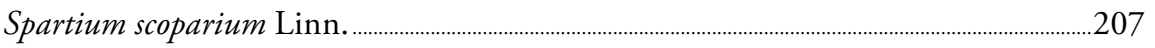

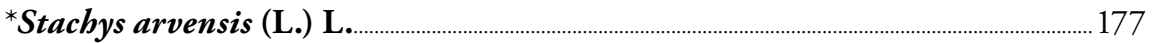

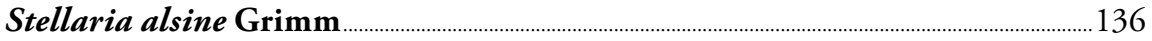

Stellaria graminea Linn................................................................................................................................. 136

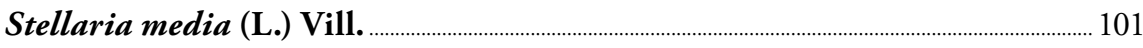

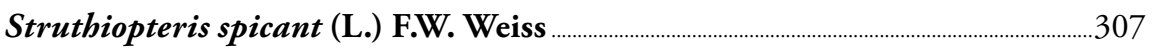

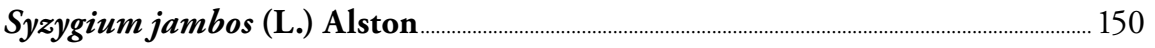

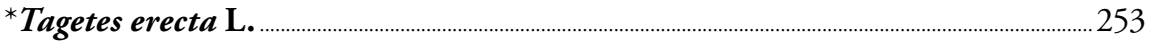

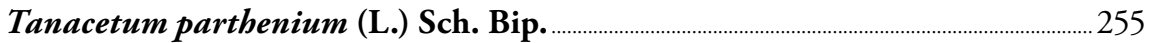

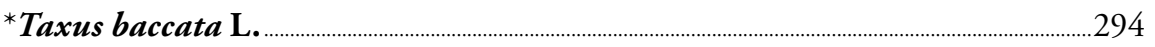

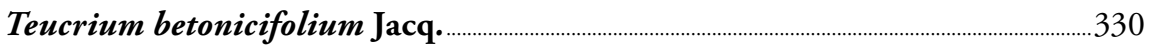

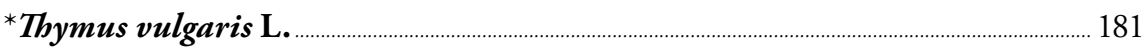

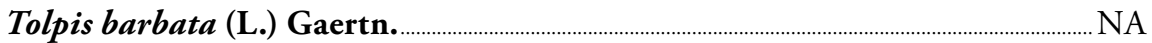

Tolpis succulenta (Aiton) Lowe …..............................................................................................239

Torilis arvensis (Huds.) Link subsp. neglecta (Spreng.) Thell. ....................................... 93

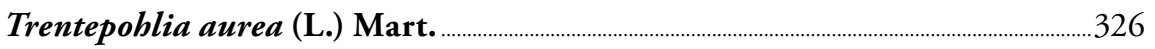

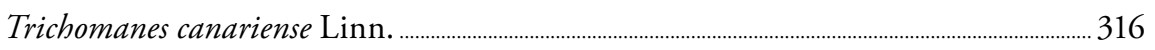

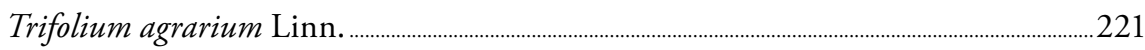

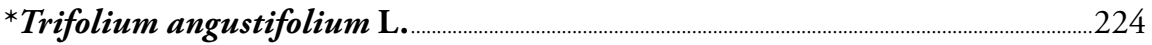

Trifolium dubium Sibth............................................................................................................................... 221

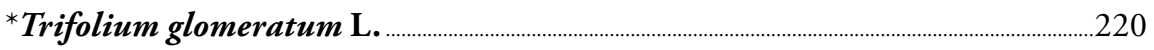

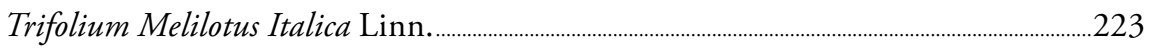

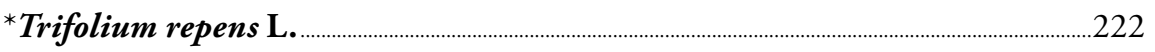

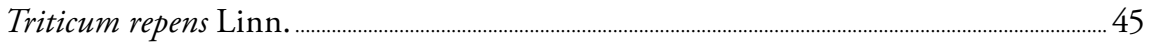

Tropaeolum minus L............................................................................................................................................

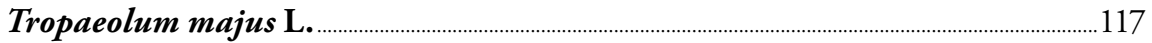

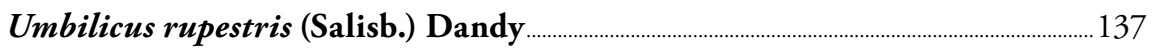

Urtica membranacea Savigny ………………………………….......................................................2. 273

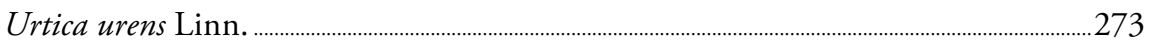

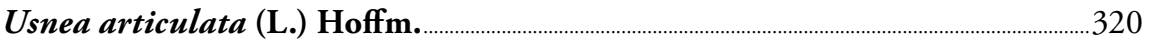


Vaccinium elevatum Mss...

Vaccinium padifolium Sm.

*Verbena officinalis L.

Veronica anagallis Linn. 8

Veronica anagallis-aquatica $\mathrm{L}$. 8

Vicia gracilis Mss. 213

Vicia hirsuta (L.) Gray... 214

Vicia articulata Hornm.

*Viola odorata L. 262

*Vitis vinifera L.. 86

Vulpia bromoides (L.) Gray. 26

*Zea mays $\mathrm{L}$. 269

Zingiber officinale Roscoe. 2

Notes to the Taxonomic Index:

* Taxon for which the accepted name matches that written in Banks's journal list or Solander's flora.

$\$$ Name reported in Banks's journal list either (i) is a slight orthographical variant of the accepted scientific name (ii) has the specific epithet in upper case or (iii) has an authority does not match that of the accepted scientific name.

¥ Banks’s journal list only reports the generic name.

‡ Name reported in Banks’s journal list with the codes “Mscr." or "Mss.” These names were considered new taxa by Banks and Solander.

$\dagger$ Refers to Thomas Heberden.

Species for which there is herbarium material.

NA refers to species for which there is herbarium material but it was not recorded in Banks's journal list nor in Solander's unpublished Primitiae Florae Maderensis, sive catalogus Plantarum in Insula Madera.

NPL refers to species listed in the index to the contents of the drying books but not recorded in Banks's journal list. nal list.

SOL refers to species listed in Solander's flora but not recorded in Banks's jour- 